University of Tennessee Health Science Center

UTHSC Digital Commons

\title{
A Passive Pure Moment Protocol for Testing Spine Segments: Development and Application
}

Kyle David Fraysur

University of Tennessee Health Science Center

Follow this and additional works at: https://dc.uthsc.edu/dissertations

Part of the Investigative Techniques Commons, and the Medical Biotechnology Commons

\section{Recommended Citation}

Fraysur, Kyle David, "A Passive Pure Moment Protocol for Testing Spine Segments: Development and Application" (2010). Theses and Dissertations (ETD). Paper 86. http://dx.doi.org/10.21007/ etd.cghs.2010.0098.

This Thesis is brought to you for free and open access by the College of Graduate Health Sciences at UTHSC Digital Commons. It has been accepted for inclusion in Theses and Dissertations (ETD) by an authorized administrator of UTHSC Digital Commons. For more information, please contact jwelch30@uthsc.edu. 


\title{
A Passive Pure Moment Protocol for Testing Spine Segments: Development and Application
}

\begin{abstract}
The pure moment protocol is the accepted standard for performing in-vitro biomechanical testing of spinal devices. Published studies predominantly report range of motion and flexibility data, but information regarding the segment center of rotation is also relevant. Most current pure moment platforms are not sensitive enough to accurately calculate the instantaneous axis of rotation (IAR) for a segment throughout a bending motion. The purpose of this study was to simulate a pure moment protocol using a programmable spine robot, and use the data gathered to calculate the IAR for harvested specimen and those implanted with a constrained total disc replacement (TDR) device.
\end{abstract}

Six human lumbar single-level motion segment units (MSUs) at the L4-L5 level were dissected and potted. The average age of the spines was $47 \pm 11.4$ years. The robot was programmed to rotate the specimen in flexion and extension and left and right lateral bending in 0.25 degree increments, minimizing shear and axial loading after each rotation, thereby finding a quasistatic rotational path of minimal loading. The specimens were rotated to $8 \mathrm{Nm}$ of sagittal moment during flexion and extension and $6 \mathrm{Nm}$ of lateral moment during lateral bending. During lateral testing, the specimens were unconstrained axially. Once harvested testing was completed, specimens were implanted with a constrained ProDisc-L implant (Synthes Inc., West Chester, PA) by a spine surgeon under fluoroscopy. All pure moment testing was repeated on the implanted specimen.

Throughout testing, the specimens underwent an average off-axis force of $1.51 \mathrm{~N}$. With an average perpendicular distance of $0.062 \mathrm{~mm}$, this force value contributed $0.000094 \mathrm{Nm}$ to the maximum bending moment, meaning the test platform was $99.99 \%$ free of off-axis loading. During flexion and extension tests the specimens rotated an average of $9.90 \pm 2.23$ degrees and $3.40 \pm 1.43$ degrees respectively. During left and right lateral bending tests the specimens rotated an average of $6.21 \pm 1.34$ degrees and $5.64 \pm 1.77$ degrees respectively. These values are in agreement with other published studies of lumbar spinal biomechanics. Range of motion comparisons between the harvested and implanted specimen showed a significant difference in right lateral bending and combined lateral bending (one-way repeated measures ANOVA with SNK test, $p<0.05$ ). No significant differences were observed for flexion or extension motions.

IAR values were calculated for the harvested and implanted specimen for flexion and extension testing and normalized based on the height and anterior-to-posterior (A-P) width of the disc. These values were compared with a One-Way ANOVA with Dunn's comparison test between locations of $X$ and $Y$ coordinates for each IAR within and between conditions $(p<0.05)$. All comparisons save for the position of $Y$ coordinates in harvested testing between flexion and extension showed significance.

Future work will be to allow for a user-inputted axial load to simulate a net muscle vector, use of the protocol with other constrained as well as unconstrained TDR devices, and use of the protocol within multi-body studies.

\section{Document Type}

Thesis

\section{Degree Name}

Master of Science (MS) 


\section{Program}

Biomedical Engineering

Research Advisor

Denis J. DiAngelo, Ph.D.

\section{Keywords}

center of rotation, instantaneous axis of rotation, load control, lumbar spine, pure moment, spine biomechanics

\section{Subject Categories}

Analytical, Diagnostic and Therapeutic Techniques and Equipment | Investigative Techniques | Medical Biotechnology | Medicine and Health Sciences 


\title{
A PASSIVE PURE MOMENT PROTOCOL FOR TESTING SPINE SEGMENTS: DEVELOPMENT AND APPLICATION
}

\author{
A Thesis \\ Presented for \\ The Graduate Studies Council \\ The University of Tennessee \\ Health Science Center
}

\author{
In Partial Fulfillment \\ Of the Requirements for the Degree \\ Master of Science \\ In the Joint Graduate Program in Biomedical Engineering and Imaging \\ From The University of Tennessee \\ and \\ The University of Memphis
}

By

Kyle David Fraysur

May 2010 
Copyright (C) 2008 by Kyle David Fraysur All rights reserved. 


\section{Dedication}

This thesis is dedicated to my beautiful wife

Elizabeth Nicole Fraysur

who was incredibly positive and supportive throughout this entire process and to my son

Greyson David Fraysur

who never ceases to amaze. 


\section{Acknowledgements}

I would like to thank everyone that helped make my graduate experience enjoyable and productive. First, I would like to thank my advisor, Dr. Denis DiAngelo, for his guidance and patience. I would like to thank my committee members, Dr. Brian Kelly and Dr. Gladius Lewis for their input on this research. Elizabeth Sander and Nephi Zufelt offered excellent guidance to a young student entering an often hectic lab, and for that they deserve special thanks. Braham Dhillon, Karen Sedacki, and Dan Wido also deserve special thanks for all their help with all the projects I worked on while in the lab. I want to thank all the members of the Joint Implant and Biomechanics Lab.

Finally, I must thank my amazing wife, Elizabeth, for her incredible patience and support throughout my collegiate experience. None of this would have been possible without her. 


\begin{abstract}
The pure moment protocol is the accepted standard for performing in-vitro biomechanical testing of spinal devices. Published studies predominantly report range of motion and flexibility data, but information regarding the segment center of rotation is also relevant. Most current pure moment platforms are not sensitive enough to accurately calculate the instantaneous axis of rotation (IAR) for a segment throughout a bending motion. The purpose of this study was to simulate a pure moment protocol using a programmable spine robot, and use the data gathered to calculate the IAR for harvested specimen and those implanted with a constrained total disc replacement (TDR) device.

Six human lumbar single-level motion segment units (MSUs) at the L4-L5 level were dissected and potted. The average age of the spines was $47 \pm 11.4$ years. The robot was programmed to rotate the specimen in flexion and extension and left and right lateral bending in 0.25 degree increments, minimizing shear and axial loading after each rotation, thereby finding a quasistatic rotational path of minimal loading. The specimens were rotated to $8 \mathrm{Nm}$ of sagittal moment during flexion and extension and $6 \mathrm{Nm}$ of lateral moment during lateral bending. During lateral testing, the specimens were unconstrained axially. Once harvested testing was completed, specimens were implanted with a constrained ProDisc-L implant (Synthes Inc., West Chester, PA) by a spine surgeon under fluoroscopy. All pure moment testing was repeated on the implanted specimen.
\end{abstract}

Throughout testing, the specimens underwent an average off-axis force of $1.51 \mathrm{~N}$. With an average perpendicular distance of $0.062 \mathrm{~mm}$, this force value contributed $0.000094 \mathrm{Nm}$ to the maximum bending moment, meaning the test platform was $99.99 \%$ free of off-axis loading. During flexion and extension tests the specimens rotated an average of $9.90 \pm 2.23$ degrees and $3.40 \pm 1.43$ degrees respectively. During left and right lateral bending tests the specimens rotated an average of $6.21 \pm 1.34$ degrees and $5.64 \pm 1.77$ degrees respectively. These values are in agreement with other published studies of lumbar spinal biomechanics. Range of motion comparisons between the harvested and implanted specimen showed a significant difference in right lateral bending and combined lateral bending (one-way repeated measures ANOVA with SNK test, $\mathrm{p}<0.05$ ). No significant differences were observed for flexion or extension motions.

IAR values were calculated for the harvested and implanted specimen for flexion and extension testing and normalized based on the height and anterior-to-posterior (A-P) width of the disc. These values were compared with a One-way ANOVA with Dunn's comparison test between locations of $\mathrm{X}$ and $\mathrm{Y}$ coordinates for each IAR within and between conditions $(\mathrm{p}<0.05)$. All comparisons save for the position of $Y$-coordinates in harvested testing between flexion and extension showed significance.

Future work will be to allow for a user-inputted axial load to simulate a net muscle vector, use of the protocol with other constrained as well as unconstrained TDR devices, and use of the protocol within multi-body studies. 


\section{Table of Contents}

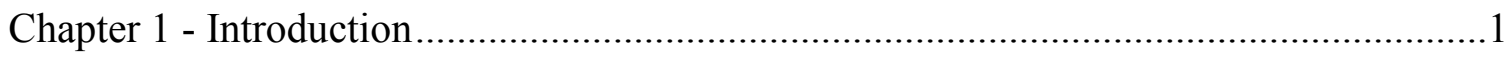

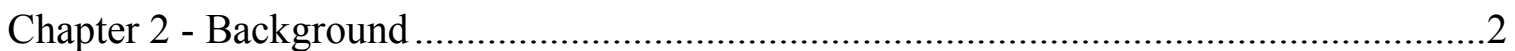

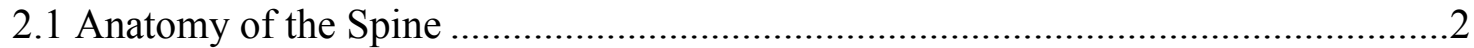

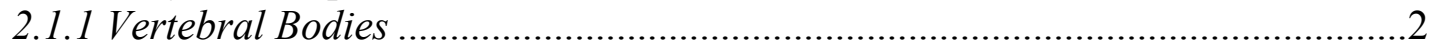

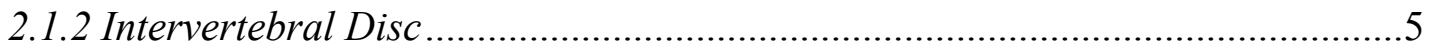

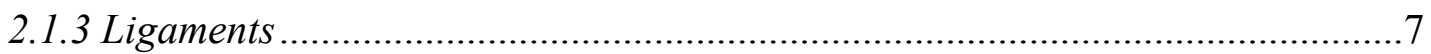

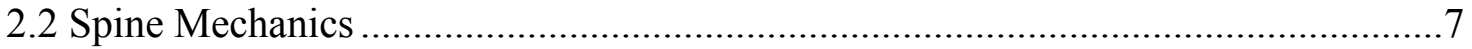

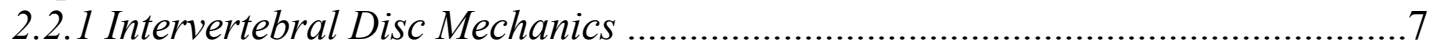

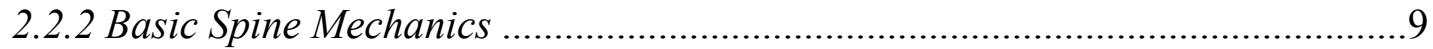

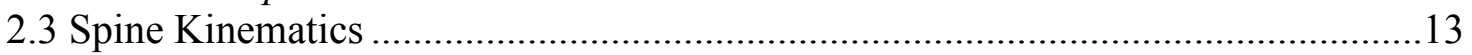

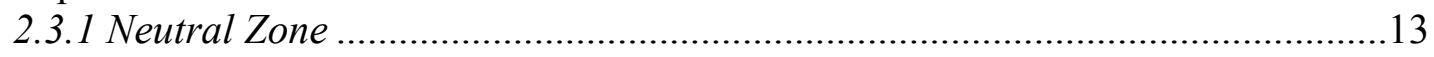

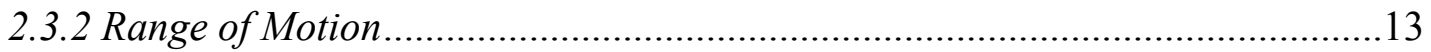

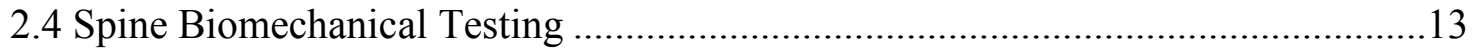

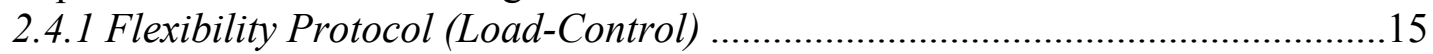

2.4.2 Stiffness Protocol (Displacement-Control)......................................................17

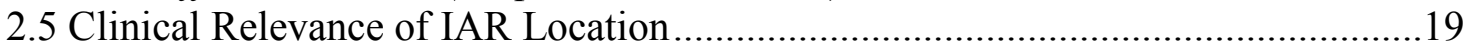

Chapter 3 - Creation of a Pure Moment Protocol for Studying Instantaneous Axis of

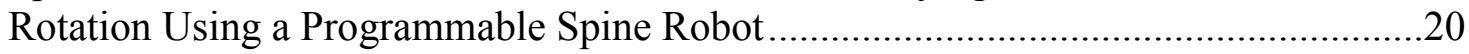

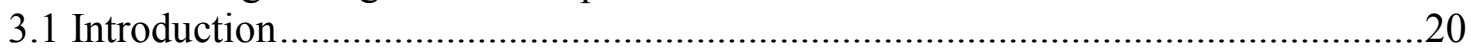

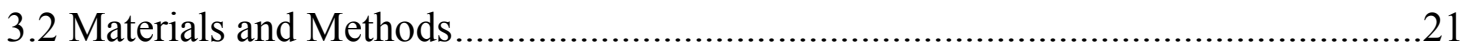

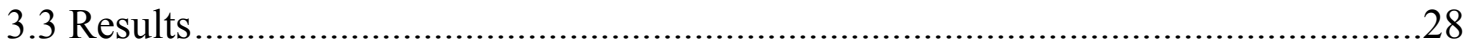

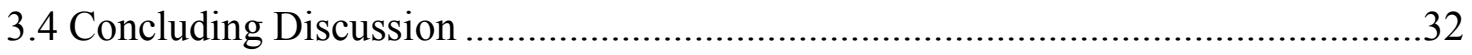

Chapter 4 - An In-vitro Comparison of Harvested Lumbar Segments to Those Implanted with a Constrained Total Disc Replacement Device under Pure Moment

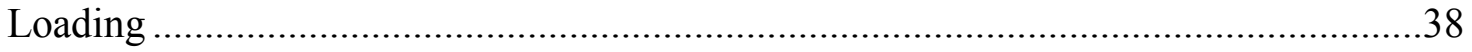

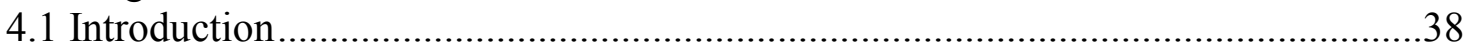

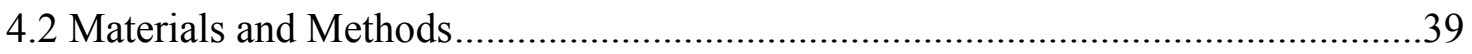

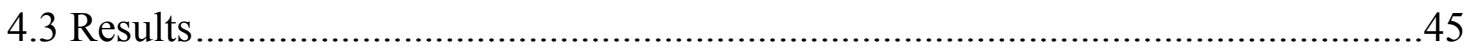

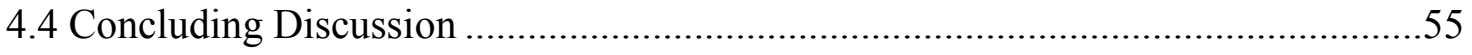

Chapter 5 - Conclusions and Limitations....................................................................56

Chapter 6 - Recommendations for Future Work............................................................58

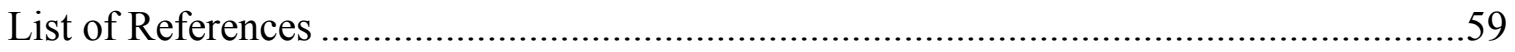






\section{List of Tables}

Table 3-1 Spine Robot Specifications................................................................ 25

Table 3-2 Rotation (Degrees) during Four Modes of Bending................................... 29

Table 3-3 Average Forces (N) along the Harvested Quasistatic Path .......................... 30

Table 3-4 Off-axis Loading in Current Study Compared to Common Pure Moment

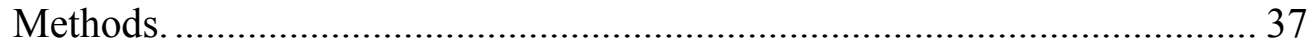

Table 4-1 Average Forces (N) along the Quasistatic Path.......................................... 48

Table 4-2 Significant Differences among IAR Locations ......................................... 54 


\section{List of Figures}

Figure 2-1 The Full Human Spinal Column ........................................................... 3

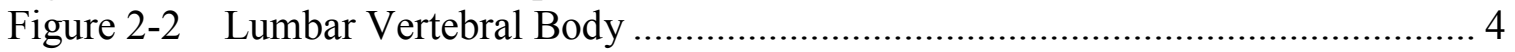

Figure 2-3 The Three Components of the Intervertebral Disc................................... 6

Figure 2-4 Ligaments of the Lumbar Spine ............................................................. 8

Figure 2-5 Disc Converting Axial to Radial Force ..................................................... 10

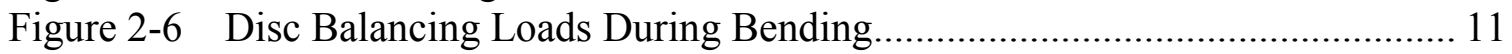

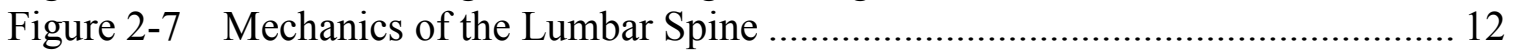

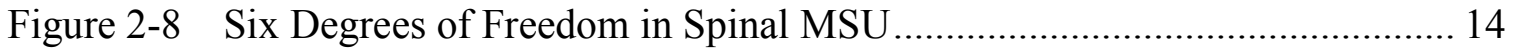

Figure 2-9 Neutral and Elastic Zones of Spinal Motion .............................................. 14

Figure 2-10 Illustration of Common Pure Moment Setup ............................................ 16

Figure 2-11 Illustration of Eccentric Load Protocol Setup ........................................... 18

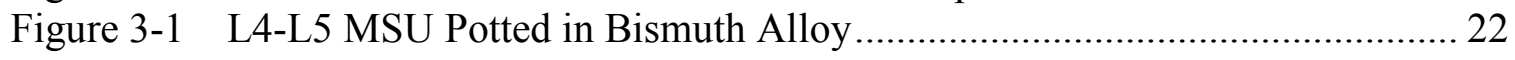

Figure 3-2 Spine Robot with Gimbal Attachment ................................................... 23

Figure 3-3 Flowchart Outlining Pure Moment Testing Protocol................................ 26

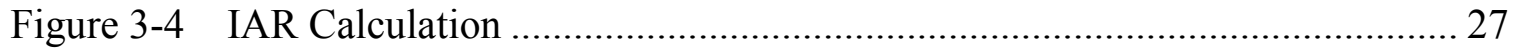

Figure 3-5 Control Specimen Graph....................................................................... 29

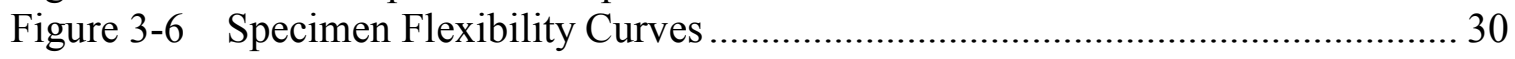

Figure 3-7 Shear Forces for Each Specimen during Four Modes of Bending ............... 31

Figure 3-8 Axial Forces for Each Specimen during Four Modes of Bending .............. 31

Figure 3-9 Calculated IAR Locations on Radiographs .......................................... 33

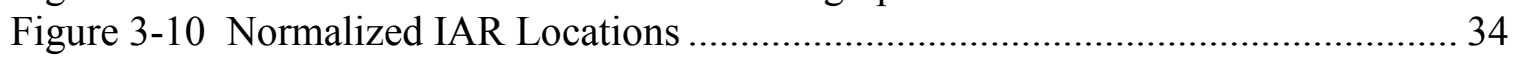

Figure 3-11 Comparison to Published In-vitro Rotations ........................................... 35

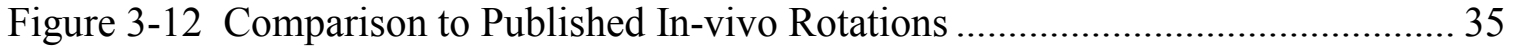

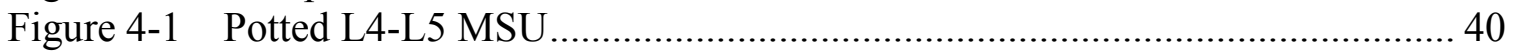

Figure 4-2 Assembled ProDisc-L (Synthes Spine) Total Disc Arthroplasty Device.... 40

Figure 4-3 ProDisc-L Implantation....................................................................... 42

Figure 4-4 Lumbar MSU before and after ProDisc-L Implantation ............................ 42

Figure 4-5 Spine Robot with Labeled Degrees of Freedom .................................... 43

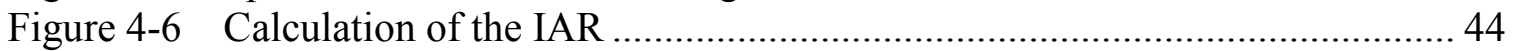

Figure 4-7 Harvested Flexibility Curves................................................................... 46

Figure 4-8 Implanted Flexibility Curves............................................................... 46

Figure 4-9 Comparing Ranges of Motion between Conditions ................................... 47

Figure 4-10 Comparing Total Ranges of Motion between Conditions........................... 47

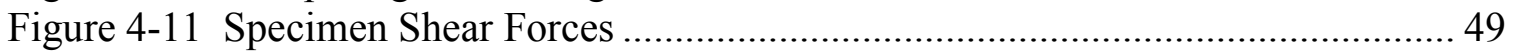

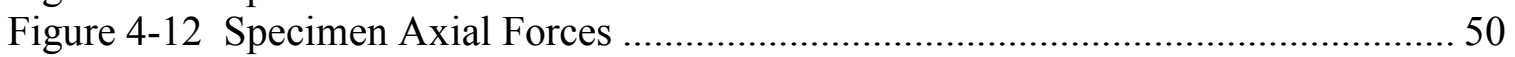

Figure 4-13 Calculated Harvested IAR Locations ........................................................ 51

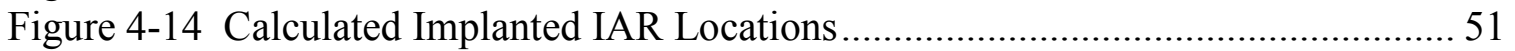

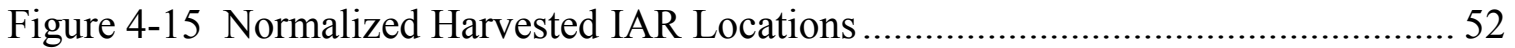

Figure 4-16 Normalized Implanted IAR Locations ................................................. 53 


\section{List of Abbreviations}

$\begin{array}{ll}\text { A-P } & \text { Anterior-posterior } \\ \text { ALE } & \text { Adjacent level effect } \\ \text { ANOVA } & \text { Analysis of variance } \\ \text { COR } & \text { Center of rotation } \\ \text { DDD } & \text { Degenerative disc disease } \\ \text { IAR } & \text { Instantaneous axis of rotation } \\ \text { MSU } & \text { Motion segment unit } \\ \text { TDR } & \text { Total disc replacement }\end{array}$




\section{Chapter 1 - Introduction}

As the mean age of the American population continues to increase, the number of patients diagnosed with degenerative disc disease is continuing to grow. For patients for whom medicinal and physical therapy treatments have failed, the standard surgical treatment has been spinal fusion. Conventional biomechanical testing has been developed to study the effects of fusion on the spine and to attempt to replicate in-vivo spinal motions in-vitro. As more complex devices and techniques have been developed, the need to advance biomechanical testing has grown. ${ }^{42,60}$

Pure moment testing is a popular methodology because it produces loading conditions that theoretically should be equal across all spinal levels that are tested, yielding repeatable results easily comparable between labs. Additionally, the pure moment test protocols are reproducible on relatively simple testing platforms, making this testing type accessible for all labs. Unfortunately, because such platforms lack the mechanical components to perform more advanced testing, pure moment methodologies have not advanced significantly. If a system with a greater range of motion and the ability to accurately control and measure both load and position were utilized for pure moment testing, then advanced testing might be more easily developed. ${ }^{27,42}$

Most load-control spinal studies report data regarding range of motion and flexibility, but very few report data regarding the instantaneous axis of rotation (IAR). The location of the IAR is believed by many researchers to be a clinically significant characteristic of spinal motion that may give indications regarding degenerative disease and trauma. ${ }^{53,54}$ Unfortunately, there is confusion in the literature regarding the positions of the IAR in the lumbar spine due to the testing setups lacking instruments sensitive enough for accurate measurements. ${ }^{12,14}$ Due to this confusion, clinicians are hesitant to use the IAR to make specific diagnoses. A testing platform with very accurate measurement capabilities should allow for accurate calculation of the IAR along the rotational path of the specimen.

The goal of this research was to use the multi-axis programmable Spine Robot at the University of Tennessee Health Science Center to develop a repeatable and highly accurate pure moment testing method for harvested tissue. Once the pure moment protocol was established, testing comparing harvested tissue to that which had been implanted with a total disc arthroplasty device was completed. The range of motions and locations of the instantaneous axes of rotation were compared within the two specimen groups. Two manuscripts that will be submitted for publication constitute the main body of this thesis. 


\section{Chapter 2 - Background}

This chapter is divided into five sections. Section one discusses the anatomical structures of the lumbar spine. The second section is about the basic mechanics of the lumbar spine motion segment units. Section three gives an overview of the kinematic parameters of the lumbar motion segment units. The fourth section discusses the two types of spinal biomechanics tests, common protocols that use the two approaches, and advantages and drawbacks to each approach. Finally, the fifth section is about the importance of the IAR for spinal biomechanics.

\subsection{Anatomy of the Spine}

The human spine consists of 25 vertebral bodies arranged in column fashion at the posterior of the neck and trunk of the body. The spine is broken down into four sections from top to bottom: cervical (seven vertebral bodies), thoracic (12 vertebral bodies), lumbar (five vertebral bodies), and sacrum (five fused sacral vertebrae and three to four fused coccygeal vertebrae). As seen in Figure 2-1, the cervical and lumbar sections have a naturally lordotic curvature while the thoracic and sacral sections have a naturally kyphotic curvature. A motion segment unit (MSU) is a spinal structure consisting of two vertebral bodies, one intervertebral disc, and all ligaments and muscles connecting the unit. $^{64}$

\subsubsection{Vertebral Bodies}

Vertebral bodies are the bony structures of the MSU and contain an inner cancellous structure for vascularity with an outer cortical shell for strength. The cortical shell ranges from 1-3mm in thickness. Vertebral bodies consist of an anterior portion (body) and a posterior portion (foramen) as seen in Figure 2-2. The anterior body contains the attachment sites to the intervertebral disc and has a thin layer of cartilage to provide nutrients to the disc. This function is necessary because the disc is an avascular structure and is accomplished through pores which allow nutrient diffusion.

The posterior portion of the vertebral body contains the pedicle, lamina, transverse processes, spinous process, and zygapophyseal joints. The lamina and pedicle form the enclosure that protects the spinal cord, descending the entire length of the spinal column. The transverse processes protrude laterally and give the attachment sites for muscles and ligaments discussed further in Section 2.1.3. The spinous process is the most posterior structure of the vertebral body and also allows for attachments of muscle and ligaments.

The zygapophyseal joints (better known as facets) sit between the pedicle and the foramen of both sides of the vertebral body. Two facet joints are contained in both the cranial and caudal region of each vertebral body. The facet joint is an articulating, 


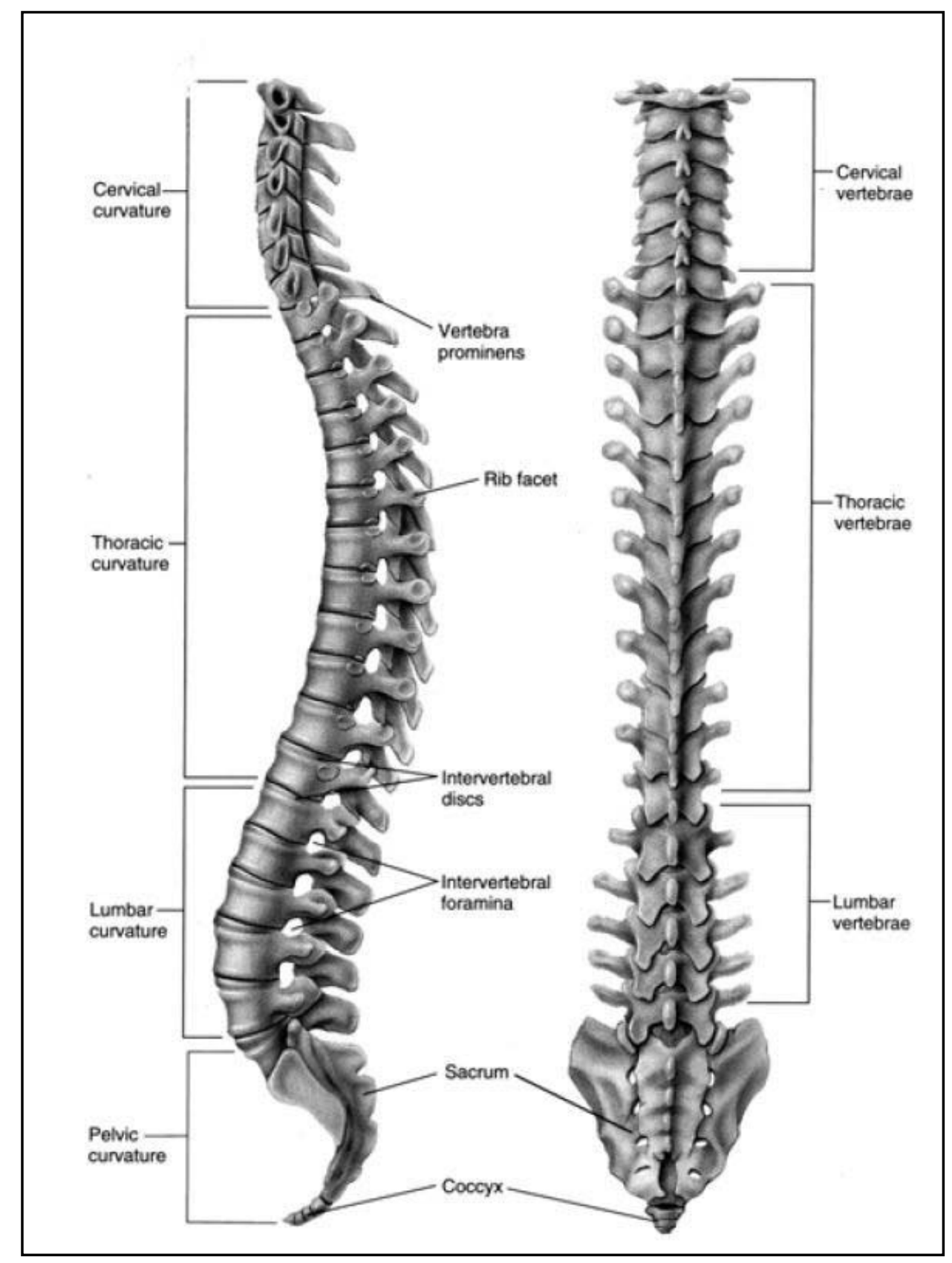

Figure 2-1 The Full Human Spinal Column. Lateral (left) and posterior (right) views. Reprinted with permission from Butler J, Lewis R, Shier D, et al. Hole's Human Anatomy \& Physiology. 9th ed. Boston: McGraw Hill, 2002. ${ }^{7}$ 


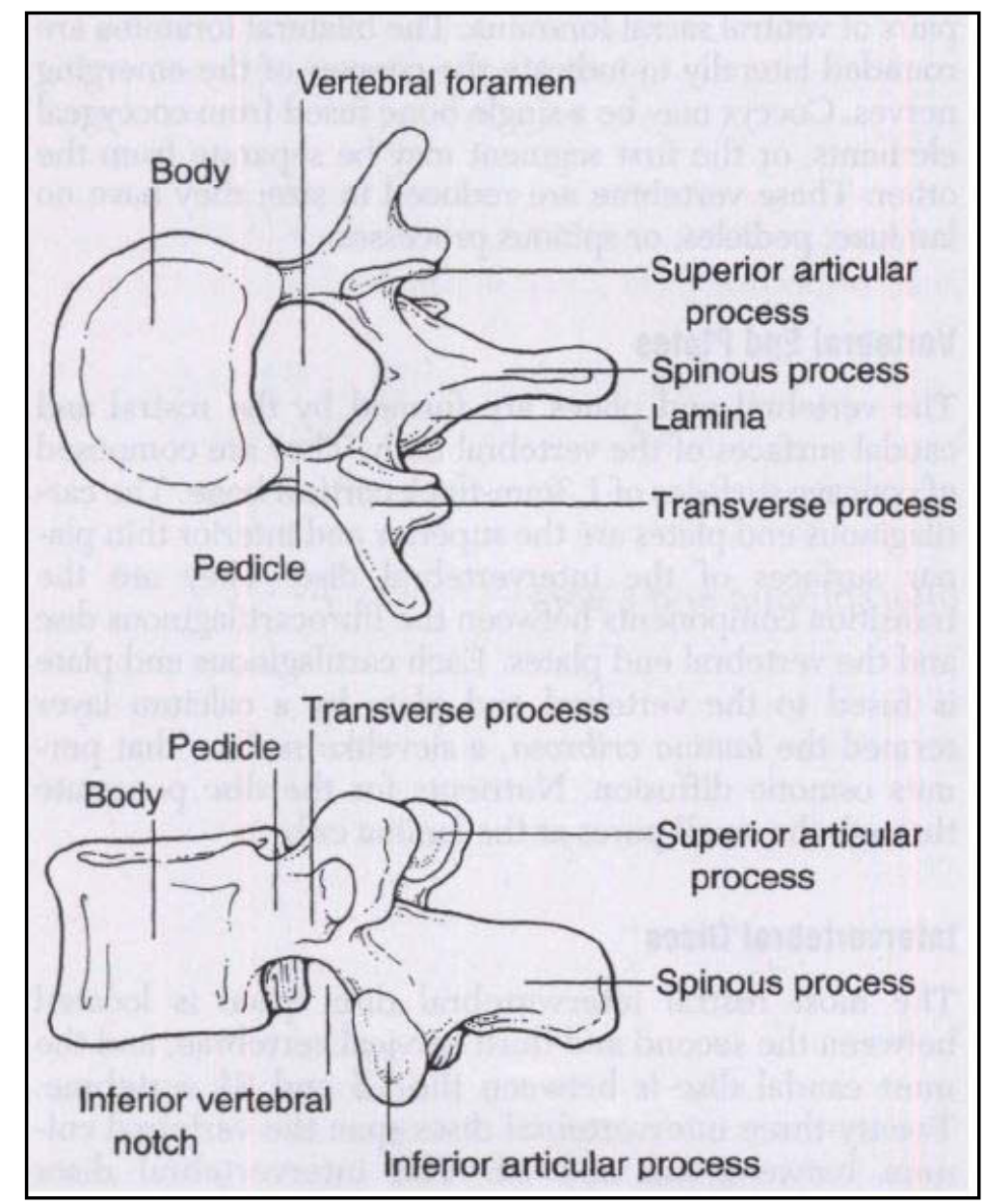

Figure 2-2 Lumbar Vertebral Body. Reprinted with permission from Benzel EC, ed. Spine Surgery: Techniques, Complications, Avoidance, and Management. 2nd ed. Philadelphia: Elsevier Churchill Livingstine, 2005. ${ }^{5}$ 
synovial joint with articular cartilage and menisci encapsulated by ligaments. ${ }^{60}$ The facet joints, which function mainly to limit motion in extension, also limit flexion and axial rotation, allow coupling between lateral bending and axial rotation, and resist shear loading. ${ }^{3}$ The facet joints are a large contributor to lower back pain due to large nerve population in the encapsulated region and high susceptibility to changes from aging and injury. ${ }^{38}$

\subsubsection{Intervertebral Disc}

As seen in Figure 2-3, the intervertebral disc is made up of three integrated regions: the inner nucleus pulposus, the outer annulus fibrosus, and the cartilaginous endplates. It is important to note that although the regions are strictly defined in the image, no such strict boundary exists anatomically. ${ }^{30}$ The cartilaginous endplates enclose the disc cranially and caudally and are considered part of the disc because the endplate and annular collagen fibers are continuous. ${ }^{30,51}$ The intervertebral discs of the spine have the same structure in different spinal regions even though the size and shape of the discs may be dissimilar. ${ }^{28}$

The nucleus pulposus is a pressurized gelatinous region made of proteoglycans (glycosaminoglycans), loose type II collagen fibrils, mineral salts, water, and cellular elements which are remains from the primitive notochord. ${ }^{39}$ The proteoglycans of the nucleus region are hydrophilic in nature, pulling water into the inner portion of the disc via osmosis. Once in the disc space, the water forms hydrogen bonds with the proteoglycans, pressurizing the region. This hydrostatic pressure (known as intradiscal pressure) allows the disc to diminish compressive loads in the spinal column by converting axial force to radial force. ${ }^{39,60}$ Water content in the nucleus can lessen depending on short-term (physical activity) or long-term (aging) factors, causing the disc space to decrease. Decreasing disc space can lead to the loss of correct joint motion and function. ${ }^{57}$

The annulus fibrosus forms the outer region of the disc and is composed of type I and type II collagen fibers. This structure allows attachment of the disc to the vertebral body and endplate, holds the nucleus material in place, and encloses the nucleus to keep it pressurized. ${ }^{60}$ As the annulus moves inward towards the nucleus pulposus, the fibers widen and the collagen content of the fibers decreases. Fibers lie in an alternating pattern, with an angle 30 or 40 degrees with respect to the horizontal. The pattern is such that adjacent fibers are nearly perpendicular to one another. This arrangement increases

the tensile strength of the annulus and prevents detachment during activity. ${ }^{51,52}$ All annulus fibers, save the outermost, attach to the cartilaginous endplates. The outermost annular layer, called Sharpey Fibers, attach directly to the vertebral body. The intervertebral disc as a whole provides resistance to axial loads and allows for and limits motion in multiple directions. ${ }^{60}$ 


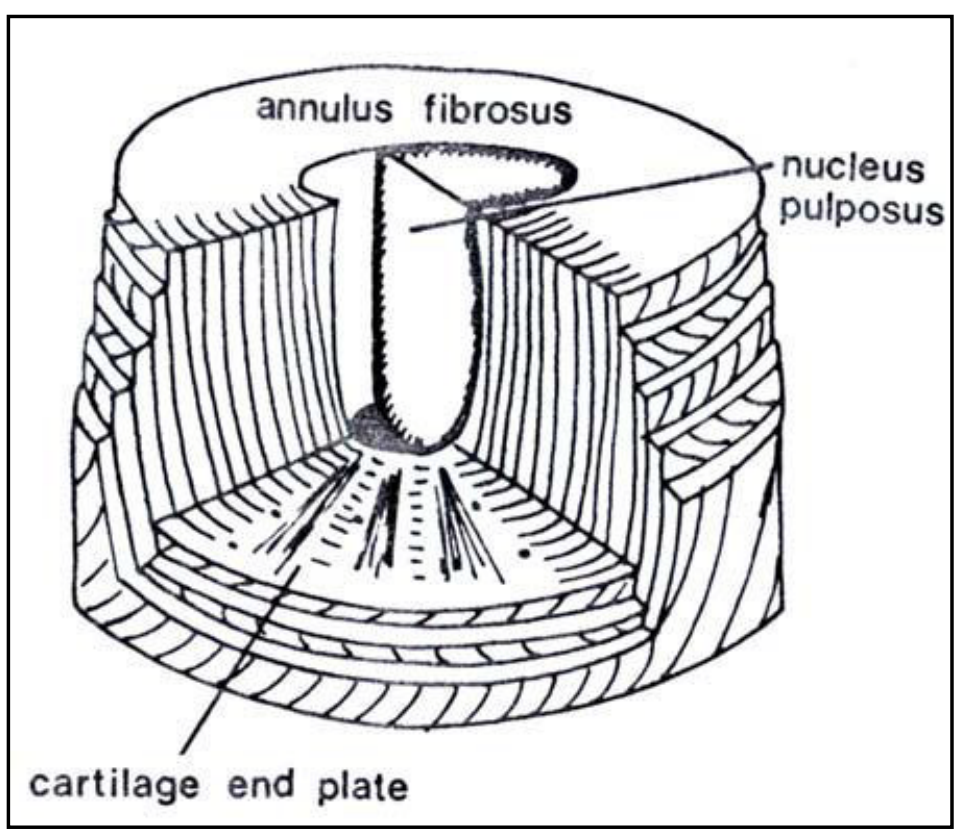

Figure 2-3 The Three Components of the Intervertebral Disc. Reprinted with permission from Humzah MD, Soames RW. Human intervertebral disc: structure and function. The Anatomical Record 1988;220:337-356. ${ }^{30}$ 


\subsubsection{Ligaments}

Ligaments are bands of fibrous connective tissue joining bones or cartilaginous structures. Ligaments provide stability to joints by facilitating or limiting motion. The structure of a ligament consists of unidirectional collagen fibers for strength and resistance and unidirectional elastin fibers for flexibility and elongation. Ligaments are only active in tensile directions, otherwise they buckle. The spinal column consists of various ligaments that provide stability by limiting motion in certain directions. The ligaments of the lumbar spine can be seen in Figure 2-4. ${ }^{60}$

While most spinal ligaments are only active across individual MSUs, there are two ligaments that span the entire spinal column: the anterior longitudinal ligament (ALL) and the posterior longitudinal ligament (PLL). The ALL is a broad ligament that spans the anterior spinal column. It attaches to the anterior vertebral bodies via a loose connection to the periosteum and to the intervertebral discs via a strong connection to the outer annular tissue. The ALL is broader while connected to the vertebral body than to the disc. The PLL runs at the posterior of the vertebral bodies and anterior of the spinal canal. It contains similar attachments to the vertebral bodies and discs but is broader at the discs than at the body. 8,60

The ligaments that attach only between individual MSUs are the ligamentum flavum, the facet capsular ligaments, the intertransverse ligaments, and the interspinous and supraspinous ligaments. The ligamentum flavum, along with the PLL, surround the spinal cord within the spinal canal. This ligament attaches to the anterior lamina of the superior vertebral body and the superior lamina of the inferior vertebral body. It is known as the yellow ligament and is considered the most elastic in the human body. This elasticity is necessary so the ligament will not pinch the spinal cord during swift flexion to extension motions. The elasticity of the ligamentum flavum is clinically significant because it allows for over-distraction during surgery without permanent damage. ${ }^{5,60}$

The facet capsular ligaments are perpendicular to the joint itself and are stiffer in the lumbar spine than in the cervical or thoracic regions. Intertranverse ligaments span between the lateral transverse processes. ${ }^{5}$ The supraspinous ligaments span between the spinous processes and range from the inferior-dorsal region of the cranial process to the superior-ventral region of the caudal process. These ligaments attach at points from the nerve root to the apex of the processes. ${ }^{2}$ Finally, the supraspinous ligaments connect the most posterior regions of the spinous processes. ${ }^{5}$

\subsection{Spine Mechanics}

\subsubsection{Intervertebral Disc Mechanics}

The hydrostatic pressure of the intervertebral disc allows the disc to convert compressive axial loads into radial loads that act on the annulus. This allows the disc to 




Figure 2-4 Ligaments of the Lumbar Spine. Reprinted with permission from Benzel EC, ed. Spine Surgery: Techniques, Complications, Avoidance, and Management. 2nd ed. Philadelphia: Elsevier Churchill Livingstine, $2005{ }^{5}$ 
act as a shock absorber and balance external forces that would otherwise create larger loads on the vertebral endplates. This effect is illustrated in Figure 2-5. The nucleus pressure balances and distributes forces throughout the spinal column and helps maintain normal join motion. ${ }^{60}$

Because the disc contains both a pressurized region (nucleus) and a tensile resistant region (annulus), it is perfectly suited to provide stability during normal sagittal and lateral bending motions. Figure 2-6 demonstrates how during flexion, the posterior region of the disc is put in tension and the anterior region is compressed. The opposite scenario holds true for extension, and lateral bending motion works in much the same way. In this way, loads are naturally balanced and the joint remains stable. ${ }^{60}$

\subsubsection{Basic Spine Mechanics}

Loads of the in-vivo lumbar spine are caused mainly by the weight of the upper torso, muscle activity, and external loads. Figure 2-7 presents a simplified picture of trunk weight acting on the lumbar spine. The variable Fbw is the body weight force that causes a moment at each spinal level. Force (in this case, body weight) multiplied by the perpendicular distance to the point of action (in this case, the center of the disc between the L5 and S1 vertebral bodies) produces the rotational moment seen in the figure. In reality, body weight acts on a line from the auricle of the ear to the femoral head. ${ }^{7}$ This line of action has been moved further posterior in Figure 2-7 for clarity in the image. Because the spine has a natural curvature as discussed in Section 2.1, the perpendicular offset distance will be different at each spinal level, causing there to be a different bending moment at each spinal level.

Fbw has an axial component that acts perpendicular to the superior endplate of the disc and a shear component that acts along the disc. These component forces will depend on the angle the disc makes with respect to the horizontal and is shown as theta $(\theta)$ in Figure 2-7. Knowing this angle, the following force components can be calculated:

$$
\begin{aligned}
& F_{A}=F_{B W} \times \cos \theta \\
& F_{S}=F_{B W} \times \sin \theta
\end{aligned}
$$

As the angle $\theta$ increases, such as during a flexion motion, more of Fbw is converted to a shear force along the disc. This is especially important at the lower lumbar levels, where initial mean disc angles are greater than at more cranial levels. While the axial component of the force is resisted by the disc, it is thought that the shear forces are resisted by the facets. ${ }^{8,60}$ Dickey and Gillespie used a porcine model to show that during extension, $72 \%$ of moment is resisted by the facets, whereas during flexion the moment resistance is only $20 \% .{ }^{19}$ During flexion, the disc and posterior ligaments resist most of the moment. Trauma, disease, surgery, and simply aging can all have a dramatic affect on the structure of the spine, leading to changes in spinal mechanics. 


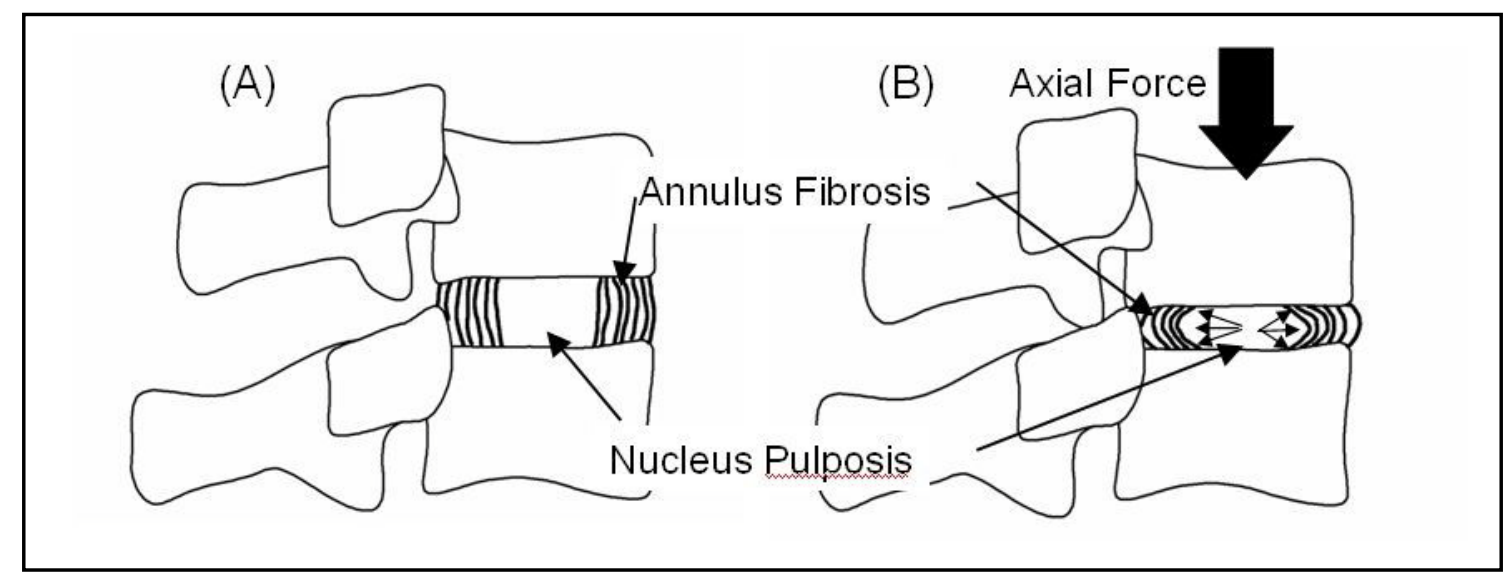

Figure 2-5 Disc Converting Axial to Radial Force. Pressure of intervertebral disc (A) under no external load and (B) when subjected to an axial force. 


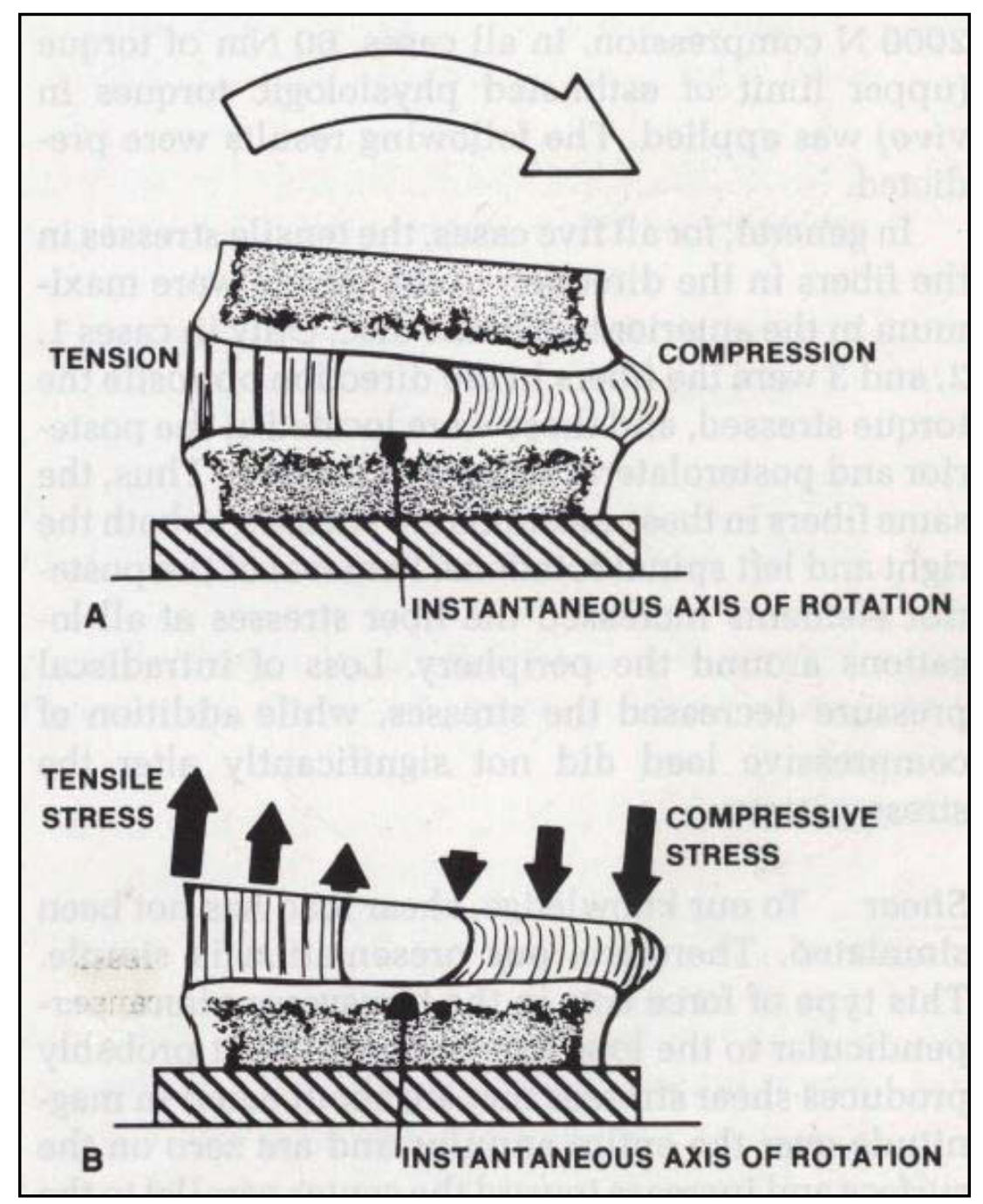

Figure 2-6 Disc Balancing Loads During Bending. The disc balances (A) loads and (B) stresses as the spine undergoes bending motions. Reprinted with permission from White AA, Panjabi MM. Clinical Biomechanics of the Spine. 2nd ed. Philadelphia: J.B. Lippincott Co., $1990 .^{60}$ 


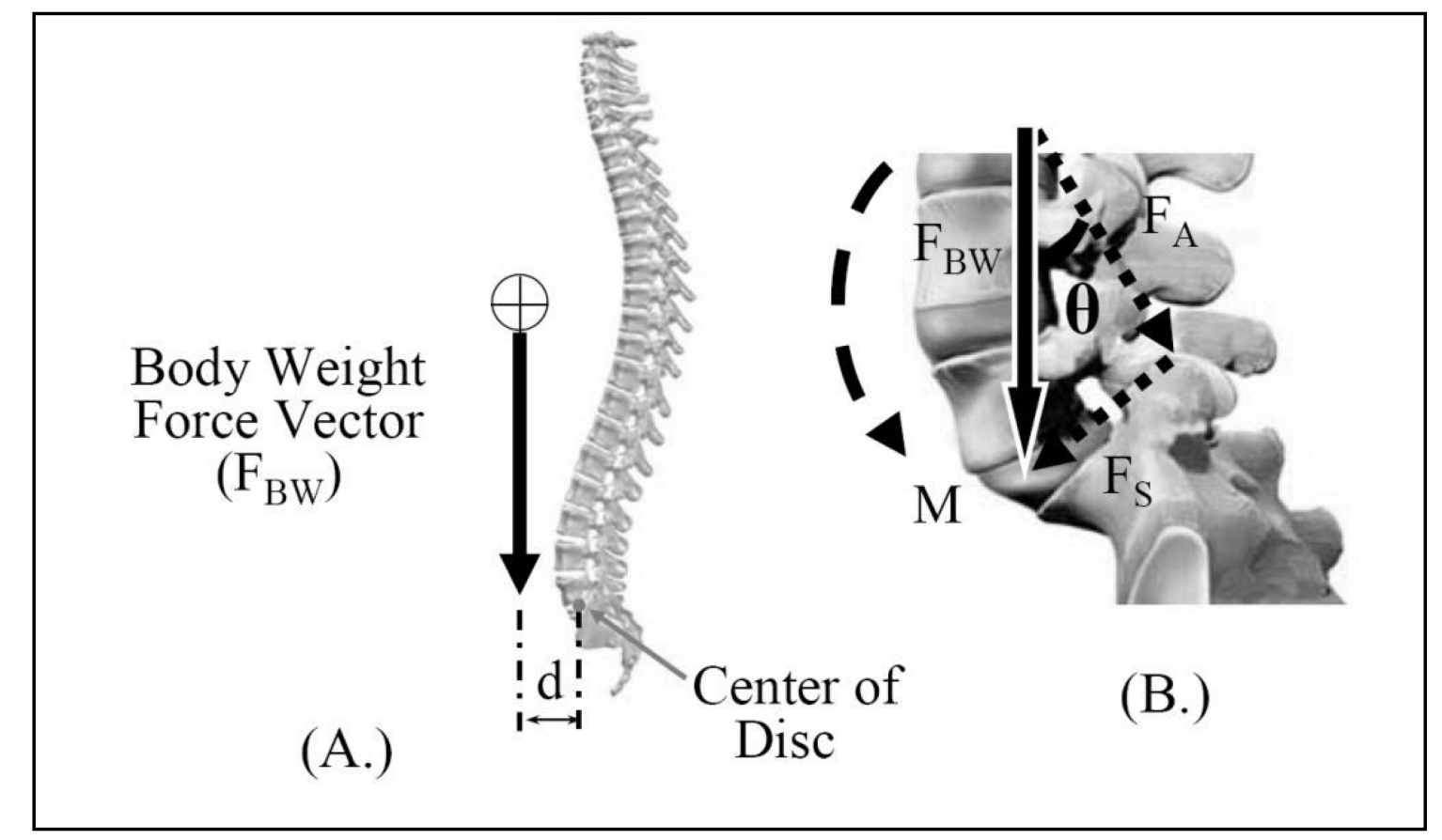

Figure 2-7 Mechanics of the Lumbar Spine. The body weight force vector (Fbw) shown in (A) induces a vertical force (Fbw) and a bending moment $(\mathrm{M}=\mathrm{Fbw} * \mathrm{~d})$ at eh L5-S1 level (B). The vertical force has two components: a shear force (Fs) which acts along the plane of the disc and an axial force $(\mathrm{Fa})$ which acts normal to the disc. As the angle of bending changes, both the force and moment values will vary. Reprinted with permission Zufelt N. A kinematics-based testing protocol to study the mechanics of the human lumbar spine. Master's Thesis. The University of Tennessee Health Science Center, 2008. ${ }^{68}$ 
Anything that limits the spine's effectiveness with regards to load sharing and stability is of chief concern clinically. ${ }^{8}$

\subsection{Spine Kinematics}

Kinematics is the study of rigid body motion. For spine biomechanics, the vertebral bodies are discussed as rigid bodies, and the kinematic characteristics of MSUs are given as the superior body with respect to the inferior body. Spinal MSUs have six degrees of freedom as shown in Figure 2-8: translation along three orthogonal axes and rotation about these axes. The neutral zone and range of motion are two parameters often discusses in regards to spinal kinematics. ${ }^{60}$

\subsubsection{Neutral Zone}

The neutral zone was originally described by Panjabi as "The region of high flexibility or laxity around the neutral position." Figure 2-9 shows the neutral zone as the area where an increase in deformation leads to only a small change in load. The neutral zone is associated with in vivo observations such as degenerative disc disease (DDD), trauma, fracture, muscle tone, and spinal fixation. ${ }^{43}$ Additionally, the neutral zone has been proposed as a better indicator of spine stability. ${ }^{45}$ However, because load ranges are so small for neutral zone measurements, this parameter does not effectively characterize the effects of devices on the spine.

\subsubsection{Range of Motion}

Range of motion (ROM) is used to diagnose pathologies in vivo, and it is the most commonly reported kinematic characteristic of in vitro testing protocols. $9,15,17,18,25,34,47,58$ $\mathrm{ROM}$ is either reported as the rotation of the entire spinal section (cervical or lumbar), or the relative rotation of one vertebral body with respect to another (MSU rotation). It is common to report ROM data for harvested, non-altered specimen to be compared to ROM data for the same specimen instrumented or altered in some way. ${ }^{17,21}$ Clinically, lower lumbar levels are of more interest due to their higher motions and therefore higher rates of disease. ${ }^{60}$ In Figure 2-9, the ROM is the total of the neutral and elastic zones.

\subsection{Spine Biomechanical Testing}

Methods of biomechanically testing the spine grew out of modifications to biomechanical tests of synovial joints. In 1988, Panjabi et al categorized spinal biomechanical testing into two groups: flexibility protocols (load-controlled) or stiffness protocols (displacement controlled). For a flexibility protocol, some load (linear, rotational, or a combination) is applied to a specimen and the resulting displacement 


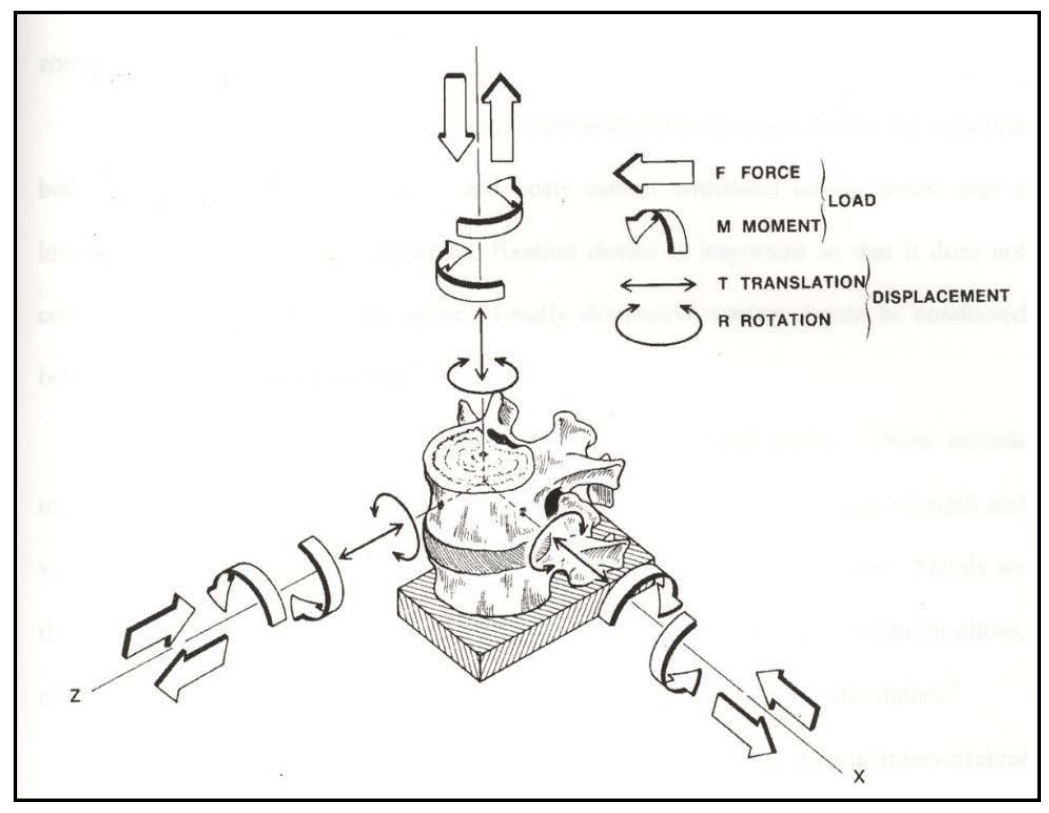

Figure 2-8 Six Degrees of Freedom in Spinal MSU. Translation along and rotation about the three principal orthogonal axes fully describe the kinematic range of the MSU. Reprinted with permission from White AA, Panjabi MM. Clinical Biomechanics of the Spine. 2nd ed. Philadelphia: J.B. Lippincott Co., $1990{ }^{60}$

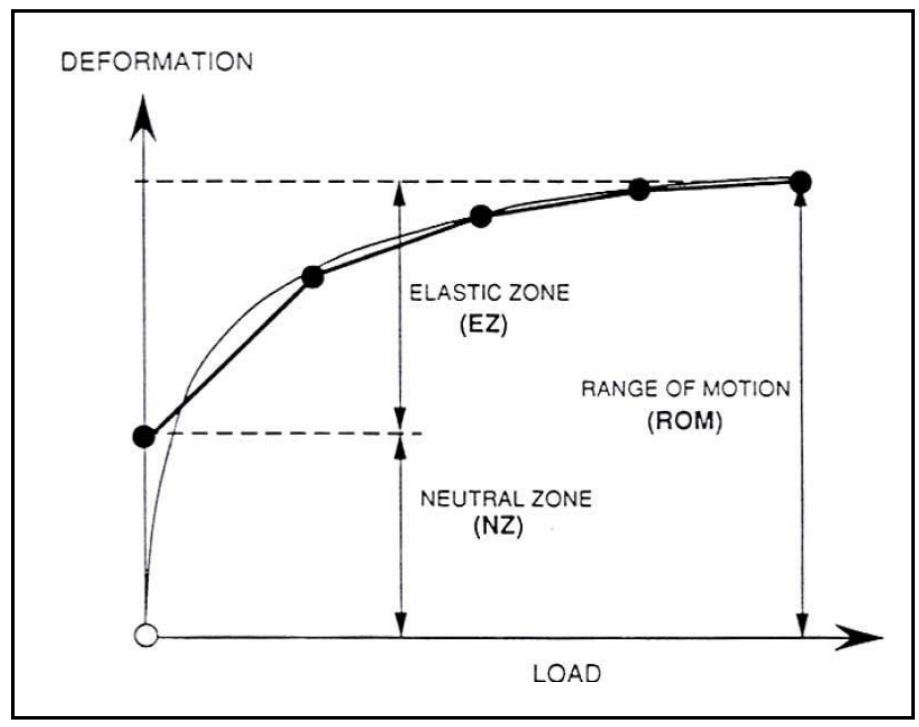

Figure 2-9 Neutral and Elastic Zones of Spinal Motion. Flexibility plot (Deformation vs. Load) illustrating the neutral and elastic zones of motion. Reprinted with permission from Panjabi MM. The stabilizing system of the spine. Part II: Neutral Zone and Instability Hypothesis. Journal of Spinal Disorders 1992;5:390-396. ${ }^{43}$ 
(translation, rotation, or a combination) is measured. Stiffness protocols work by applying a displacement to a specimen and measuring the resulting load. ${ }^{42}$

Which test method is proper is a state of contention amongst spine biomechanics researchers. Supporters of the flexibility protocol point out that load-control, specifically pure moment testing methods, offer the greatest control over the complex variables in spine testing. Because pure moments induce a similar loading profile at every spine level, testing this way allows comparison between surgical techniques and instrumentation cited previously in the literature and allows comparison between single and multi-level studies. Proponents of flexibility testing believe the uniformity between tests will allow newer instruments and implants to be directly compared to those already approved by the FDA and other world organizations. Only after such tests have been conducted would stiffness protocols be used to further study devices. Supporters of the stiffness protocol main concern with the flexibility protocol is that it does not replicate invivo conditions and therefore does not induce a response that is valid clinically. They argue that the use of displacement control allows for better replication of in-vivo conditions, which will allow for a better understanding of clinical responses to surgical techniques and devices. ${ }^{27}$ In the next two sections, both protocols will be further discussed.

\subsubsection{Flexibility Protocol (Load-Control)}

The flexibility protocol commonly involves the application of a pure rotational input to a free and unsupported end of a spinal segment. A typical setup can be seen in Figure 2-10. The superior end of the spinal segment is attached to a pulley. A cable system twists the specimen while simultaneously minimizing any axial or shear loading experienced by the segment. ${ }^{47}$ Other systems include dead weights suspended on rails attached to the superior spinal segment ${ }^{26}$ and a system of pneumatic actuators and gliding rails. $^{42}$

A typical flexibility study involves the comparison between harvested (intact) and treated cadaveric specimen in one or more of the three spinal degrees of rotation (flexion/extension, lateral bending, and axial rotation). ${ }^{45,48,59}$ Typically, studies involving rotation around more than one principal axis involve removing the specimen from the test frame between individual tests. Recently, however, multi-axis testing machines have been developed allowing pure moment testing around multiple axes without specimen removal. Such testing platforms generally only have the capacity to drive one axis at a time, making them unable to induce combined loading scenarios that are often seen invivo. $^{13,47,61}$

With the influx of newer non-fusion devices to the market, many attempts to modify the traditional flexibility protocols have been made. ${ }^{13,32,36}$ One widely-known modification to the protocol is Panjabi"s "hybrid protocol" used to study adjacent level effects (ALE). ALE is defined by Panjabi as the "long-term accelerated degeneration at adjacent-levels due to spinal fusion surgery." The same effects should also be studied in 


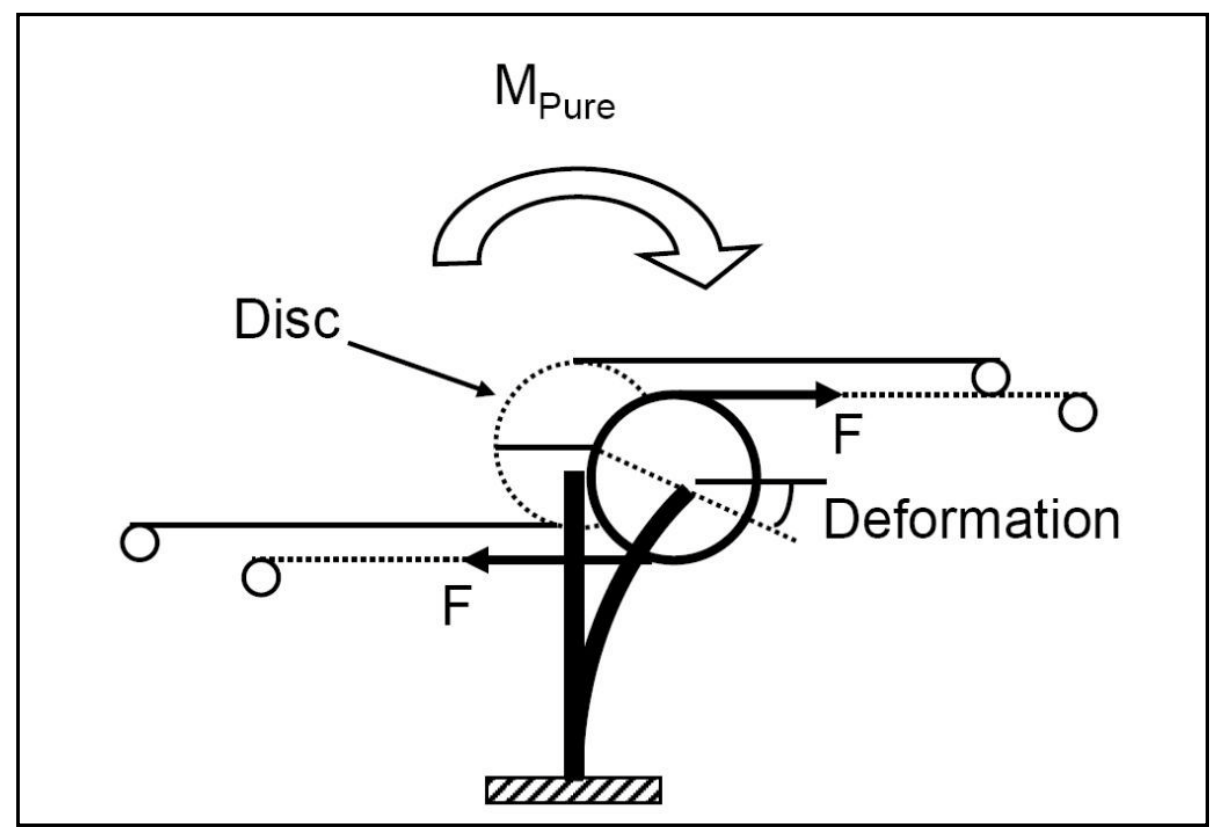

Figure 2-10 Illustration of Common Pure Moment Setup. In this setup, the pure moment is induced using parallel pulleys attached to the pictured disc. Adapted with permission from Panjabi MM. Hybrid multidirectional test method to evaluate spinal adjacent-level effects. Clinical Biomechanics 2007;22:257-265. ${ }^{46}$ 
non-fusion instrumentation. ${ }^{46}$ Although widely used, hybrid protocol is not without detractors. The main argument against the protocol is the underlying assumption that a patient will move their spine with the same ROM after a fusion or total disc arthroplasty (TDA) surgery. Many question this assumption, and there is clinical evidence to suggest that patients' ROM decreases post-surgery. This is the main contributor to some researchers' belief that the hybrid protocol does not produce clinically relevant data and is a source of controversy in the biomechanics community. ${ }^{11}$

Another popular modification to the flexibility protocol is the use of a follower load. ${ }^{24,44,55}$ A concern among researchers is that a follower load might artificially stabilize the spine and not show an appropriate response from a spinal segment to a particular technique or device. ${ }^{16,48}$ The two main benefits to flexibility testing are the relative ease of using the protocol and the ability to make standardized comparisons to previous studies. One major limitation is that because physiological spine loading cannot be measured non-invasively, assumptions must be made about load limits during testing. Another limitation is the fact that during multi-body testing, the same amount of moment is applied to each level, a scenario that is not physiological, as discussed in Section 2.2.2. Finally, because the use of pure moments minimizes shear load, their use makes it impossible to study how different devices affect shear loading at the operative and adjacent levels. ${ }^{27,42}$

\subsubsection{Stiffness Protocol (Displacement-Control)}

The stiffness protocol refers to a testing method where a specimen is displaced (translation, rotation, or a combination) and resulting load values are measured. Stiffness protocols have been used to study both fusion and non-fusion devices. ${ }^{17,31,33}$ Theoretically, the stiffness protocol should allow researchers to exactly mimic in-vivo behavior. Since in-vivo MSU displacements can be measured non-invasively, such displacements could be directly integrated into in-vitro models. Stiffness protocols can often be run by commercial test frames with a single degree of freedom. One such similar implementation of the protocol is outlined below.

DiAngelo et al developed a stiffness protocol using a horizontally mounted lever arm acted on by a vertical actuator, an illustration of which can be seen in Figure 2-11. In this setup, the horizontal lever arm is attached to the top pot of the specimen by a constrained fixture and to the vertical actuator by a roller bearing. The slider bearing allows the compressive force from the vertical actuator to act normal to the specimen's top pot, and bending is produced by a combination of the compressive force and an induced bending moment. Using this method, a physiologic bending response is induced within the spine. ${ }^{17}$

A second example of the stiffness protocol is the application of a constrained rotation input to a specimen outlined by Panjabi. ${ }^{46}$ An investigator using such a protocol would need to carefully consider the placement of the center of rotation, as outlined by Bonin $^{6}$ and Zufelt. ${ }^{68}$ 


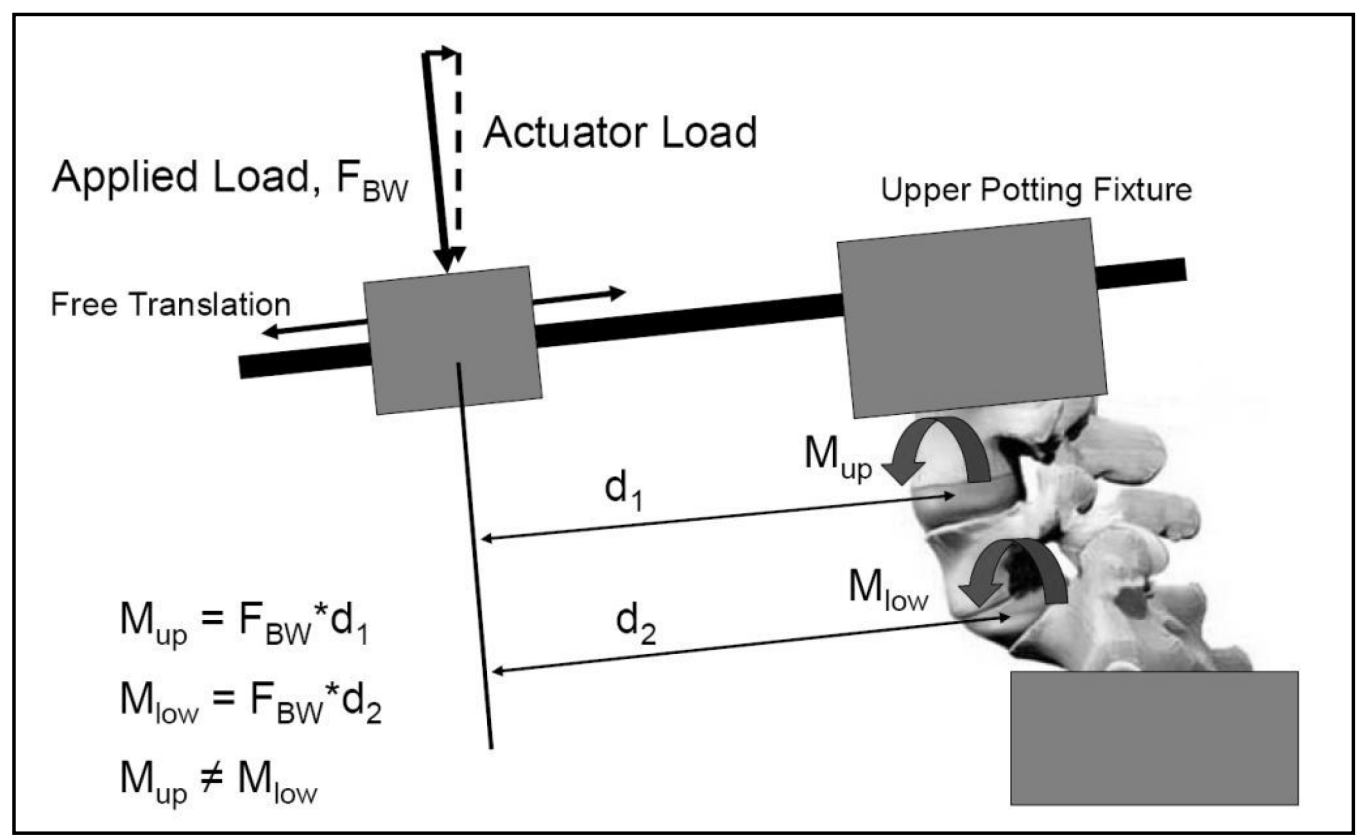

Figure 2-11 Illustration of Eccentric Load Protocol Setup. A horizontal moment arm attaches the specimen's top pot to a vertical actuator via a roller bearing. The roller bearing is unconstrained along the moment arm and allows the compressive force to act normal to the top pot of the specimen. No shear force should be applied. Reprinted with permission from Zufelt N. A kinematics-based testing protocol to study the mechanics of the human lumbar spine. Master's Thesis. The University of Tennessee Health Science Center, 2008. ${ }^{68}$ 
Use of the stiffness protocol is limited due to the difficulty in obtaining load data at individual segments during multi-body testing. Because the loading of the specimen is not evenly distributed, more than a single load cell at either end of the specimen is needed to get accurate load data during such testing. Also, the testing of very stiff specimen is difficult with stiffness protocols because small changes in displacement lead to large changes in load magnitudes. ${ }^{27}$

\subsection{Clinical Relevance of IAR Location}

The COR for a motion segment is the point about which the cranial vertebral body rotates about relative to the caudal body. For small rotations, all physiological points maintain a constant distance from the COR throughout rotation. This parameter is often referred to as the IAR, which is the axis that passes through the COR perpendicular to the plane of motion. 6,68

For in-vivo testing, bony landmarks on radiographs are used to calculate the IAR for an individual patient. Patient's need to be subjected to minimal radiation, so generally only three radiographs are used: neutral position, extreme flexion, and extreme extension. ${ }^{50,49,53,66}$ Although some researchers argue this is enough data for an accurate calculation, errors associated with choosing bony landmarks in such a small number of radiographs of varying quality has lead to skepticism with the technique. ${ }^{41,56}$ In-vitro calculation of the IAR involves measurements with specimen mounted optical diodes connected to a camera tracking system. The inherent error associated with camera tracking systems makes this a less than ideal method. ${ }^{12,14}$

Many researchers associate IAR positions with indications for diseases and trauma related to lower lumbar segments. Unfortunately, with the disagreements regarding both the IAR locations and how to calculate them, doctors are hesitant to make IAR values a part of the diagnosis process. As better, more accurate data become available, clinicians will be able to use these characteristics of the spine in patient care. $^{53,54}$ 


\section{Chapter 3 - Creation of a Pure Moment Protocol for Studying Instantaneous Axis of Rotation Using a Programmable Spine Robot}

\subsection{Introduction}

The development of cadaveric biomechanical testing has led to greater understanding of human spine function. These tests protocols have also allowed orthopedic companies to test techniques and instruments for desired anatomical responses and restorative value. Testing of a harvested condition along with an instrumented condition under the same parameters yields data that identify how clinical techniques and instruments influence spinal motion and load. Biomechanical testing methodologies generally fall under two categories: (1) Displacement Control - testing where spine motion is controlled for harvested and instrumented conditions and load values are analyzed; (2) Load Control - testing where loads (forces or moments) are controlled for harvested and instrumented conditions and motion is analyzed. Both methodologies are commonly used in the biomechanical testing community and each has strengths and weaknesses. ${ }^{17,27,42}$

A common method of load-controlled mechanical testing of human cadaveric spines is the pure moment protocol. The pure moment testing method involves the application of a pure rotational load to a free and unsupported end of spinal segment. The opposite end of the spinal segment is held stationary via fixation to the base of the testing platform. If the top of the spinal segment is supported by the testing apparatus, then a pure moment can be applied to the spine by minimizing the axial and shear forces that the segment experiences. ${ }^{27,42}$ The pure moment response is generally induced using one of several techniques: deadweights on rails attached to the superior spine end ${ }^{26}$, pneumatic actuators with sliding rails ${ }^{1,42,46}$, or a uniaxial hydraulic test frame attached to a mobile cable and pulley system. ${ }^{9,10}$ Common testing platforms for pure moment protocols include equipment that is suboptimal for reducing off-axis loading. Off-axis loads contribute to the moment value of a given test, leading to testing errors. Our initial motivation for this study was to use the programmable Spine Robot at the University of Tennessee Health Science Center to create a pure moment testing protocol that significantly reduced off-axis loads and moment errors relative to other common test platforms.

Specimens are usually tested in one or more of sagittal plane bending (flexion/extension), coronal plane bending (lateral bending), and transverse plane rotation (axial rotation) modes. Pure moment methods should ideally induce the same load conditions for every test, making testing of new instruments and techniques easily comparable to older studies. The pure moment protocol is often used to compare spinal fusion instrumentation, and has also been used to evaluate non-fusion instrumentation (disc arthroplasty devices). ${ }^{62,63}$

While many of the studies dealing with load-control protocols report range of motion and flexibility data, few report data on COR values. The COR for a motion 
segment is the point about which the cranial vertebral body rotates about relative to the caudal body. For small rotations, all physiological points maintain a constant distance from the COR throughout rotation. This parameter is often referred to as the IAR, which is the axis that passes through the COR perpendicular to the plane of motion. ${ }^{6,68}$ For many in-vivo studies, radiographic measurements are taken at neutral position, extreme flexion and extreme extension. ${ }^{50,49,53,66}$ The rationale for such little measurement is to limit patient exposure to potentially harmful radiation. Studies of in-vivo and in-vitro motion have shown shifts in the IAR location throughout testing, calling into question the previously mentioned in-vivo techniques. ${ }^{41,56}$ In-vitro cervical testing has shown a moving IAR in the cervical spine in a previous study conducted in the University of Tennessee Health Science Center Joint-Implant Biomechanics Laboratory. ${ }^{6}$

There are many challenges associated with the measurement of the IAR of spinal segments with current test platforms. The limits of patient exposure to radiation and difficulty in choosing bony landmarks on varying quality radiographs create errors in invivo studies. In-vitro studies are limited by the error associated with common camera tracking systems. ${ }^{12,14}$ The IAR location in the lumbar spine has been correlated with degenerative disc disease, spondylolisthesis, and trauma, indicating that it is an important clinical measurement. ${ }^{53,54}$ The high degree of motion control and measurement accuracy associated with the Spine Robot makes it an ideal testing platform for calculating the IAR for spinal segments.

The programmable Spine Robot at the University of Tennessee Health Science Center has the capacity to evaluate different testing methodologies and allows the development of more advanced testing protocols in spine biomechanics. The goal of this study was to use the Spine Robot to simulate a pure moment protocol for flexion/extension and left/right lateral bending testing on a single-level lumbar MSU. Developing a pure moment protocol gives the lab the ability to compare data with those reported in the literature and is a starting point for more sophisticated testing. Additionally, motion data during pure moment testing was used to calculate the IAR of the specimens.

\subsection{Materials and Methods}

Eight fresh human cadaveric lumbar spines were procured. All specimens were screened with anterior/posterior and lateral radiographs to exclude those with marked degenerative changes or significant osteoporosis. Bone density measurements were not done. The average age of the specimens was $59.7 \pm 11.7$ years. Specimen were dissected into L4-L5 single-level MSUs and potted in a bismuth alloy to allow proper interface with the Spine Robot. Specimens were potted with the top and bottom bismuth plates parallel to allow for a repeatable mounting procedure as shown in Figure 3-1. The specimens were mounted and tested in the programmable Spine Robot.

The Spine Robot has vertical and horizontal linear actuators and two rotational motors giving it four degrees of freedom, as illustrated in Figure 3-2. All four degrees of 


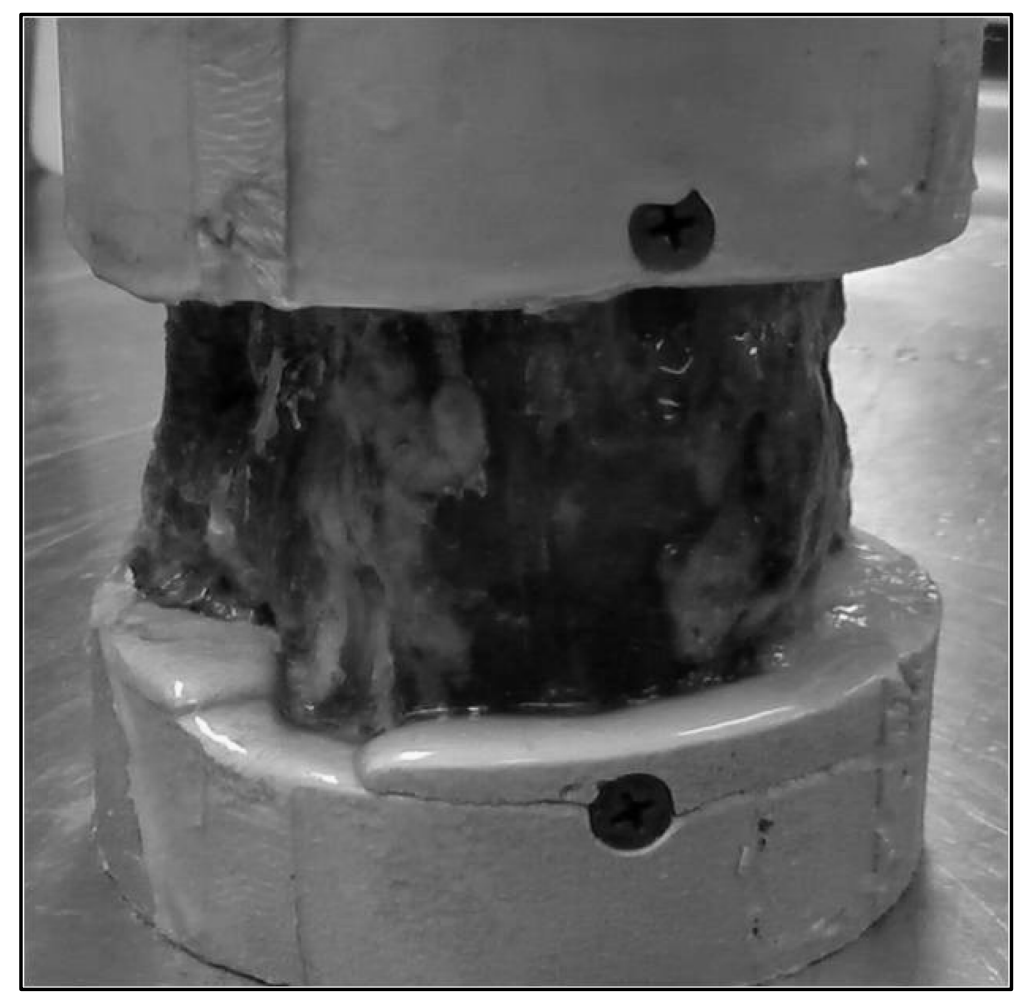

Figure 3-1 L4-L5 MSU Potted in Bismuth Alloy. The top and bottom plates are kept parallel to ensure repeatable mounting of the specimen. 


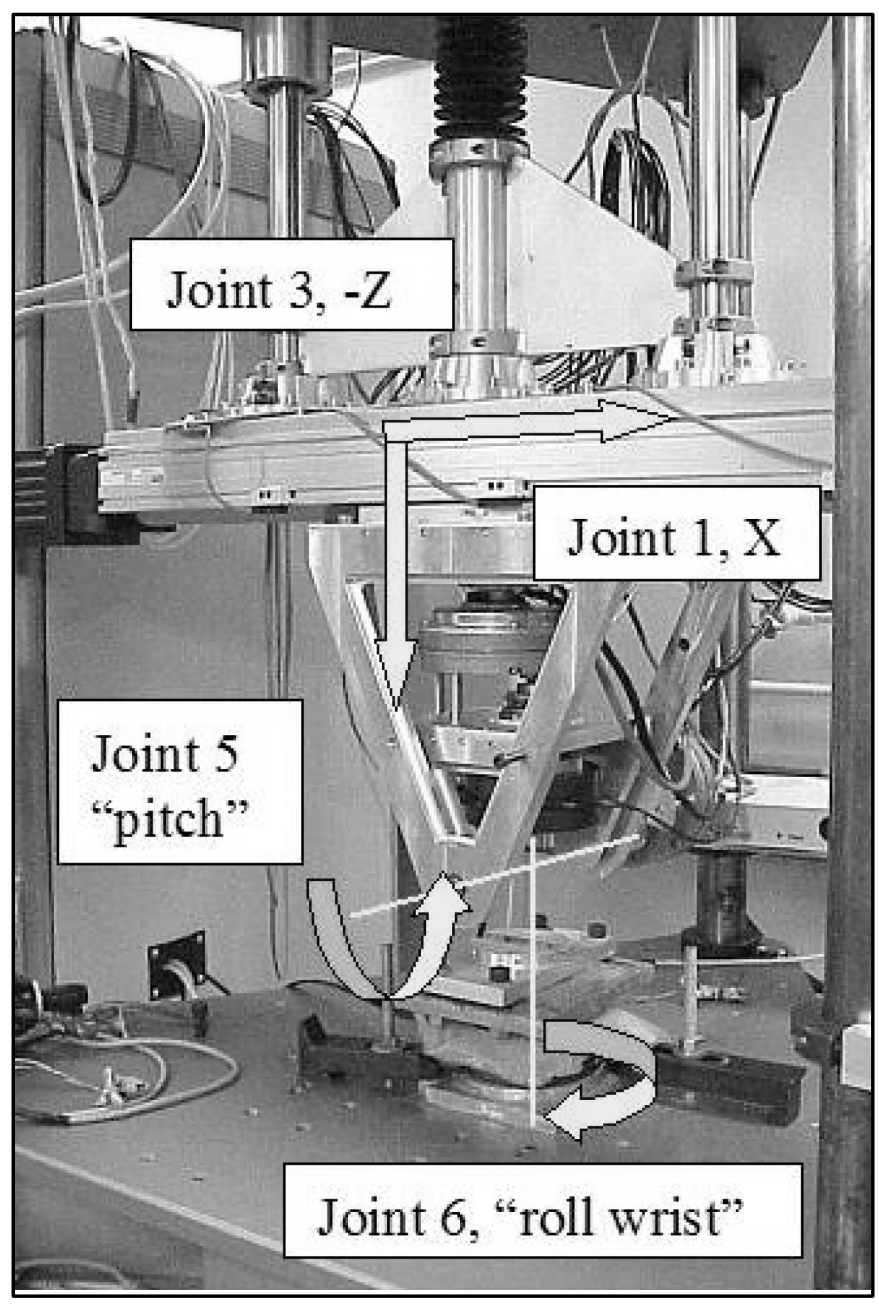

Figure 3-2 Spine Robot with Gimbal Attachment. Degrees of freedom are illustrated with arrows. A six axis load cell, located in the gimbal assembly, connects to the top mounting pot of the spinal segment. Reprinted with permission from Kelly BP. A multiaxis programmable spine robot for the study of multibody spinal biomechanics using real-time hybrid force and displacement control strategies. $\mathrm{PhD}$ Dissertation. The University of Tennessee Health Science Center, 2005. ${ }^{35}$ 
freedom are independently programmable and can be operated under load control, displacement control, or a combination of both. The Spine Robot has the capacity to create coupled loading conditions, complex MSU mechanics (such as the introduction of shear forces), and fixed-path and other kinematic conditions. Relevant statistics of the various actuators of the Spine Robot are listed in Table 3-1.

A custom software control program was written such that once a specimen was mounted into the Spine Robot, the user specified the following input parameters: rotational increment, final moment, and load cell force tolerance(s). A rotational increment of 0.25 degrees was used for all testing described here.

After user input, an automated process began where the specimen rotated sagittally (for flexion/extension) or laterally (for lateral bending) the amount of the rotation increment. The first incremental rotation was about a central point along an axis midline to the intervertebral disc near the subjacent endplate.

The six degree of freedom load cell in the robot reported all net forces and moments experienced by the segment after each rotational input. Load cell values were transformed to the approximated center of rotation for the segment found by radiographic analysis of each specimen prior to testing. From these values, the Spine Robot determined the amounts of shear and axial force on the segment and offset this force using the horizontal and vertical actuator motion. Once the shear and axial forces were within the load cell tolerance range inputted by the user ( $\pm 2 \mathrm{~N}$ for all testing) the Spine Robot saved the position and forces for that rotational increment and determined if the targeted maximum moment value had been reached. If the moment was below the maximum moment value, the process repeated with another incremental rotation. As the specimen was translated in order to reduce the axial and shear forces, the center of rotation point for the following incremental rotation was also translated. The testing center of rotation shifted as the test progressed and all axial and shear values were reported relative to the axis of the disc. A flowchart of the pure moment test process can be found in Figure 3-3.

Through this automated process, a quasistatic path of minimal shear and axial loading was found for each specimen. For flexion/extension tests, specimens were rotated to an $8 \mathrm{Nm}$ limit. For lateral bending tests, the specimens were rotated to $6 \mathrm{Nm}$ and were unconstrained in axial rotation. Path finding took approximately 4-5 minutes per mode of bending test. Throughout all testing, force and moment values that were not being investigated were monitored and recorded and were not significant.

In order to calculate the IAR for each specimen, equations derived by Crisco et $\mathrm{al}^{12}$ were used. These equations use perpendicular bisectors of two vertebral points over time. Figure 3-4 shows the motion of the two points and relative position vectors. If the two points over time are labeled as A1(x1,y1), A2(x2,y2), B1(x3,y3), and B2(x4,y4), then the position vectors $\mathbf{u} \mathbf{1}$ and $\mathbf{u} \mathbf{2}$ equal:

$$
\mathbf{u 1}=(\mathrm{A} 1 \mathrm{x}-\mathrm{B} 1 \mathrm{x}) \mathrm{i}+(\mathrm{A} 1 \mathrm{y}-\mathrm{B} 1 \mathrm{y}) \mathbf{j}
$$


Table 3-1 Spine Robot Specifications.

\begin{tabular}{|c|c|c|c|c|c|}
\hline $\begin{array}{l}\text { Degree of } \\
\text { Freedom } \\
\end{array}$ & Mechanism Type & Device/Manufacturer & Description & $\begin{array}{c}\text { Range of } \\
\text { Motion }\end{array}$ & Resolution \\
\hline 1 & $\begin{array}{c}\text { Linear Ball Screw } \\
\text { Actuator }\end{array}$ & 406XR / Parker Automation & $\begin{array}{l}\text { Provides horizontal } \\
\text { translation (x-axis) }\end{array}$ & $600 \mathrm{~mm}$ & $2 \mu \mathrm{m}$ \\
\hline 2 & $\begin{array}{l}\text { Linear Roller } \\
\text { Screw Actuator }\end{array}$ & GSX-30 / Exlar Corporation & $\begin{array}{l}\text { Provides vertical } \\
\text { translation (y-axis) }\end{array}$ & $300 \mathrm{~mm}$ & $0.31 \mu \mathrm{m}$ \\
\hline 3 & $\begin{array}{l}\text { Rotary Geared } \\
\text { Servomotor }\end{array}$ & $\begin{array}{c}\text { 9FG / Kollmorgen PMI } \\
\text { Division }\end{array}$ & $\begin{array}{l}\text { Provides rotation } \\
\text { about the z-axis in the } \\
x-y \text { plane }\end{array}$ & $360^{\circ}$ & $.0045^{\circ}$ \\
\hline 4 & $\begin{array}{l}\text { Rotary Geared } \\
\text { Servomotor }\end{array}$ & $\begin{array}{l}\text { 9FG / Kollmorgen PMI } \\
\text { Division }\end{array}$ & $\begin{array}{l}\text { Provides rotation } \\
\text { about the } y \text {-axis in the } \\
\mathrm{x}-\mathrm{z} \text { plane }\end{array}$ & $360^{\circ}$ & $.0045^{\circ}$ \\
\hline
\end{tabular}

Specifications of the actuators and servomotors of the UTHSC programmable Spine Robot. ${ }^{6}$ 


\section{Control Diagram}

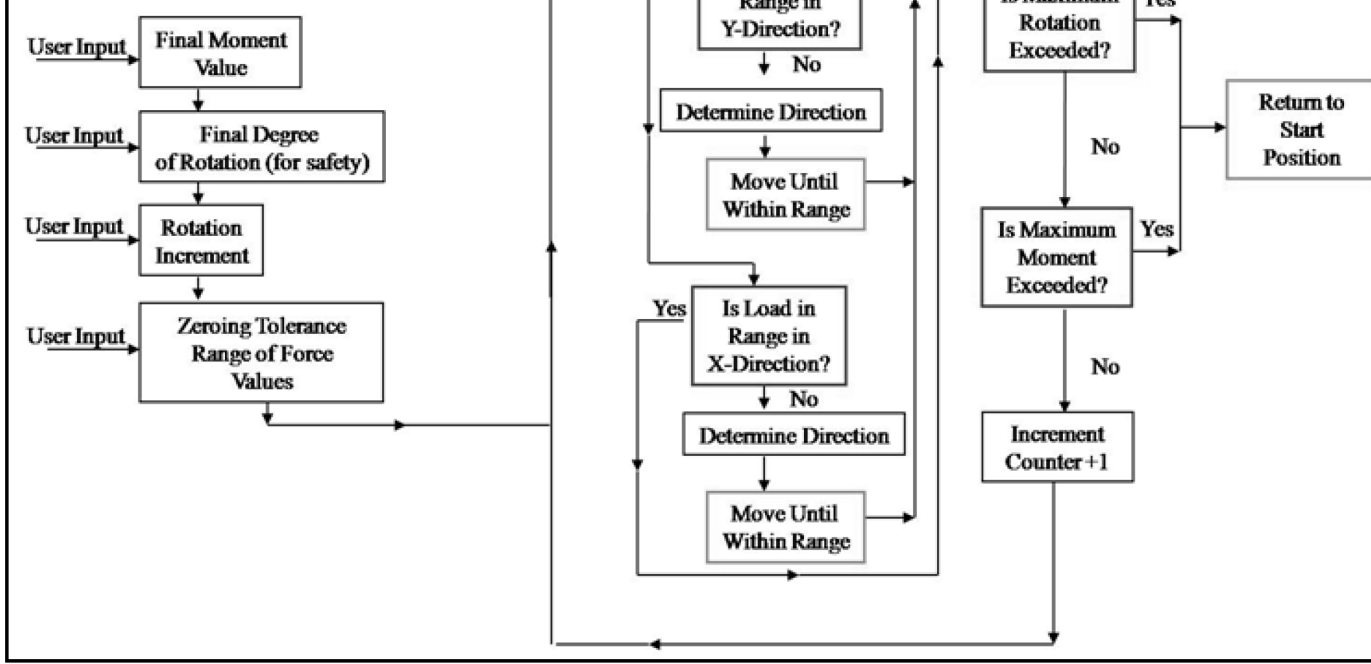

Figure 3-3 Flowchart Outlining Pure Moment Testing Protocol. 


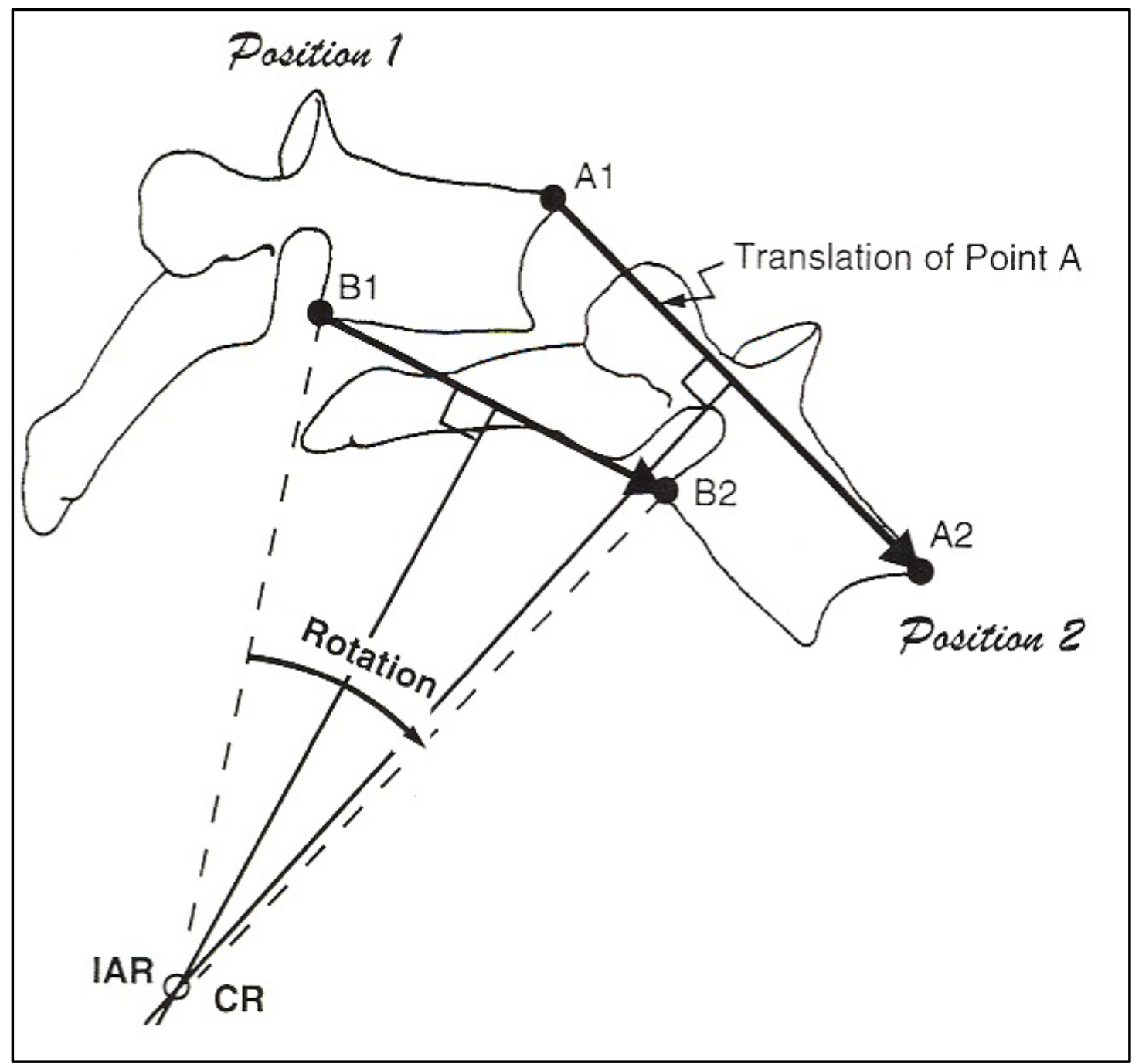

Figure 3-4 IAR Calculation. IAR is found using perpendicular bisectors of common vertebral body points between rotation intervals. Reprinted with permission from Clark CR, ed. The Cervical Spine. 3rd ed. Philadelphia: Lippincott-Raven Pub., $1998 .^{8}$ 


$$
\mathbf{u} 2=(\mathrm{A} 2 \mathrm{x}-\mathrm{B} 2 \mathrm{x}) \mathrm{i}+(\mathrm{A} 2 \mathrm{y}-\mathrm{B} 2 \mathrm{y}) \mathrm{j}
$$

and the cosine of the angle between the position vectors is:

$$
\cos \phi=\frac{u 1 \cdot u 2}{|u 1||u 2|}
$$

For this research Phi $(\Phi)$ corresponds to the pure rotation angle the cranial vertebral body of each MSU between each incremental rotation along the quasistatic path. Because the Spine robot records the exact angle and position data of the cranial MSU, the IAR of each specimen can be calculated using the following equations:

$$
\begin{aligned}
& \mathrm{X}_{\mathrm{IAR}}=\frac{1}{2} \times(\mathrm{x} 1+\mathrm{x} 2)+\frac{(\mathrm{y} 1-\mathrm{y} 2) \times \sin \phi}{2 \times(1-\cos \phi)} \\
& \mathrm{Y}_{\mathrm{IAR}}=\frac{1}{2} \times(\mathrm{y} 1+\mathrm{y} 2)+\frac{(\mathrm{x} 1-\mathrm{x} 2) \times \sin \phi}{2 \times(1-\cos \phi)}
\end{aligned}
$$

Once the IAR values were calculated, the values were compared between modes of testing (flexion/extension) with a one-way ANOVA with a Dunn's pair-wise multiple comparison test $(\mathrm{p}<0.05)$.

\subsection{Results}

In order to ensure repeatability between tests, one control specimen was tested using the flexion pure moment protocol eight times by four consecutive tests on two consecutive days. As seen in Figure 3-5, the data derived from the tests showed a high degree of repeatability. The figure shows the loading portion of flexion tests only. This control specimen was not used in any other tests and is not a part of any subsequent data. Results for specimen rotation in the four modes of bending can be seen in Table 3-2.

Flexibility curves were generated for each specimen in each bending mode. These curves can be seen in Figure 3-6. The stiffness curves exhibited a pattern that is common for viscoelastic tissue testing.

The average shear and axial forces found during specimen testing can be found in Table 3-3. A negative shear force represents an anterior shear for flexion/extension testing and a shear in the direction of rotation for lateral bending tests. A negative axial force represents a compressive force, while a positive axial force represents a tensile force.

After each incremental rotation the spine robot saved data for shear and axial forces experienced by the spine. Figure 3-7 illustrates the shear forces throughout rotation for the four modes of bending. Figure 3-8 illustrates the axial forces throughout 


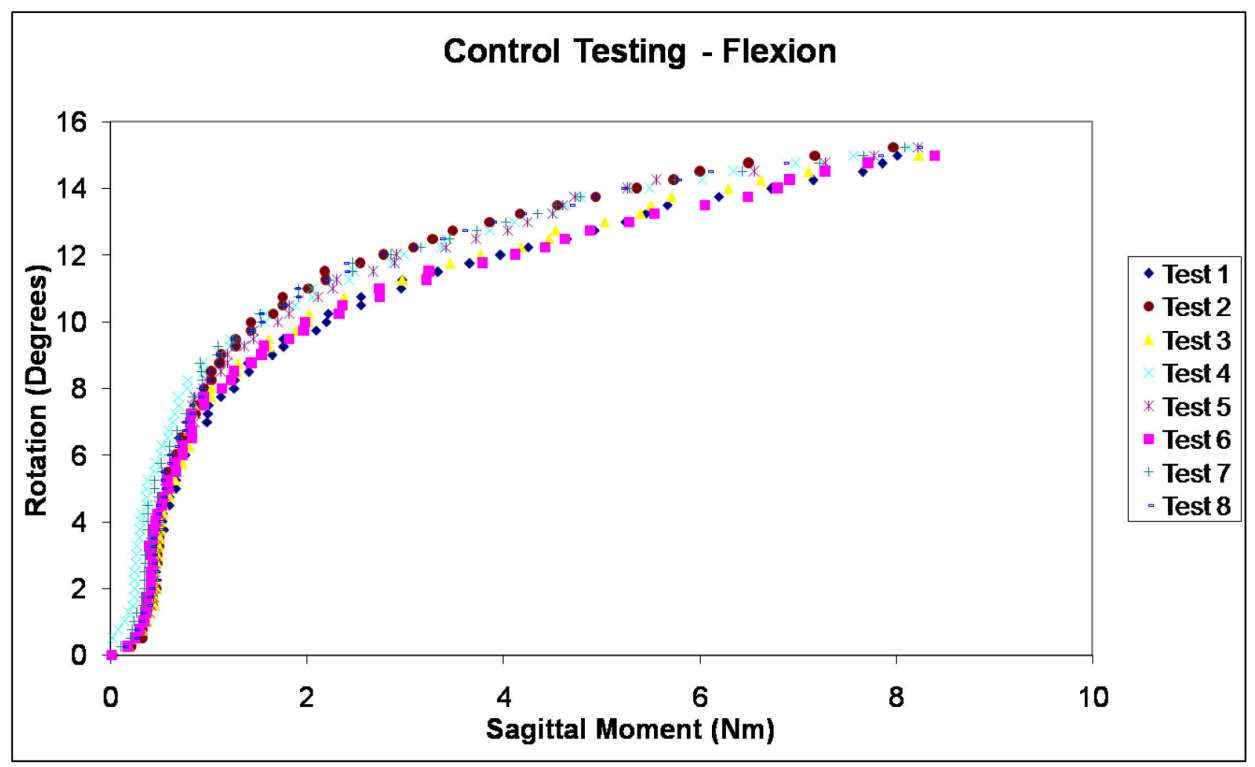

Figure 3-5 Control Specimen Graph. Graph of the resulting flexibility curve of the control specimen tested eight times in flexion. The graph is for the loading portion of the motion only.

Table 3-2 Rotation (Degrees) during Four Modes of Bending.

\begin{tabular}{ccc}
\hline Mode of Bending & Mean & S.D. \\
\hline Flexion & 8.05 & 2.96 \\
Extension & 5.46 & 1.90 \\
Left Lateral & 5.18 & 2.01 \\
Right Lateral & 5.43 & 1.94 \\
\hline
\end{tabular}




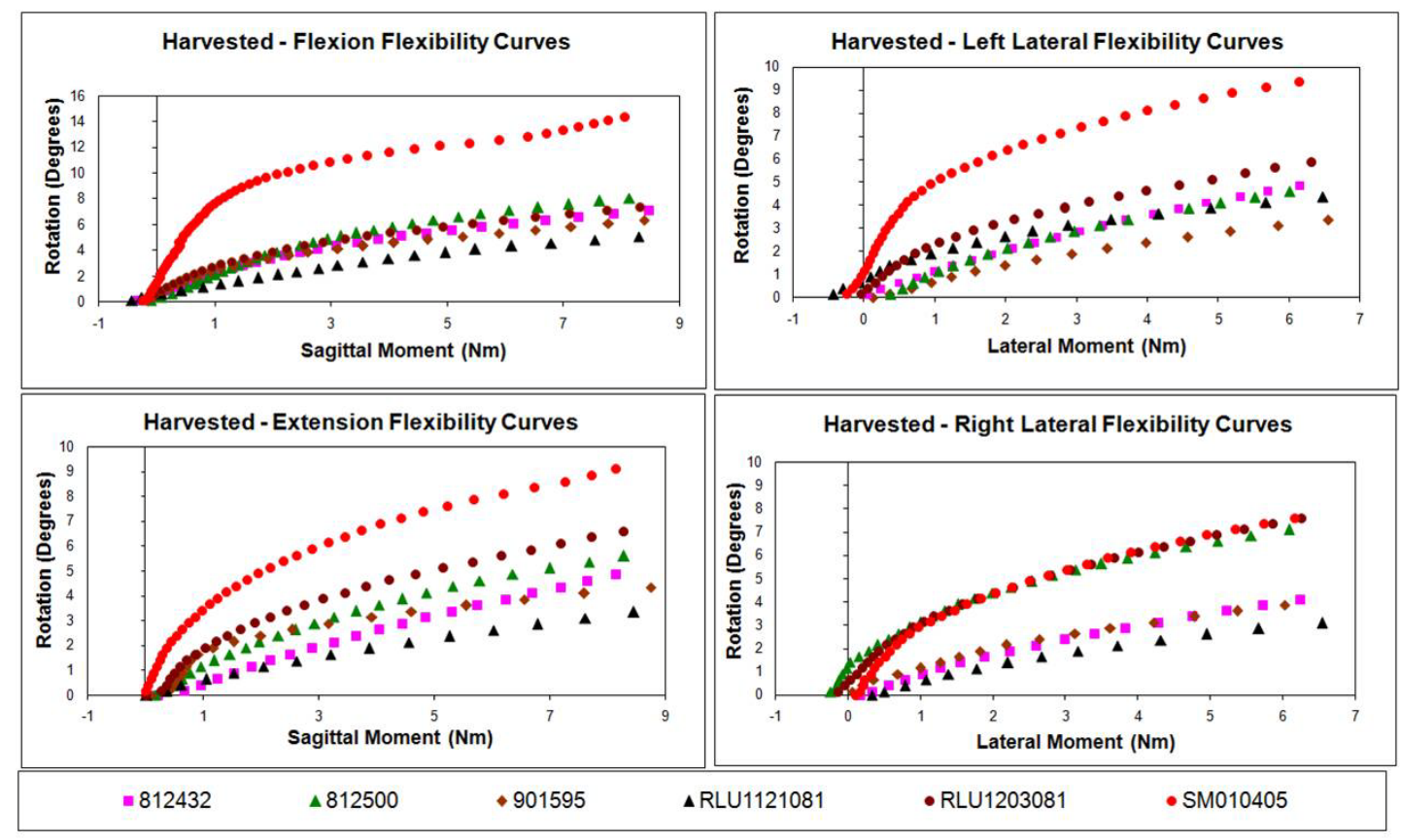

Figure 3-6 Specimen Flexibility Curves. Curves for each specimen during the four modes of bending.

Table 3-3 Average Forces (N) along the Harvested Quasistatic Path.

\begin{tabular}{ccc}
\hline Mode of Bending - Force & Mean & S.D. \\
\hline Flexion - Shear Force & -0.34 & 1.08 \\
Flexion - Axial Force & -0.49 & 1.13 \\
Extension - Shear Force & -0.06 & 0.95 \\
Extension - Axial Force & -0.54 & 1.12 \\
Left Lateral - Shear Force & -0.46 & 0.77 \\
Left Lateral - Axial Force & 0.23 & 1.10 \\
Right Lateral - Shear Force & -0.28 & 0.87 \\
Right Lateral - Axial Force & 0.10 & 1.18 \\
\hline
\end{tabular}



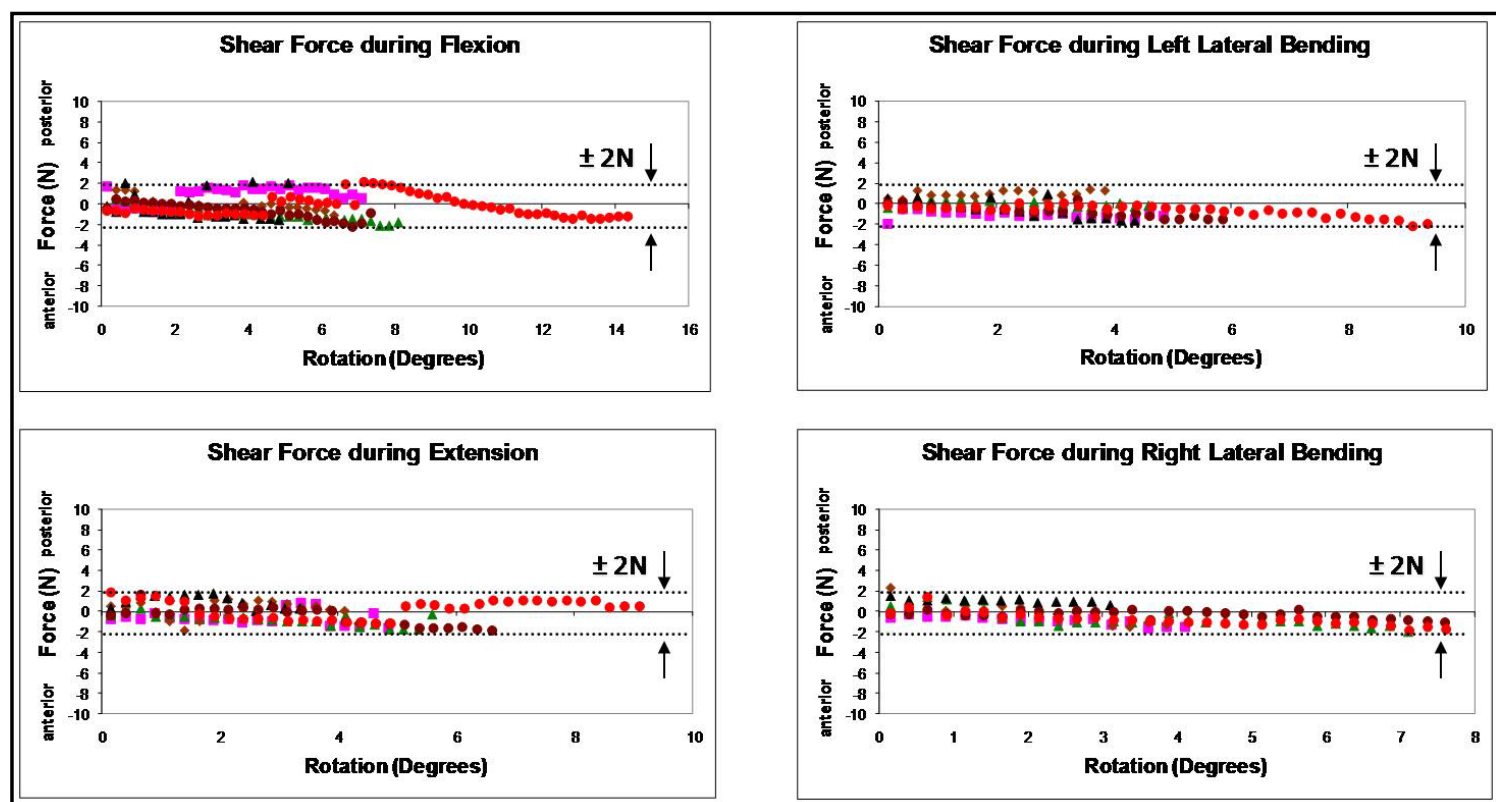

$=812432 \Delta 812500 \bullet 901595 \Delta$ RLU1121081 •RLU1203081 • SM010405

Figure 3-7 Shear Forces for Each Specimen during Four Modes of Bending.
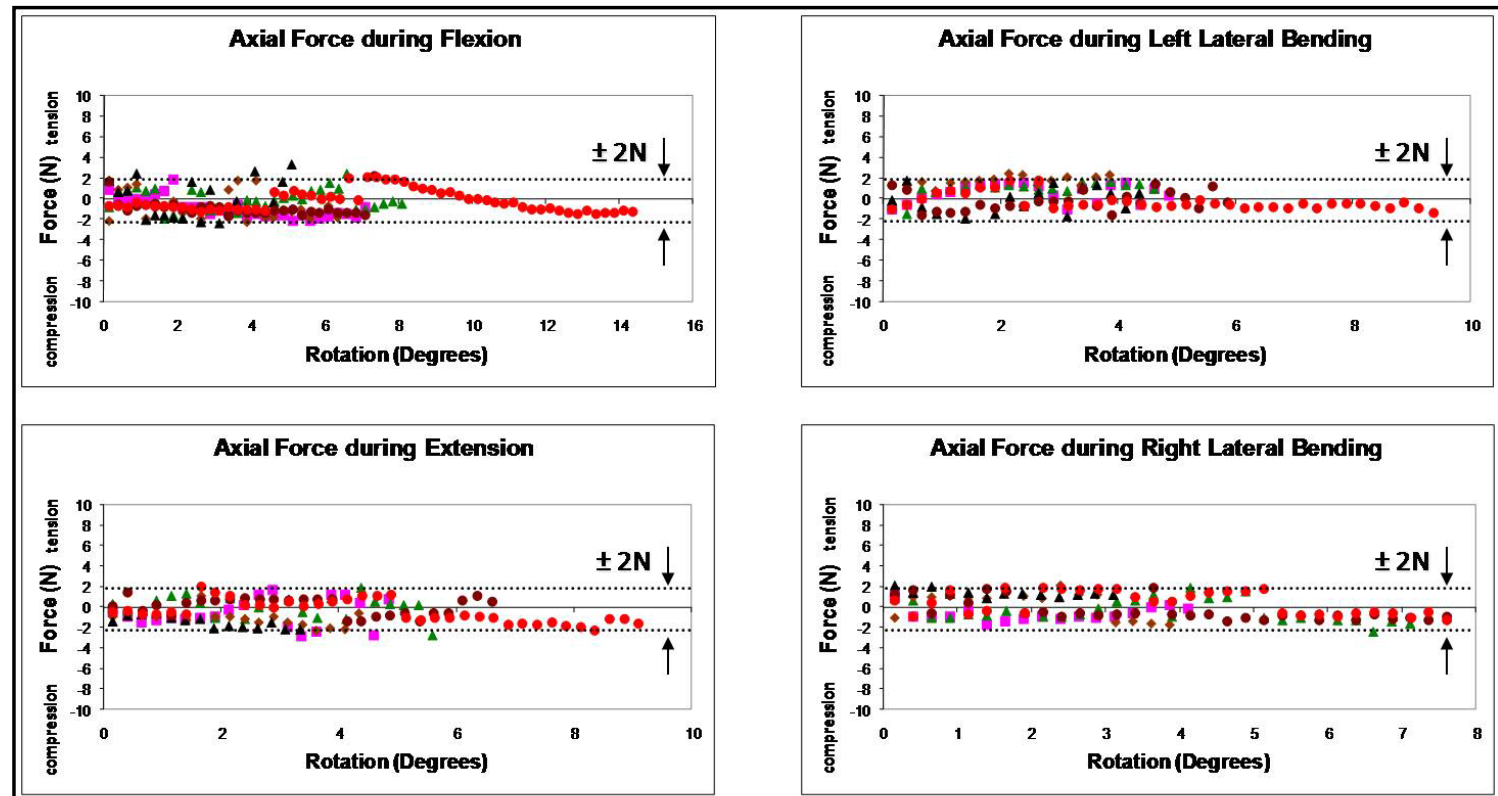

- $812432 \triangle 812500 \bullet 901595 \Delta$ RLU1121081 •RLU1203081 • SM010405

Figure 3-8 Axial Forces for Each Specimen during Four Modes of Bending. 
rotation for the four modes of bending. Sign conventions for these graphs are the same as what was noted for Table 3-3.

For all specimens and all modes of bending, both the shear and axial forces stayed within the $\pm 2 \mathrm{~N}$ range that was specified by the user. The total average shear and axial forces along the quasistatic paths were $0.95 \mathrm{~N}$ and $1.18 \mathrm{~N}$, respectively. The total force average was $1.51 \mathrm{~N}$ and the average translation of the center of rotation throughout testing was $0.062 \mathrm{~mm}$. Multiplying these two average values gives the average moment contributed to the specimen bending moment: $0.000094 \mathrm{Nm}$. This value represents only $0.0012 \%$ of the total bending moment for flexion/extension testing or $0.0016 \%$ of the total bending moment for left and right lateral bending testing.

IAR values for the harvested specimens can be seen in Figure 3-9. Each zone (yellow for flexion and red for extension) represents the average and standard deviations zones where the IAR was located during each test. Calculated IAR values were normalized to the height and width of the harvested disc space of each specimen as measured from radiographs. Average normalized IAR locations can be seen overlaid over a representative lumbar MSU for the harvested condition in Figure 3-10. A statistically significant difference was seen between the $\mathrm{X}$-coordinate locations of the IAR, but not the Y-coordinates.

\subsection{Concluding Discussion}

Members of the Biomechanics Laboratory at The UTHSC had a strong desire to replicate the pure moment protocol because it is such a widely used testing methodology. This study demonstrated the ability of the Spine Robot to find a quasistatic path of minimal loading for a single-level lumbar MSU that equated to a pure moment loading scenario. The resulting rotations in flexion/extension and left/right lateral bending are agreeable with previously published studies testing harvested L4-L5 segments. A comparison between this study and other in-vitro and in-vivo studies can be seen in Figure 3-11 and Figure 3-12, respectively. For the in-vitro study, all testing was under pure moment conditions to the moment limits stated in the legend. If a preload axial force compressed the specimen prior to testing, this is also mentioned.

As stated previously, the average moment error associated with testing only represented $0.0012 \%$ of the total moment during flexion/extension and $0.0016 \%$ during lateral bending. The maximum force error during testing was found to be $2.56 \mathrm{~N}$ and the maximum moment arm was found to be $0.599 \mathrm{~mm}$. This means that the even during the worst off-axis loading scenario experienced in the study, the system was still $99.98 \%$ accurate.

In a study conducted by Eguizabal et al, the cable-driven pure moment method was tested in two configurations: the common "fixed-ring" configuration developed by Crawford in $1995^{10}$ and the novel "sliding-ring" configuration. In the "fixed-ring" setup, the tested specimen underwent a shear load between 10.3 and $15.7 \mathrm{~N}$, equating to between 


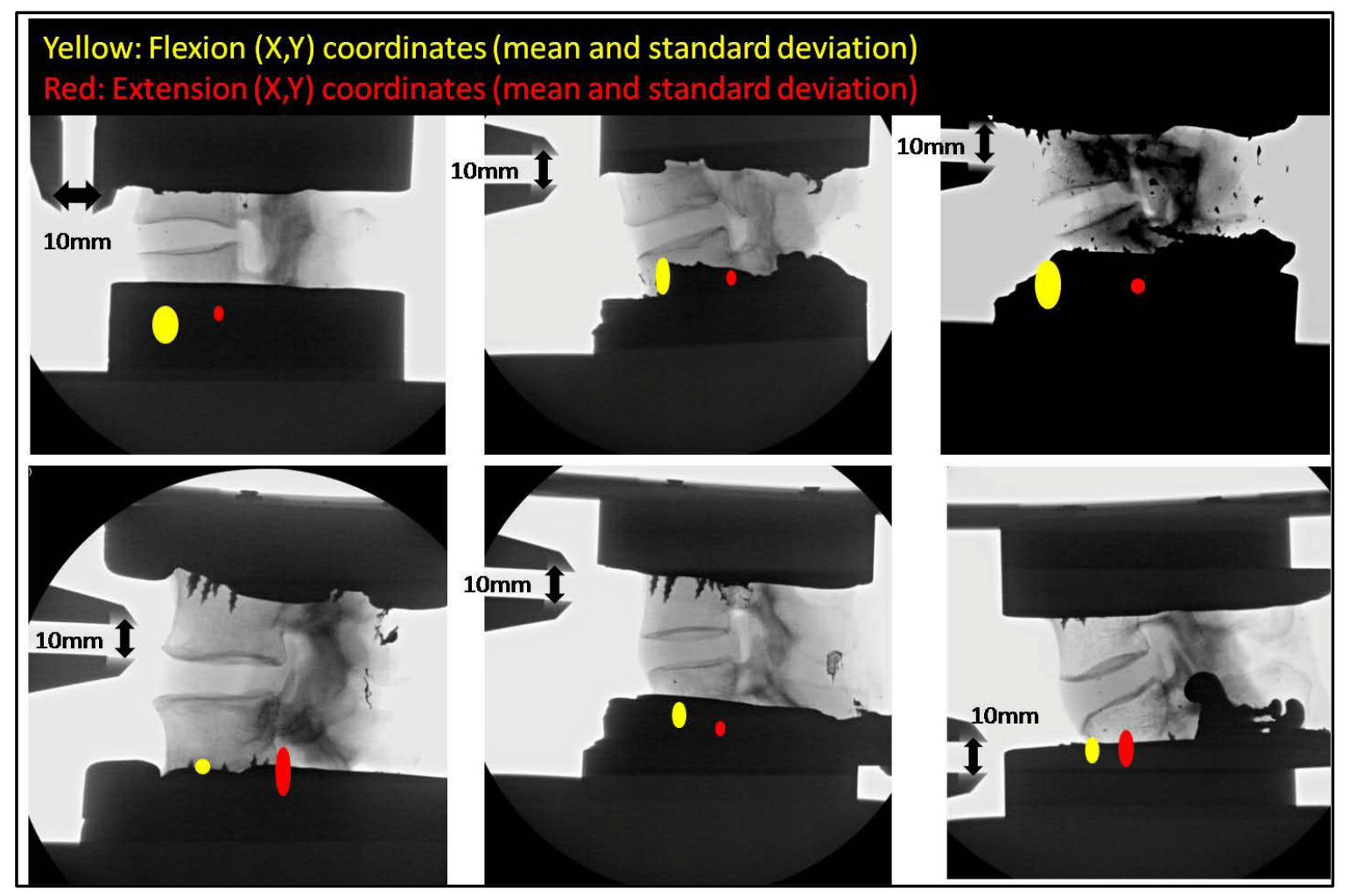

Figure 3-9 Calculated IAR Locations on Radiographs. The yellow zones represent the average and standard deviations of the flexion locations, while the red zones represent the average and standard deviations of the extension locations. The calipers in each image are set to $10 \mathrm{~mm}$. 


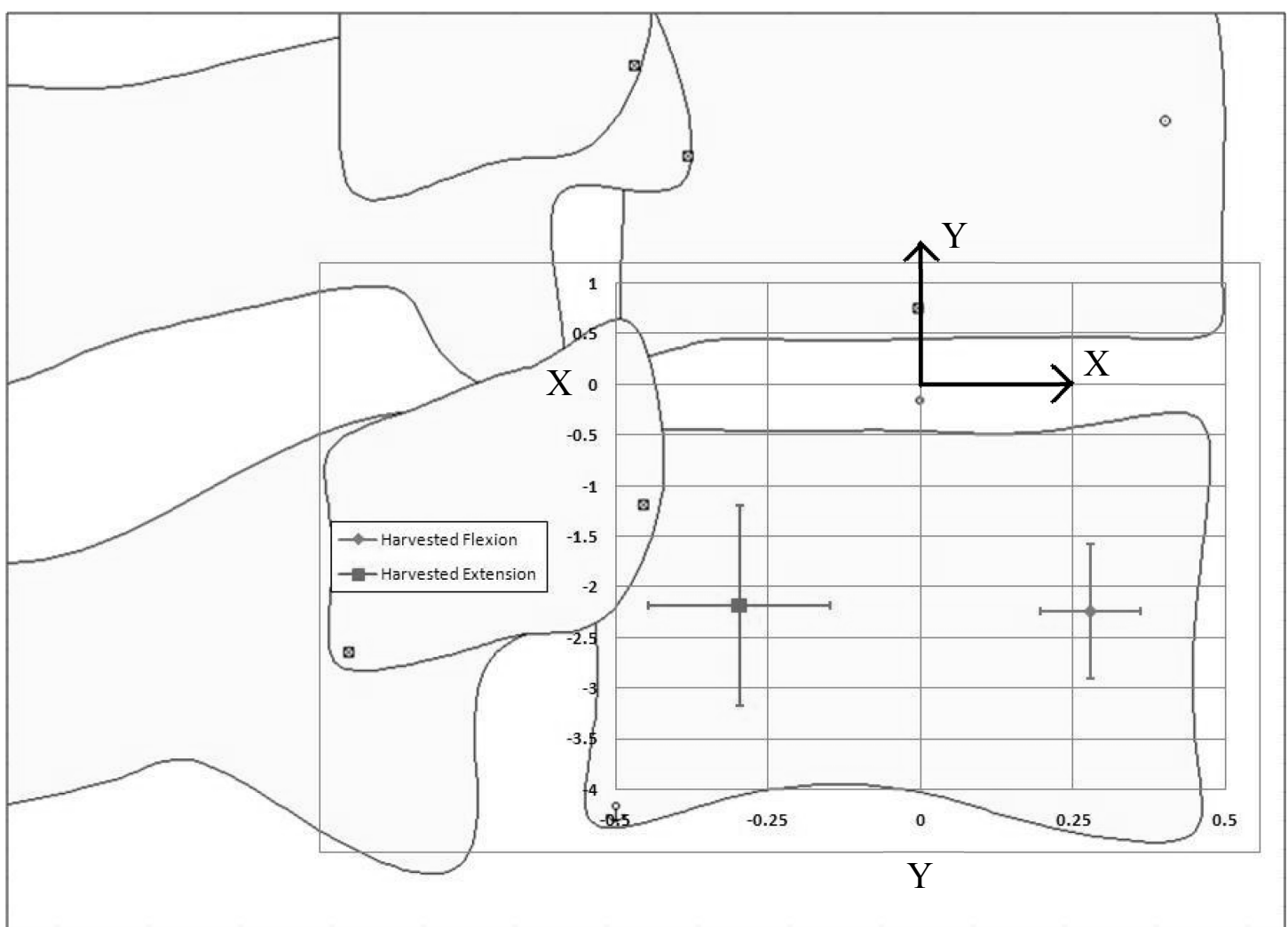

Figure 3-10 Normalized IAR Locations. Representative lumbar overlaid with a graph of the normalized harvested IAR locations (average with error bars). 1 unit on the x-axis is equivalent to the sagittal length of the cranial vertebral body. 1 unit on the y-axis is equivalent to harvested disc height. 


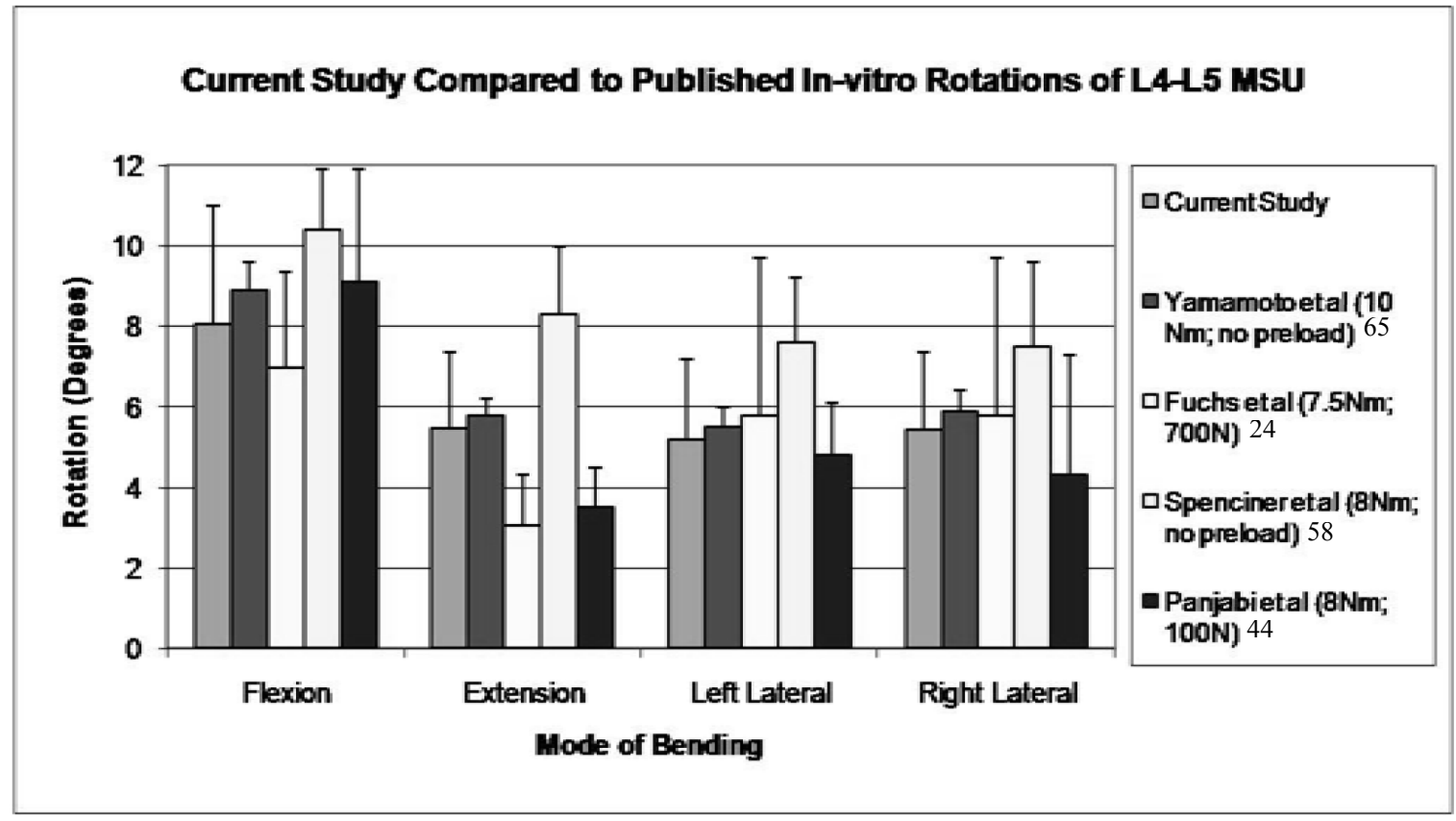

Figure 3-11 Comparison to Published In-vitro Rotations. Comparison between current study and published values for L4-L5 rotations from other in-vitro studies.

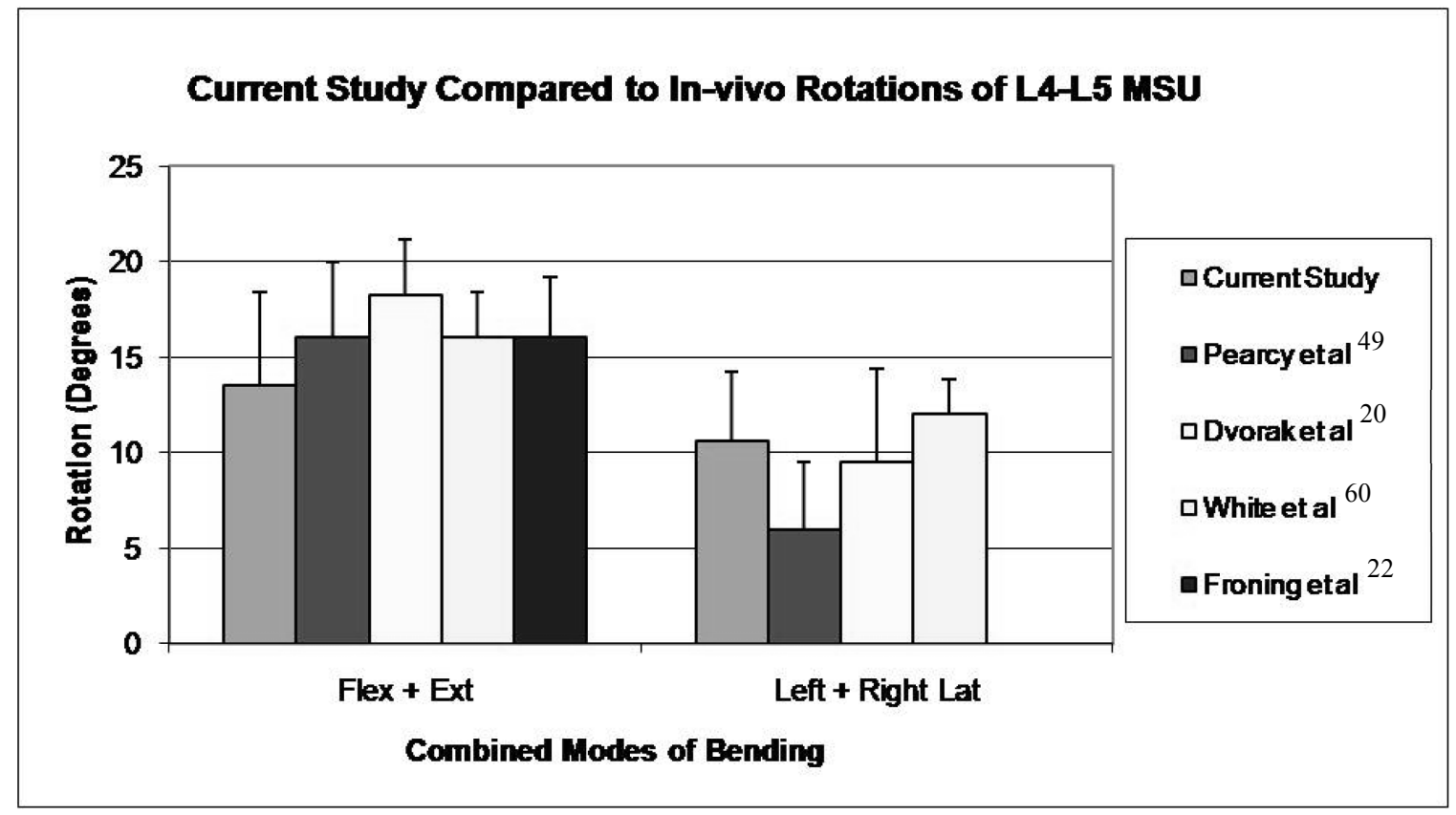

Figure 3-12 Comparison to Published In-vivo Rotations. Comparison between current study and published values for L4-L5 rotation from in-vivo studies. 
8.2 and $12.7 \%$ of the total moment. The "sliding-ring" configuration yielded shear force results between 3.6 and $6.6 \mathrm{~N}$, or between 2.9 and $5.6 \%$ of the total moment. The pure moment method presented in this paper yields force errors that are at worst two orders of magnitude lower than either the common cable-driven method of inducing pure moments or the modified test setup designed to improve this method. ${ }^{21}$ A comparison of the current study to the study conducted by Eguizabal et al can be seen in Table 3-4.

The calculated values of the IAR locations for the harvested specimen showed a statistically significant difference in the A-P location between flexion and extension test. This is in disagreement with many previous studies where the flexion and extension values were shown near the disc center, which is almost directly between the separate A$P$ points found in this study. Considering the potential errors associated with both in-vivo ad in-vitro IAR calculations discussed previously, it would be expected that these values would average to a region near the center of the disc. The locations found in this study come from measurements with a high degree of accuracy based on the high resolution of the Spine Robot's axes.

Future modifications to our pure moment protocol include the introduction of a compressive load that simulates the follower load idea or replication of a net muscle load vector. It is also a desire of the lab to use the data provided by the Spine Robot to calculate instantaneous axes of rotation for specimen throughout the quasistatic pathfinding process. Pure moment testing comparing harvested to implanted specimen is planned.

Although pure moment testing methods are acceptable for evaluating fusion spinal instrumentation, limitations such as the lack of a shear load and introduction of non-physiological motion response exist. Additionally, it is unlikely that this methodology is an appropriate load-control protocol for testing non-fusion instrumentation such as disc arthroplasty devices. The Spine Robot has already been used for complex kinematic testing such as fixed-axis testing. We plan to develop more advanced techniques such as coupled loading scenarios and the introduction of physiologic shear loads to better replicate anatomical conditions experienced in-vivo. The goal of all biomechanical testing should be to replicate as close as possible the natural movements of the human body. We believe the programmable Spine Robot allows us to get closer to this goal than previous testing apparatus. 
Table 3-4 Off-axis Loading in Current Study Compared to Common Pure Moment Methods.

\begin{tabular}{ccccc}
\hline Method & Force Error $(\mathrm{N})$ & Moment Arm (mm) & Moment Error (Nm) & \% Error of Max. Bending Moment \\
\hline Current Protocol & $0.09-2.56$ & $0.008-0.599$ & $7.2 * 10^{-7}-0.0015$ & $9.0 * 10^{-6}-0.019 \%(8 \mathrm{Nm}$ max.) \\
Fixed-Ring & $10.3-15.7$ & 36 (ring radius) & $0.37-0.57$ & $8.2-12.7 \%(4.5 \mathrm{Nm}$ max.) \\
Sliding-Ring & $3.6-6.6$ & 36 (ring radius) & $0.13-0.24$ & $2.9-5.6 \%(4.5 \mathrm{Nm}$ max. $)$ \\
\hline
\end{tabular}




\section{Chapter 4 - An In-vitro Comparison of Harvested Lumbar Segments to Those Implanted with a Constrained Total Disc Replacement Device under Pure Moment Loading}

\subsection{Introduction}

Degenerative Disc Disease (DDD) is a common clinical problem that affects up to $96 \%$ of Americans over 60 in their lifetime. ${ }^{37}$ Between the therapeutic cost of intervention to treat DDD and the loss of productivity for those afflicted with the disease, it is estimated that DDD leads to an annual cost of $\$ 50$ billion. ${ }^{23}$ The first options for care generally involve medicinal pain relief and different forms of physical therapy. For patients for whom surgical intervention is recommended, spinal fusion has been the standard treatment for clinicians for over 50 years. Spinal fusion reduces pain by severely limiting motion at the fused joint and also restores proper spacing between vertebral bodies. Although this procedure is common, recently its efficacy in pain management has been called into question as it has the potential to cause adjacent level effects on nearby spinal MSUs. ${ }^{47}$

For decades, researchers have searched for alternatives to spinal fusion. Ideally, an alternative would restore proper disc height much like fusion surgery but also restore normal biomechanical properties to the diseased $\mathrm{MSU}{ }^{3}$ Recently, biomedical companies have begun to manufacture total disc replacement (TDR) devices to treat DDD in the place of fusion. TDR devices fall into two categories: unconstrained and constrained. The unconstrained TDR has a mobile core that allows both translation and rotation of the implanted MSU. Unconstrained TDRs allow for changes in the segments axis of rotation which helps to restore the original biomechanical properties of the spinal disc, but can cause to increased shear loading on the posterior spinal structures. A constrained TDR contains a ball-and-socket articulation that allows rotation along a fixed center of rotation. The constrained TDR does not allow any translation or the changing of the segment's center of rotation, but it does prevent excessive shear loading on the posterior structures of the segment. ${ }^{3,29}$

Biomechanics testing is used to compare the physiological characteristics of the human spine to those induced by medical devices. A common method of load-controlled mechanical testing of human cadaveric spines is the pure moment protocol. The pure moment testing method involves the application of a pure rotational load to a free and unsupported end of spinal segment. The opposite end of the spinal segment is held stationary via fixation to the base of the testing platform. If the top of the spinal segment is supported by the testing apparatus, then a pure moment can be applied to the spine by minimizing the axial and shear forces that the segment experiences. ${ }^{27,42}$

The purpose of this research was an in-vitro comparison between human cadaveric lumbar MSUs and those implanted with the ProDisc-L device. The test protocol involved subjecting the individual MSUs to pure bending moments in flexion/extension and left and right lateral bending utilizing the Spine Robot at UTHSC. 
The development of the custom-written pure moment protocol is outlined in Chapter 3. Motion characteristics of the harvested and implanted specimen were recorded that allowed for the calculation of each MSUs instantaneous axis of rotation (IAR) during flexion/extension testing.

As previously discussed, the IAR for spinal segments is a characteristic that is not well defines in the literature. The constrained nature of the ball-and-socket TDR means that the specimen should rotate about a fixed point. It is critical to have an understanding of how this will affect spinal motion. Additionally, due to the nature of the constrained devices and their implantation procedure, the IAR location during extension motions is expected to change relative to flexion motions. ${ }^{29}$

\subsection{Materials and Methods}

Six fresh human cadaveric lumbar spines were procured from the Medical Education and Research Institute (Memphis, TN). All specimens were screened with anterior/posterior and lateral radiographs to exclude those with marked degenerative changes or significant osteoporosis and none of the donors died of diseases of or injuries to the spine. Bone density measurements were not done. The average age of the specimens was $59.7 \pm 11.7$ years. Specimens were frozen immediately after harvest and were thawed for 24 hours prior to disarticulation. Once thawed, each specimen was dissected into L4-L5 single-level MSUs with special care taken to preserve the disc, vertebral bodies, posterior bony surfaces, and interconnecting ligaments. Once the specimens were disarticulated, the endplates of each vertebral body were cleaned and screws were inserted into them for retention during the potting phase. MSUs were potted in a bismuth alloy (Small Parts, Miami Lakes, FL). Utilizing the radiographs made during the screening phase, the disc angles of the L4-L5 disc were measured relative to the cranial endplate of $\mathrm{L} 1$. These angles were maintained while potting the specimen. Specimens were potted with the top and bottom bismuth plates parallel to allow for a repeatable mounting procedure. Finally, potted specimens were radiographed to ensure that the disc angle was preserved and that no damage occurred during potting. A potted specimen can be seen in Figure 4-1.

The MSUs were tested under two conditions: harvested and implanted. For the implanted condition, the constrained ProDisc-L from Synthes Spine (Synthes Inc., West Chester, PA) was chosen. The ProDisc-L has two cobalt chromium endplates with titanium plasma-sprayed surfaces and a high molecular weight polyethylene core. The cranial endplate contains the polished socket for the ball-and-socket articulation, while the polyethylene core contains the $15 \mathrm{~mm}$ radius ball portion. The ProDisc-L is an example of a constrained TDR. ${ }^{4}$ An assembled ProDisc-L can be seen in Figure 4-2.

Harvested testing required no further modification to the tissue after potting. After harvested testing was completed, the MSUs were implanted with the ProDisc-L TDR device. A spine surgeon performed an anterior discectomy under radiography from a fluoroscopic C-arm (GE 9600 or 9800, GE Healthcare, Chalfont St. Giles, United 


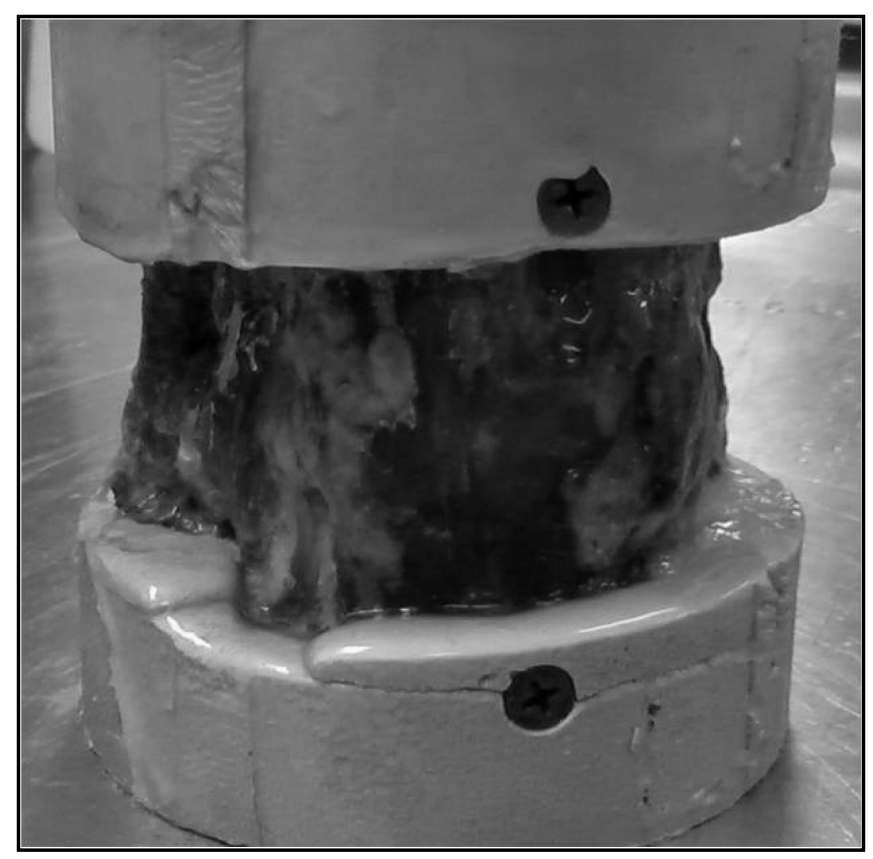

Figure 4-1 Potted L4-L5 MSU. The top and bottom plates are kept parallel to ensure repeatable mounting of the specimen.

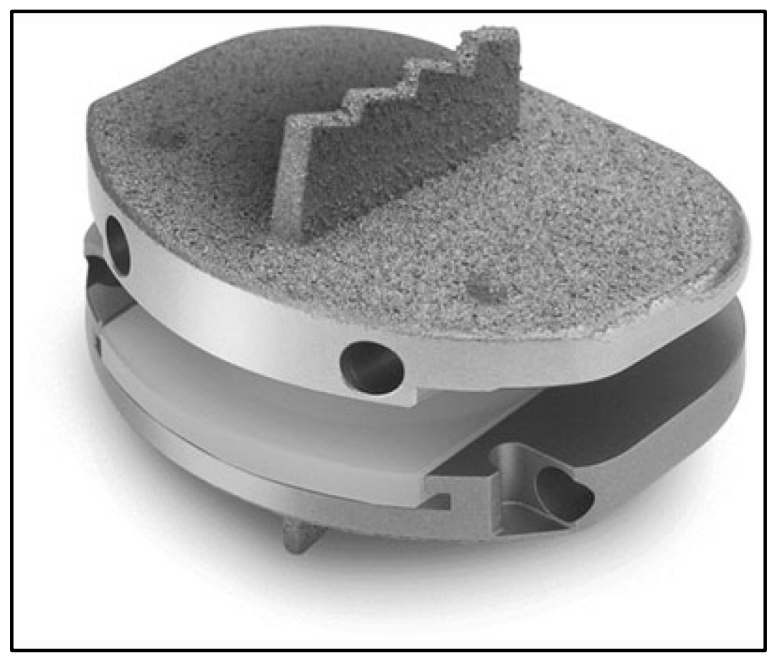

Figure 4-2 Assembled ProDisc-L (Synthes Spine) Total Disc Arthroplasty Device. The implant contains two cobalt-chromium endplates with titanium plasma-sprayed bone-interfacing surfaces and a high-molecular weight polyethylene core. Reprinted with permission from Zigler J MD, Delamarter R MD, Spivak JM MD, et al. Results of the prospective, randomized, multicenter food and drug administration investigational device exemption study of the prodisc-1 total disc replacement versus circumferential fusion for the treatment of 1-level degenerative disc disease. Spine 2007;32:1155-1162. ${ }^{67}$ 
Kingdom). After discectomy, the spine surgeon implanted the ProDisc-L devices using the guidelines outlined in the Technique Guide (Synthes Spine). Care was taken to place the implant properly with respect to both lateral and sagittal balance. Images from surgery and harvested vs. implanted radiographs can be seen in Figure 4-3 and Figure $4-4$, respectively. The increase in lordotic angle of the specimens after ProDisc-L implantation averaged $5.79^{\circ} \pm 2.83$.

All testing was performed utilizing the custom-written pure moment protocol for the UTHSC Spine Robot described in Chapter 3. The Spine Robot and its four degrees of freedom can be seen in Figure 4-5. The Spine Robot rotated each specimen under different modes of bending in 0.25 degree increments, using small translations to offset resulting axial and shear forces to load the specimen under pure bending moments. Once the testing was complete, a quasi-static path of minimal loading was generated and all force and moment values experienced by the specimen along the path were recorded. For flexion/extension tests, specimens were rotated to an $8 \mathrm{Nm}$ limit. For lateral bending tests, the specimens were rotated to $6 \mathrm{Nm}$ and were unconstrained in axial rotation. Path finding took approximately 4-5 minutes per mode of bending test. Range of motion data was compared between the harvested and implanted group with a one-way ANOVA $(\mathrm{p}<0.05)$. Throughout all testing, force and moment values that were not being investigated were monitored and recorded and were not significant.

In order to calculate the IAR for each specimen, equations derived by Crisco et $\mathrm{al}^{12}$ were used. These equations use perpendicular bisectors of two vertebral points over time. Figure 4-6 shows the motion of the two points and relative position vectors. If the two points over time are labeled as A1(x1,y1), A2(x2,y2), B1(x3,y3), and B2(x4,y4), then the position vectors $\mathbf{u} \mathbf{1}$ and $\mathbf{u} \mathbf{2}$ equal:

$$
\begin{aligned}
& \mathbf{u} \mathbf{1}=(A 1 x-B 1 x) i+(A 1 y-B 1 y) j \\
& \mathbf{u} \mathbf{2}=(A 2 x-B 2 x) i+(A 2 y-B 2 y) j
\end{aligned}
$$

and the cosine of the angle between the position vectors is:

$$
\cos \phi=\frac{u 1 \cdot u 2}{|u 1||u 2|}
$$

For this research Phi $(\Phi)$ corresponds to the pure rotation angle the cranial vertebral body of each MSU between each incremental rotation along the quasistatic path. Because the Spine robot records the exact angle and position data of the cranial MSU, the IAR of each specimen can be calculated using the following equations:

$$
\mathrm{X}_{\mathrm{IAR}}=\frac{1}{2} \times(\mathrm{x} 1+\mathrm{x} 2)+\frac{(\mathrm{y} 1-\mathrm{y} 2) \times \sin \phi}{2 \times(1-\cos \phi)}
$$




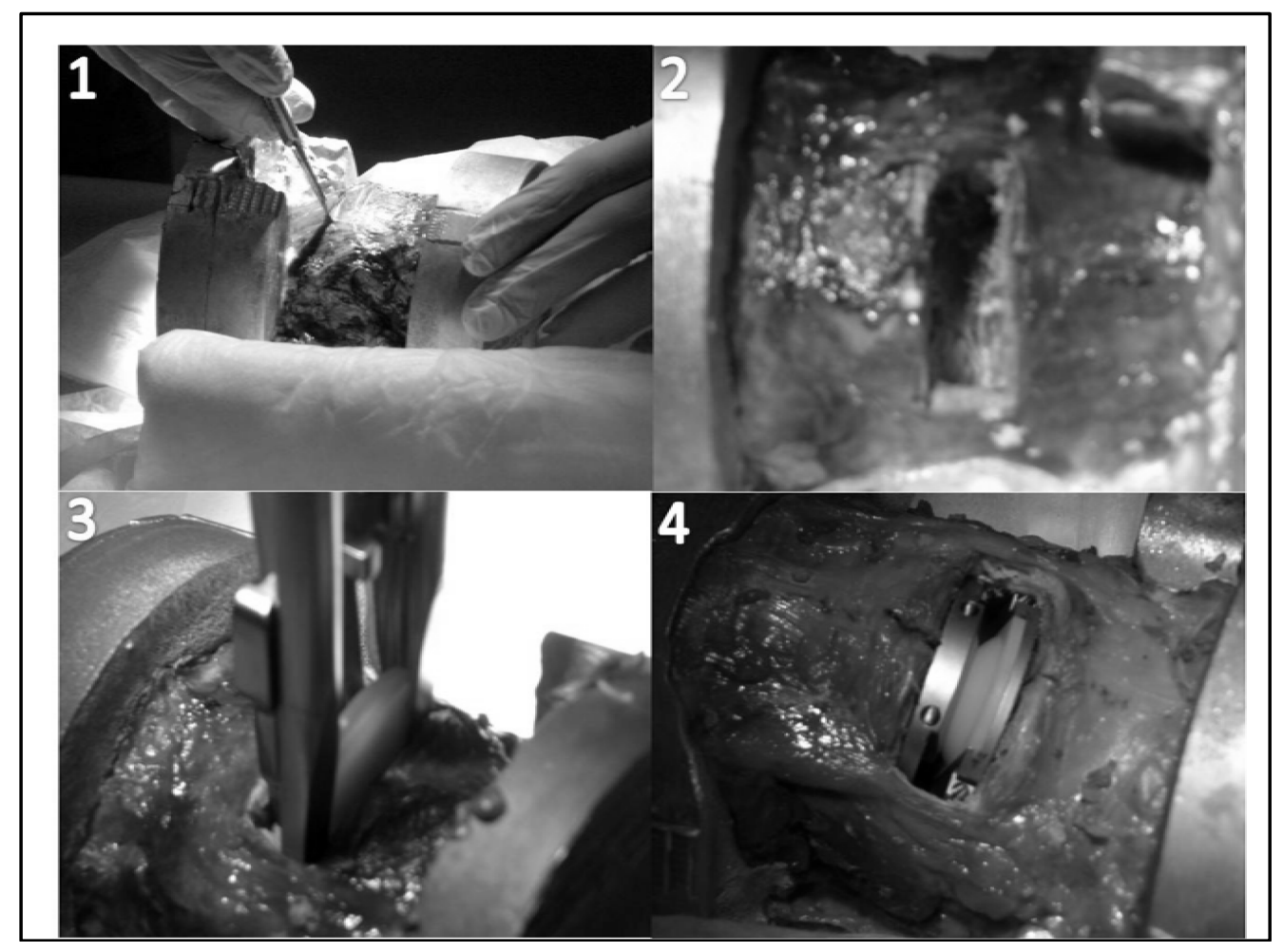

Figure 4-3 ProDisc-L Implantation. 1: Beginning the anterior discectomy procedure. 2: Complete discectomy of specimen. 3: Insertion of polyethylene core. 4: Completed ProDisc-L surgery.

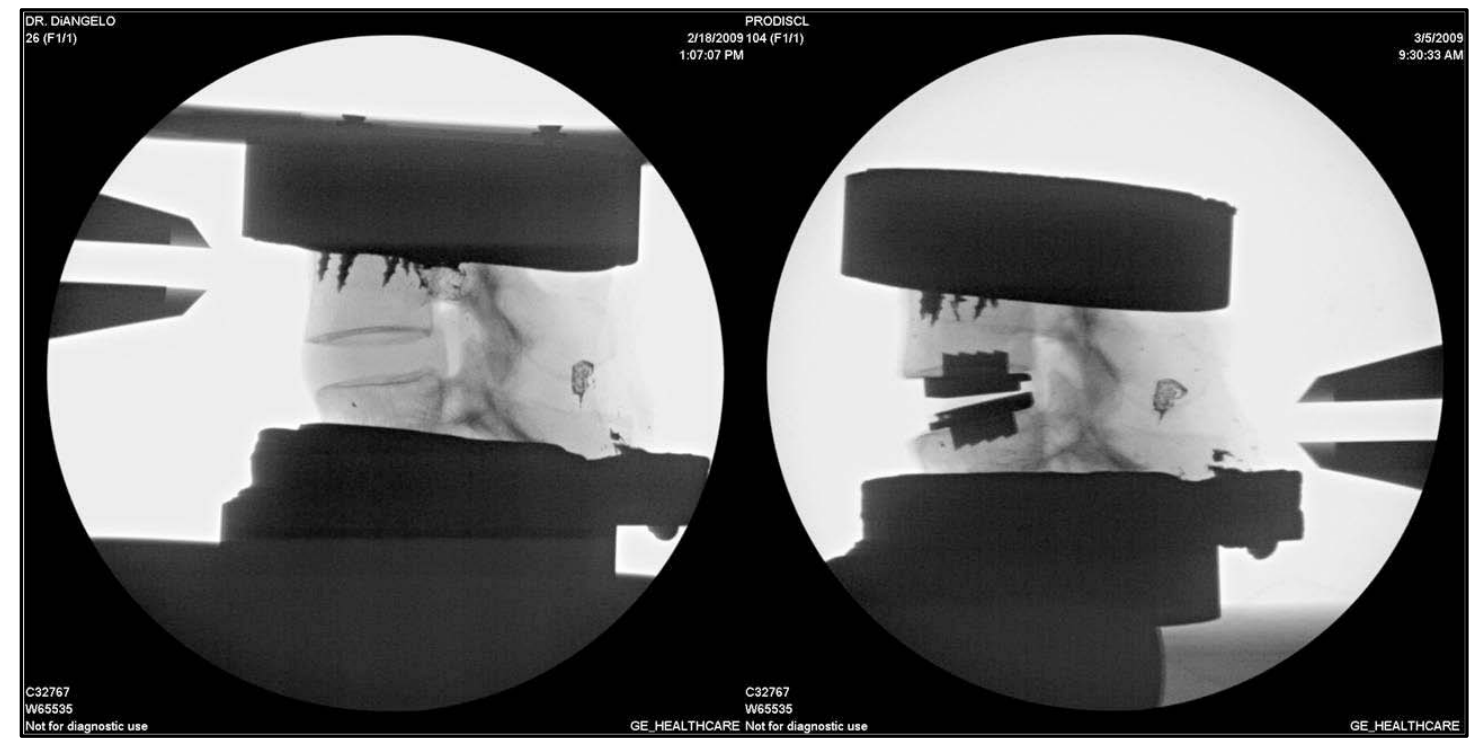

Figure 4-4 Lumbar MSU before and after ProDisc-L Implantation. 


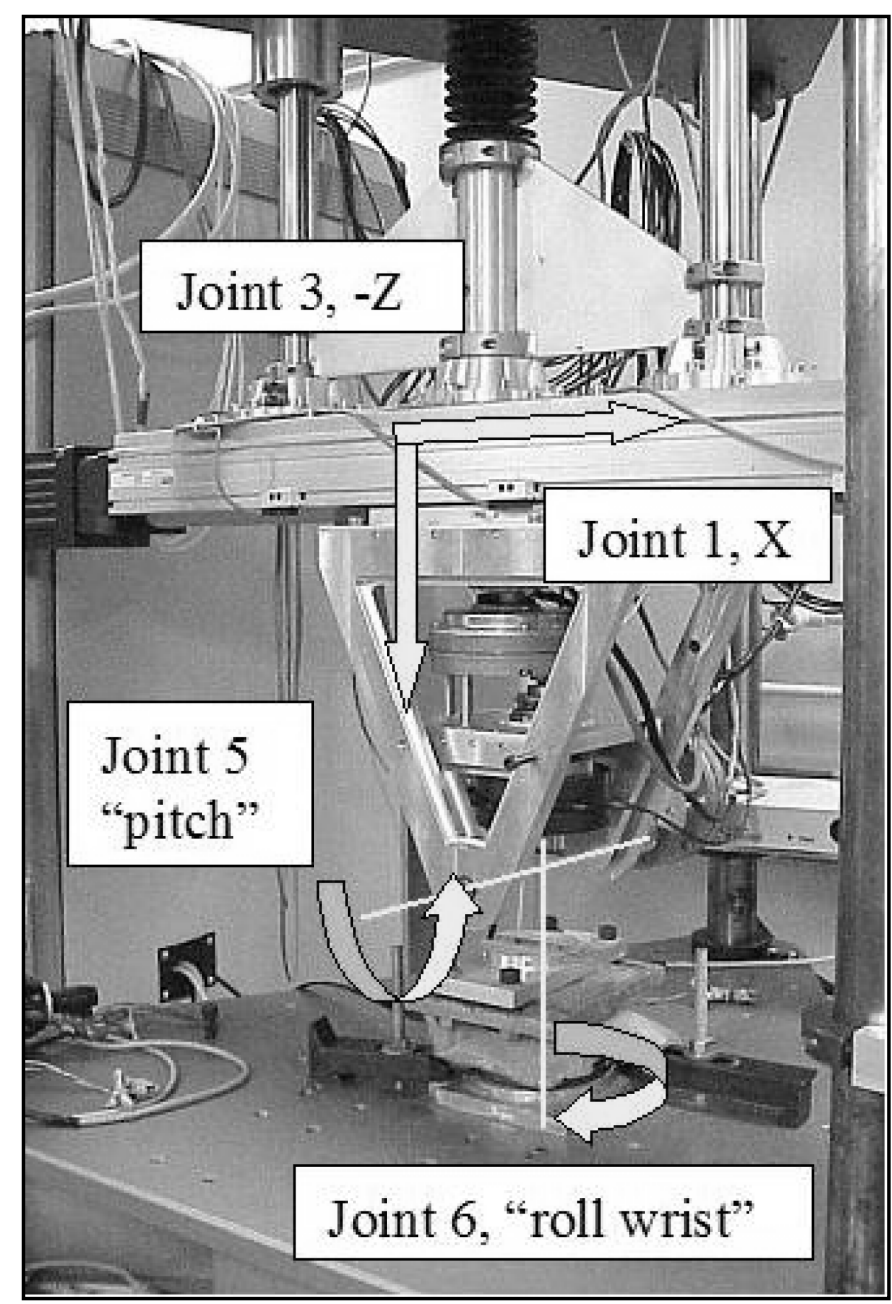

Figure 4-5 Spine Robot with Labeled Degrees of Freedom. Degrees of freedom are illustrated with arrows. A six axis load cell, located in the gimbal assembly, connects to the top mounting pot of the spinal segment. Reprinted with permission from Kelly BP. A multiaxis programmable spine robot for the study of multibody spinal biomechanics using real-time hybrid force and displacement control strategies. PhD Dissertation. The University of Tennessee Health Science Center, 2005. ${ }^{35}$ 


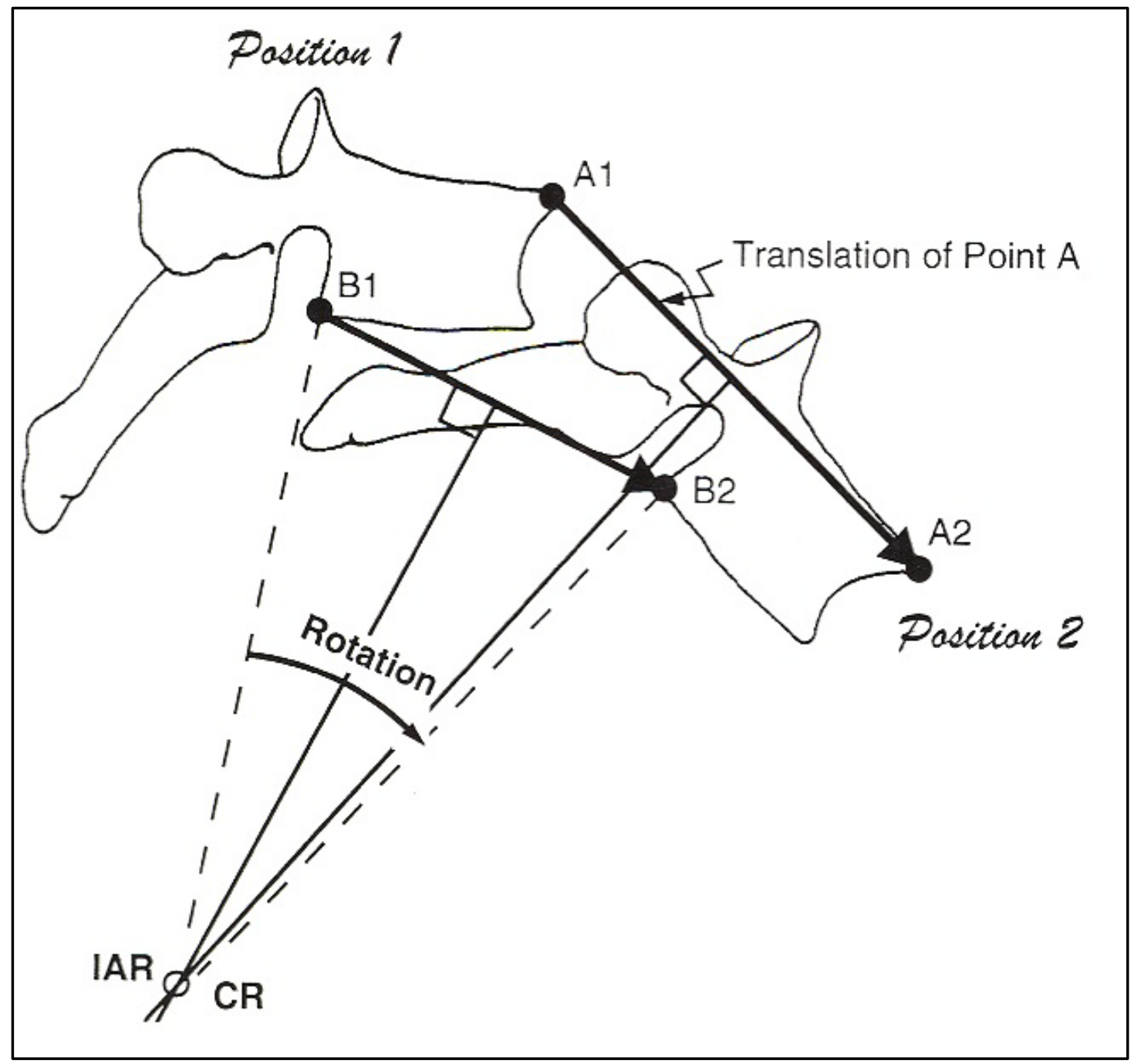

Figure 4-6 Calculation of the IAR. IAR is found using perpendicular bisectors of common vertebral body points between rotation intervals. Reprinted with permission from Clark CR, ed. The Cervical Spine. 3rd ed. Philadelphia: Lippincott-Raven Pub., $1998 .^{8}$ 


$$
\mathrm{Y}_{\mathrm{IAR}}=\frac{1}{2} \times(\mathrm{y} 1+\mathrm{y} 2)+\frac{(\mathrm{x} 1-\mathrm{x} 2) \times \sin \Phi}{2 \times(1-\cos \Phi)}
$$

Once the IAR values were calculated, the values were compared between modes of testing (flexion/extension) and specimen condition (harvested/implanted) with a oneway ANOVA with a Dunn's pair-wise multiple comparison test $(\mathrm{p}<0.05)$.

\subsection{Results}

Flexibility curves were generated for each specimen in each bending mode. These curves can be seen in Figure 4-7 and Figure 4-8 for the harvested and implanted conditions, respectively. The flexibility curves exhibited a pattern that is common for viscoelastic tissue testing. Range of motion graphs for individual and combined modes of bending are shown in Figure 4-9 and Figure 4-10, respectively. Significant differences between lateral modes of bending are indicated on the charts. The only significant differences found were in right lateral bending and combined lateral bending.

The average shear and axial forces found during specimen testing can be found in Table 4-1. A negative shear force represents an anterior shear for flexion/extension testing and a shear in the direction of rotation for lateral bending tests. A negative axial force represents a compressive force, while a positive axial force represents a tensile force.

After each incremental rotation the spine robot saved data for shear and axial forces experienced by the spine. Figure 4-11 illustrates the shear forces throughout rotation for the four modes of bending. Figure 4-12 illustrates the axial forces throughout rotation for the four modes of bending. Sign conventions for these graphs are the same as what was noted for Table 4-1. For all specimens and all modes of bending, all but a very small percentage of the shear and axial forces stayed within the $\pm 2 \mathrm{~N}$ range that was specified by the user. As a result, a pure moment loading condition was effectively replicated using the Spine Robot.

IAR values for harvested and implanted conditions can be seen in Figure 4-13 and Figure 4-14, respectively. Each zone (yellow for flexion and red for extension) represents the average and standard deviations zones where the IAR was located during each test. Calculated IAR values were normalized to the height and width of the harvested disc space of each specimen as measured from radiographs. Average normalized IAR locations can be seen overlaid over a representative lumbar MSU for the harvested and implanted conditions in Figure 4-15 and Figure 4-16, respectively. Significant differences between the $\mathrm{x}$ - and $\mathrm{y}$-locations between bending modes and specimen conditions can be seen in Table 4-2. 


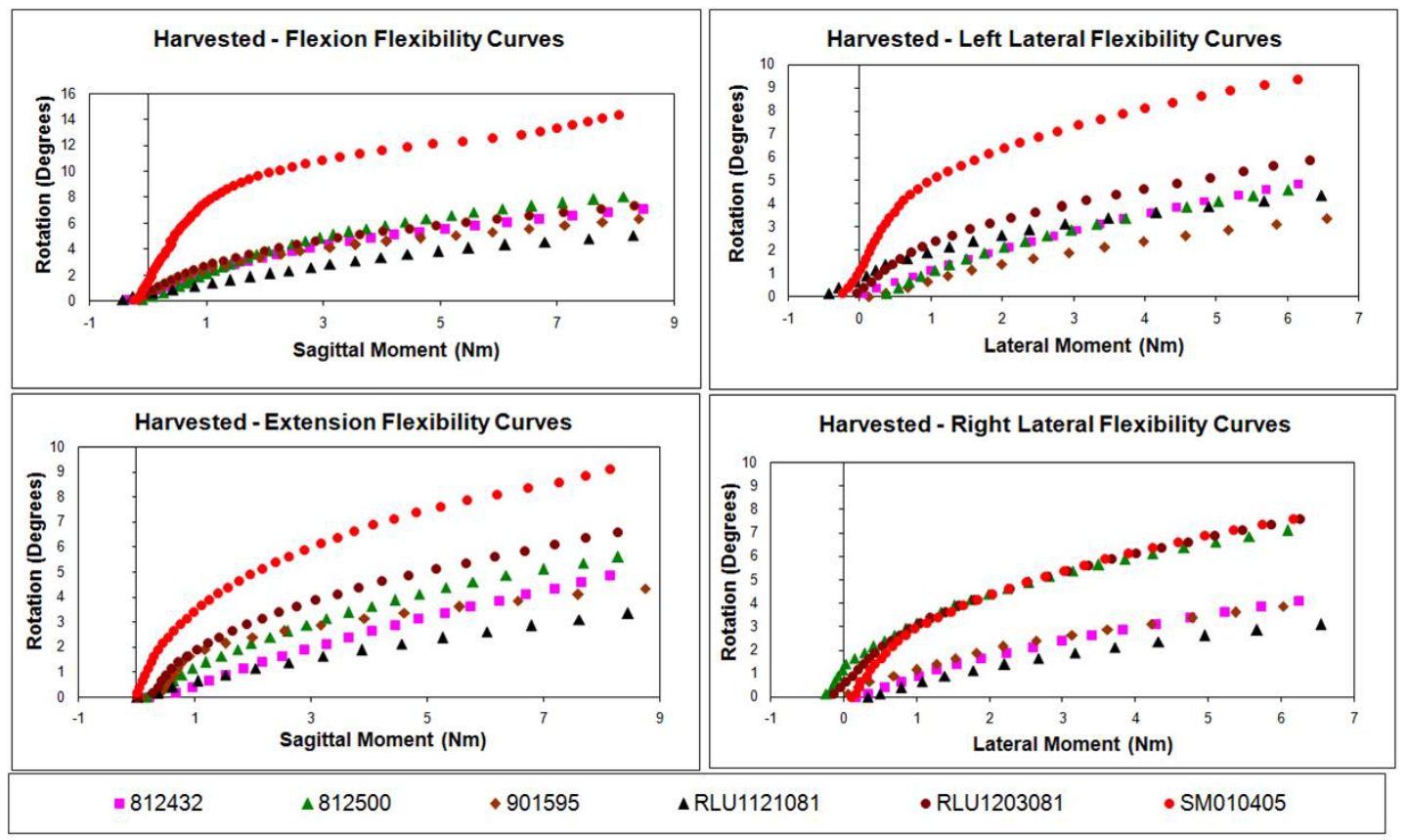

Figure 4-7 Harvested Flexibility Curves. Curves are shown for each specimen during the four modes of bending.
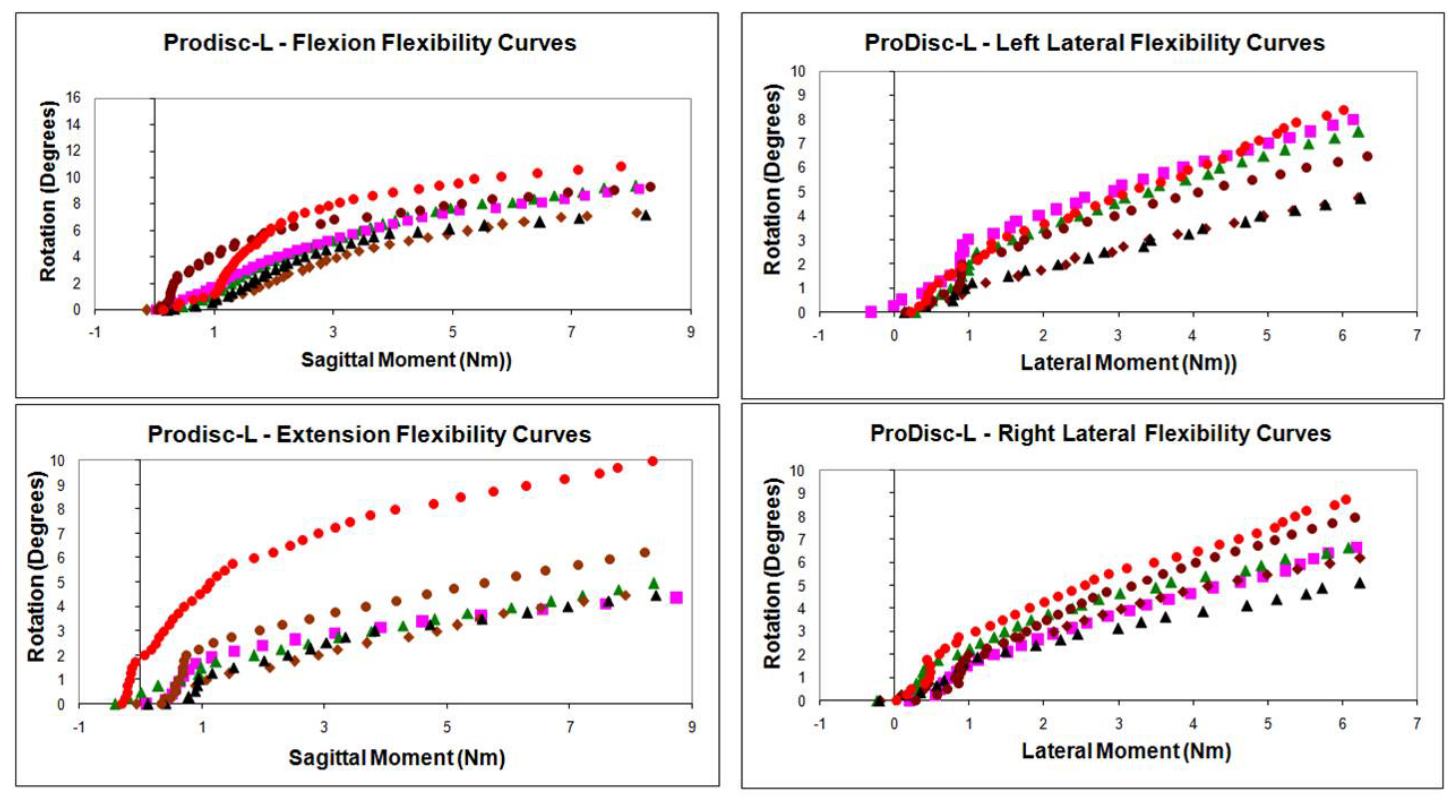

\begin{tabular}{|llllll|}
\hline $\mathbf{8 1 2 4 3 2}$ & $\mathbf{\Delta 8 1 2 5 0 0}$ & $\bullet 901595$ & ARLU1121081 & $\bullet$ RLU1203081 & $\bullet$ SM010405 \\
\hline
\end{tabular}

Figure 4-8 Implanted Flexibility Curves. Curves are shown for each specimen during the four modes of bending. 


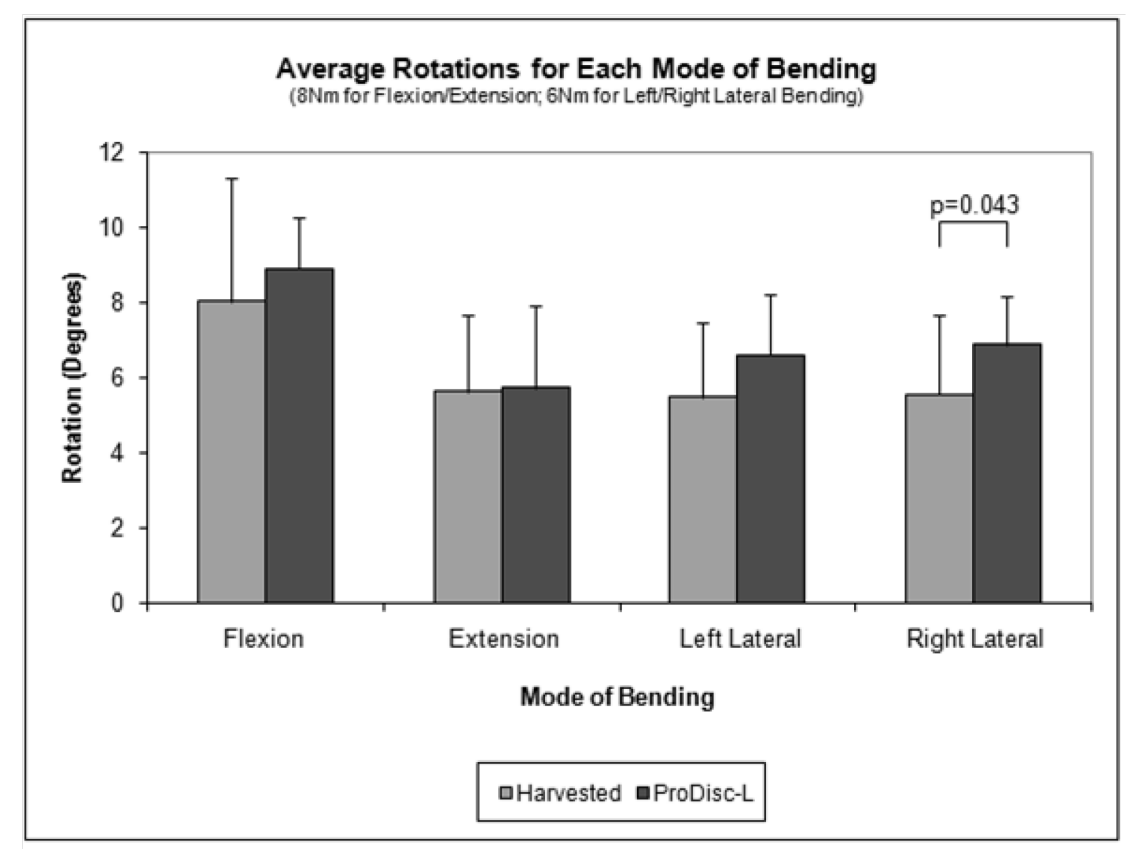

Figure 4-9 Comparing Ranges of Motion between Conditions. Comparison of ranges of motion for each condition during each mode of bending.

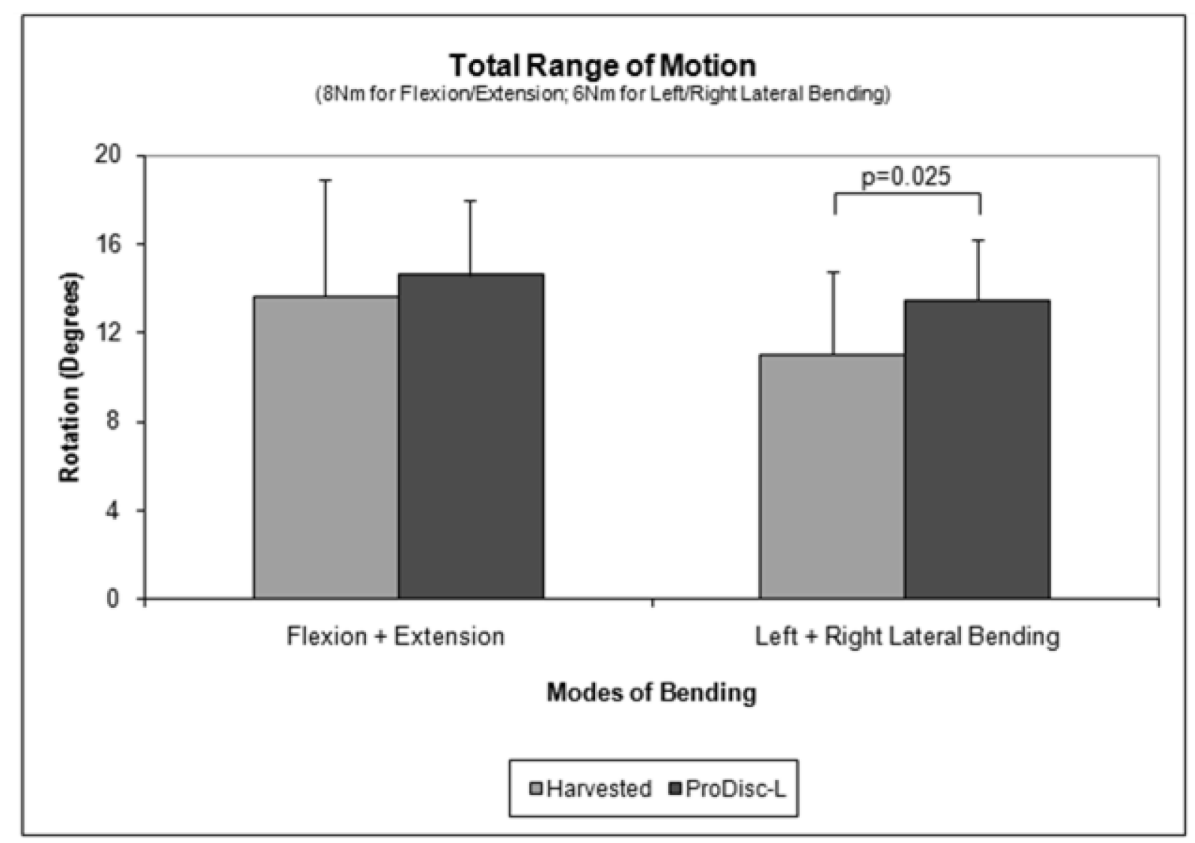

Figure 4-10 Comparing Total Ranges of Motion between Conditions. Comparison of total combined ranges of motion (flexion + extension and left + right lateral bending) for each condition. 
Table 4-1 Average Forces (N) along the Quasistatic Path.

\begin{tabular}{lcccc}
\hline Condition & Mode of Bending & Force & Mean & S.D. \\
\hline Harvested & Flexion & Shear Force & -0.34 & 1.08 \\
Harvested & Flexion & Axial Force & -0.49 & 1.13 \\
Harvested & Extension & Shear Force & -0.06 & 0.95 \\
Harvested & Extension & Axial Force & -0.54 & 1.12 \\
Harvested & Left Lateral & Shear Force & -0.46 & 0.77 \\
Harvested & Left Lateral & Axial Force & 0.23 & 1.10 \\
Harvested & Right Lateral & Shear Force & -0.28 & 0.87 \\
Harvested & Right Lateral & Axial Force & 0.10 & 1.18 \\
Implanted & Flexion & Shear Force & -0.12 & 1.29 \\
Implanted & Flexion & Axial Force & 0.15 & 1.72 \\
Implanted & Extension & Shear Force & -1.27 & 1.13 \\
Implanted & Extension & Axial Force & 0.05 & 1.94 \\
Implanted & Left Lateral & Shear Force & 0.11 & 1.25 \\
Implanted & Left Lateral & Axial Force & 0.51 & 1.97 \\
Implanted & Right Lateral & Shear Force & 0.34 & 1.20 \\
Implanted & Right Lateral & Axial Force & 1.00 & 1.39 \\
\hline
\end{tabular}




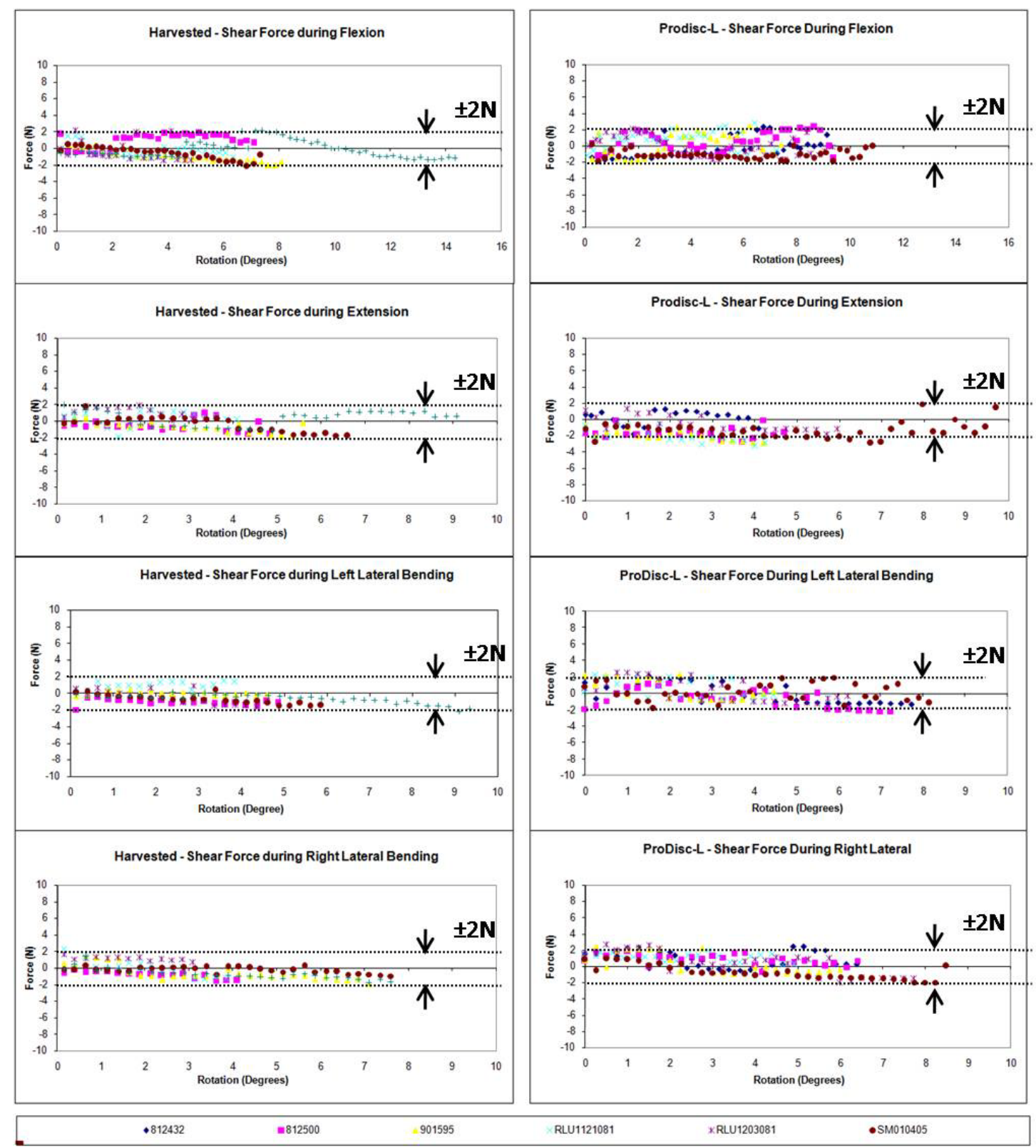

Figure 4-11 Specimen Shear Forces. Shear forces for each specimen during four modes of bending along quasistatic path. 

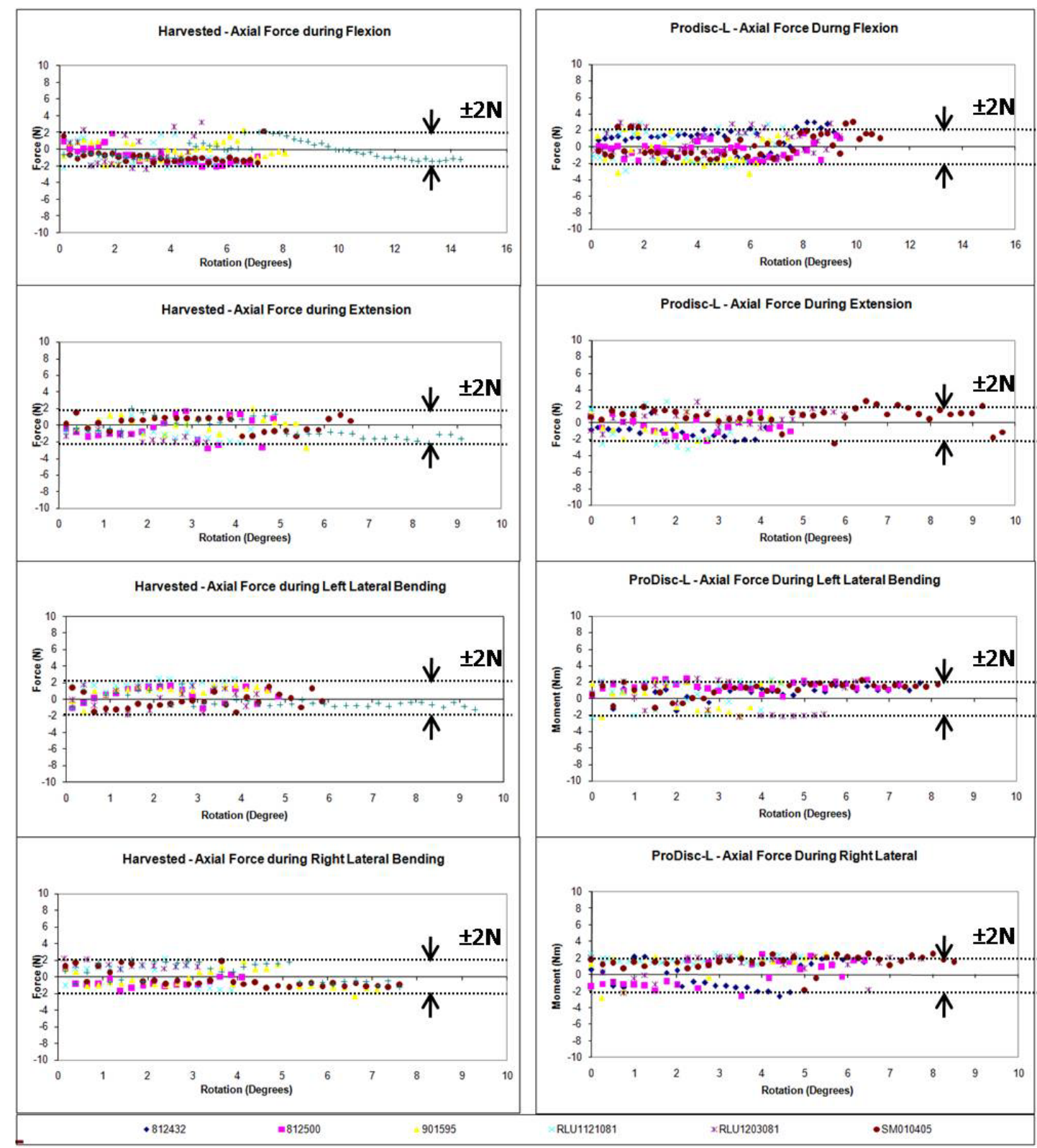

Figure 4-12 Specimen Axial Forces. Axial forces for each specimen during four modes of bending along the quasistatic path. 


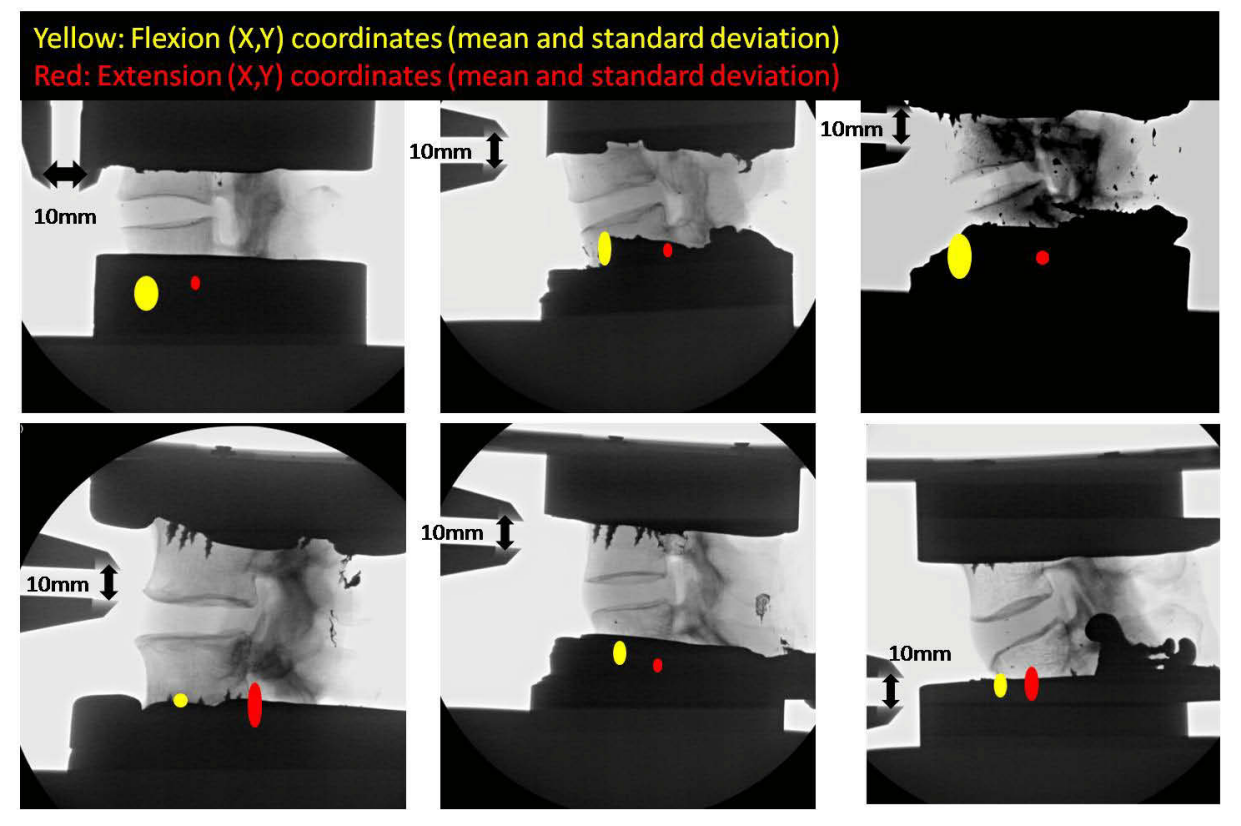

Figure 4-13 Calculated Harvested IAR Locations. The yellow and red zones represent the average and standard deviations of the flexion and extension locations, respectively. The calipers in each image are set to $10 \mathrm{~mm}$.

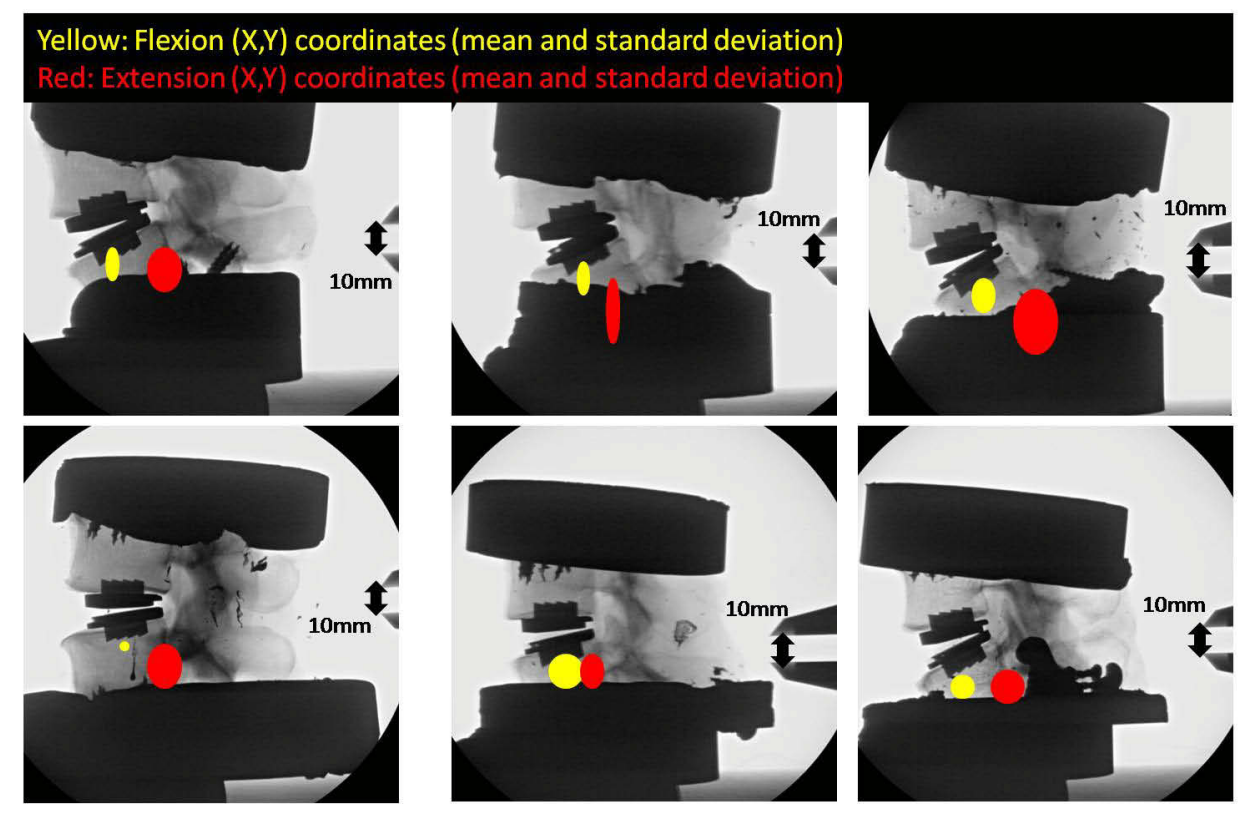

Figure 4-14 Calculated Implanted IAR Locations. The yellow and red zones represent the average and standard deviations of the flexion and extension locations, respectively. The calipers in each image are set to $10 \mathrm{~mm}$. 


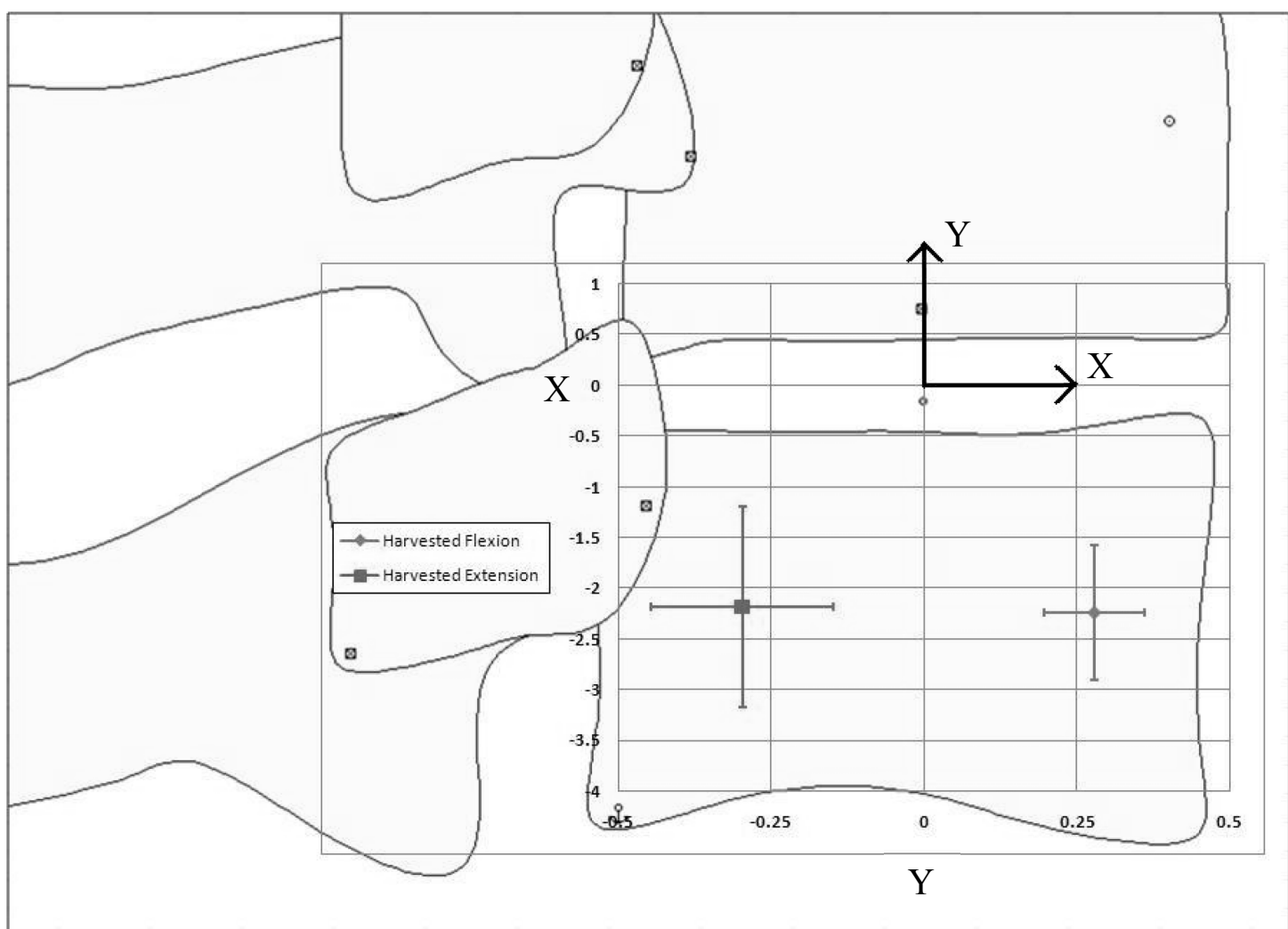

Figure 4-15 Normalized Harvested IAR Locations. Representative lumbar overlaid with a graph of the normalized harvested IAR locations (average with error bars). 1 unit on the $\mathrm{x}$-axis is equivalent to the sagittal length of the cranial vertebral body. 1 unit on the $\mathrm{y}$-axis is equivalent to harvested disc height. 


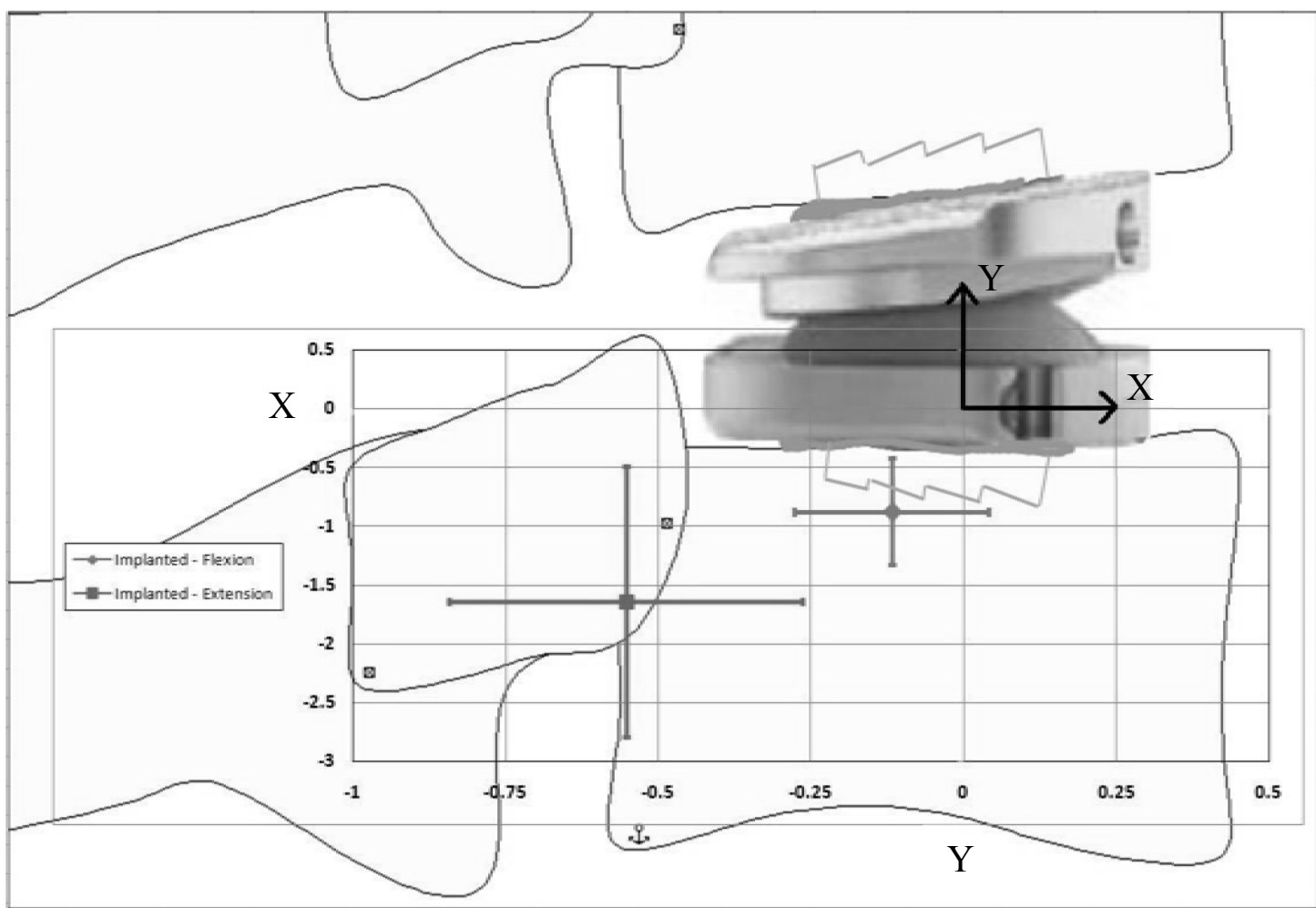

Figure 4-16 Normalized Implanted IAR Locations. Representative lumbar overlaid with a graph of the normalized implanted IAR locations (average with error bars). 1 unit on the $\mathrm{x}$-axis is equivalent to the sagittal length of the cranial vertebral body. 1 unit on the $\mathrm{y}$-axis is equivalent to harvested disc height. 
Table 4-2 Significant Differences among IAR Locations.

\begin{tabular}{ccc}
\hline Test & X-Values & Y-Values \\
\hline Harvested - Flexion vs. Extension & Yes $(\mathrm{p}<0.05)$ & No $(\mathrm{p}=0.193)$ \\
ProDisc-L - Flexion vs. Extension & Yes $(\mathrm{p}<0.05)$ & Yes $(\mathrm{p}<0.05)$ \\
Flexion - Harvested vs. ProDisc-L & Yes $(\mathrm{p}<0.05)$ & Yes $(\mathrm{p}<0.05)$ \\
Extension - Harvested vs. ProDisc-L & Yes $(\mathrm{p}<0.001)$ & Yes $(\mathrm{p}<0.05)$ \\
\hline
\end{tabular}

Differences between modes of bending and specimen conditions are shown. 


\subsection{Concluding Discussion}

The range of motion data indicate that for this form of testing the ProDisc-L implant imitates the biomechanical properties of the intact disc for flexion/extension bending. However, the lateral bending data indicate a significant difference between the right lateral bending data sets and the combined lateral bending data sets. This highlights the importance of the center of rotation of constrained devices. The increase in lateral bending is likely due to the constraining Prodisc-L implant disengaging the facet joints. Additionally, the fact that for individual bending modes only the right lateral bending showed a significant difference between conditions could indicate that the implant was improperly placed in the frontal plane. Because a constrained device is unable to compensate for small shifts in surgical positioning, careful placement is critical for ensuring a successful prosthesis.

The IAR shows a pronounced shift between flexion and extension for both the harvested and implanted conditions, which is in line with experimental observation. One would expect the IAR to lie in a posterior area during extension for the implanted specimens because it was observed that the cranial and caudal endplates actually detached throughout the bending motion. This is in contrast to flexion testing, where the IAR lied on average very near the center of the radius if the articulating polyethylene core. Under different testing conditions where shear forces aren't minimized, it is likely that additional shear forces would be put on the posterior structures, namely the facets.

One limitation of this study was the fact that only single-level specimens were tested. Testing full lumbar spines would allow for the study of adjacent level effects, such as motion compensation, a critical component to the long-term success of TDR components. Another limitation is the fact that no muscle vectors were simulated during testing. Simulating muscle vectors would allow for a much better representation of human movement and presumably better imitate in-vivo responses. Finally, axial rotation was not tested in this research. 


\section{Chapter 5 - Conclusions and Limitations}

The initial motivation for this study was to develop a pure moment testing protocol that was significantly more accurate than common testing platforms in the published literature. The University of Tennessee Health Science Center Spine Robot has extremely high resolution translational axes, which we believed would be suitable for a pure moment methodology. As shown in Chapter 3, off-axis loading was reduced almost completely within the protocol, and was not a significant source of error. The cable-and-pulley setup favored by many labs is incapable of producing pure rotational loading free of off-axis forces acting on the specimen. The range of motion and flexibility data derived during testing showed similar results to other in-vitro and in-vivo testing, indicating that the designed protocol can be suitably compared to current methods.

The second motivation for the study was the calculation of the IAR for the spinal segments. The resolution of the Spine Robot's axes give positional data that is more precise than data for other in-vitro methods or radiographic data for in-vivo methods. Testing showed a clear, significant A-P separation of the IAR locations for segments under all conditions. As mentioned previously, this was not a surprise since less precise measurements would naturally tend to the average of these separate points, which is what is seen in other published data. It is important to note that although shifts in the IAR occurred, this did not excessively alter the biomechanics of the instrumented segment compared to the harvested state for this passive pure moment testing.

The proximity of the calculated IAR of the implanted specimen during flexion to the COR of the implant itself highlights the accuracy of the methodology. The shift of the IAR of implanted specimen under extension testing indicates a physical separation of the joint, a behavior that was observed during testing. Although tests were only performed under passive conditions, a clear separation of the joint might indicate an instability resulting from the device's design and implantation procedure. In the case of the ProDisc-L implant, this behavior might also lead to additional shear stresses on the polyethylene inlay within the prosthesis. IAR tracking is an important tool for understanding the kinematic characteristics of both the intact spine and those that have been surgically altered.

Some limitations exist in this research. First, the testing only included singlelevel MSUs, and only included L4-L5 segments. Testing of multiple levels would allow for study of motion compensation at adjacent levels and implants at multiple levels. Additionally, the L5-S1 segment is important to research as it accounts for a large amount of lumbar rotation. ${ }^{60}$ Next, because there was no simulation of a muscle vector within the MSU, the testing cannot be said to show the dynamics of the joint. However, because the testing conditions represent a passive condition for the joint the kinematic behavior of the joint was observed. Testing only included sagittal and lateral bending, excluding axial rotation. As axial rotation is one of the three principal rotations of the spine, it is a necessary component in quantifying spinal motion. Finally, the placement of 
the implants was determined by a spine surgeon and was not a parameter that was controlled for. The placement of the implant can affect motion response and mechanics in patients and laboratory settings. ${ }^{4,29}$ 


\section{Chapter 6 - Recommendations for Future Work}

One recommendation for future work would be to complete the pure moment testing protocol on a full lumbar specimen. With an entire lumbar column, researchers would be able to observe motion compensation at adjacent levels and test implanted conditions with devices on multiple levels. Additionally, full lumbar testing would be much more suitable for incorporating a fusion model as a test condition, since a singlelevel fusion model would ideally yield no motion. A second recommendation would be to incorporate coupled pure moment testing into the protocol. The Spine Robot has the capacity to simultaneously apply sagittal bending and axial rotation or lateral bending with axial rotation. Incorporating coupled motions into the protocol would not require significant modification to the current program code.

A compressive force to simulate muscle response could be implemented to represent a physiologically active simulation as opposed to the passive simulation in the current study. This compressive force could be created with the current actuators themselves, and would not require any additional external equipment. The positional control of the Spine Robot would allow for more accurate placement of the simulated muscle vectors with the potential to study individual or multiple muscles. Another potential addition would be a physiological shear force to study its affect on motion and IAR location. Conditions of extreme shear could be detrimental to the stability of TDR devices, and several studies have hypothesized that it might play a role in the dislocation of the polyethylene inlay of the Prodisc-L. ${ }^{29,40}$

Finally, it would be beneficial to create a pure moment protocol that could create the loading conditions in real-time as opposed to quasi-static. We believe that the Spine Robot is capable of producing a pure moment response in real-time, but is not possible with current knowledge. This study would be remiss without mentioning how much potential the Spine Robot has for spine biomechanics research. Hopefully the full capabilities of this device will be realized in the near future. 


\section{List of References}

1. Abumi K, Panjabi MM, Kramer KM, et al. Biomechanical evaluation of lumbar spine stability after graded facetectomies. Spine 1990;15:1142-1147.

2. An HS, Simpson JM, eds. Surgery of the Cervical Spine. Baltimore: Williams \& Wilkins Co., 1994.

3. Bao QB, McCullen GM, Higham PA, et al. The artificial disc; theory, design, and materials. Biomaterials 1996;17:1157-1167.

4. Bendo JA MD, Quirno M MD, Errico T MD, et al. A comparison of two retroperitoneal surgical approaches for total disc arthroplasty of the lumbar spine. Spine 2008;33:205-209.

5. Benzel EC, ed. Spine Surgery: Techniques, Complications, Avoidance, and Management. 2nd ed. Philadelphia: Elsevier Churchill Livingstine, 2005.

6. Bonin H. In-vitro analysis of the instantaneous center of rotation in a human cervical spine model using a spine robot. Master's Thesis. The University of Tennessee Health Science Center, 2006.

7. Butler J, Lewis R, Shier D, et al. Hole's Human Anatomy \& Physiology. 9th ed. Boston: McGraw Hill, 2002.

8. Clark CR, ed. The Cervical Spine. 3rd ed. Philadelphia: Lippincott-Raven Pub., 1998.

9. Crawford MJB, Doherty BJP, Dreyzin VM, et al. Kinematic evaluation of lumbar fusion techniques. Spine 1996;21:676-684.

10. Crawford NR, Brantley AG, Dickman CA, et al. An apparatus for applying pure unconstraining moments to spines in vitro. Spine 1995;20:2097-2100.

11. Crawford NR. Letter to the Editor. Clinical Biomechanics 2007;22:861-862.

12. Crisco JJ I, Chen X, Panjabi MM, et al. Optimal marker placement for calculating instantaneous center of rotation. Journal of Biomechanics 1994;27:1183-1187.

13. Cunningham BW, Gordon JD, Dmitriev AE, et al. Biomechanical evaluation of total disc replacement artroplasty: an in-vitro human cadaveric model. Spine 2003;28:S110-S117.

14. Dabnicki P, Lauder M, Aritan S, et al. Accuracy evaluation of an on-line kinematic system via dynamic tests. Journal of Medical Engineering Technology 1997;21:5366. 
15. Deguchi M, Cheng BC, Sato K, et al. Biomechanical evaluation of translaminar facet joint fixation. A comparative study of poly-L-lactide pins, screws, and pedicle fixation. Spine 1998;23:1307-1312.

16. DiAngelo DJ, Scifert J, Kitchel S, et al. Bioabsorbable anterior lumbar plate fixation in conjunction with cage-assisted anterior interbody fusion. Journal of Neurosurgery 2002;4:447-455.

17. DiAngelo DJ, Foley KT. An improved biomechanical testing protocol for evaluation multilevel cervial intrumentation in a human cadaveric corpectomy model. Spinal Implants: Are We Evaluating Them Properly? 2003;155-172.

18. DiAngelo DJ, Robertson JT, Metcalf NH, et al. Biomechanical testing of an artificial cervical joint and an anterior cervical plate. Journal of Spinal Disorder Technology 2003;16:314-323.

19. Dickey JP, Gillespie KA. Representation of passive spinal element contributions to in vitro flexion-extension using a polynomial model: illustration using the porcine lumbar spine. Journal of Biomechanics 2003;36:883-888.

20. Dvorak J, Panjabi MM, Chang DG, et al. Functional radiographic diagnosis of the lumbar spine: flexion/extension and lateral bending. Spine 1989;16:562-571.

21. Eguizabal J, Tufaga M, Scheer JK, et al. Pure Moment testing for spinal biomechanics applications: fixed versus sliding ring cable-driven test designs. Journal of Biomechanics 2010; in press.

22. Froning EC, Frohman B. Motion of the lumbosacral spine after laminectomy and spinal fusion: Correlation of motion with the result. Journal of Bone and Joint Surgery - American Volume 1968;50-A:897-918.

23. Frymoyer JW, ed. The Adult Spine: Principles and Practice. 2nd ed. Philadelphia: Lippincott-Raven, 1997.

24. Fuchs PD, Lindsey DP, Hsu KY, et al. The use of an interspinous implant in conjunction with a graded facetectomy procedure. Spine 2005;30:1266-1272.

25. Goel VK, Clark CR, McGowan D, et al. An in-vitro study of kinematics of the normal, injured and stabilized cervical spine. Journal of Biomechanics $1984 ; 17: 363-376$.

26. Goel VK, Goyal S, Clark CR, et al. Kinematics of the whole lumbar spine. Effect of discectomy. Spine 1985; 10:543-554.

27. Goel VK, Wilder DG, Pope MH, et al. Biomechanical testing of the spine. Loadcontrolled versus displacement-controlled analysis. Spine 1995;20:2354-2357. 
28. Herkowitz HN, Garfin SR, Balderston RA, et al. Rothman-Simeone: The Spine. 4th ed. Philadelphia: W.B. Saunders Co., 1999.

29. Huang RC, Girardi FP, Cammisa Jr.FP, et al. The implications of constraint in lumbar total disc replacement. Journal of Spinal Disorders and Techniques 2010;16:412-417.

30. Humzah MD, Soames RW. Human intervertebral disc: structure and function. The Anatomical Record 1988;220:337-356.

31. Ianuzzi A, Zambrano I, Tataria $\mathrm{J}$ et al. Biomechanical evaluation of surgical constructs for stabilization of cervical teardrop fractures. The Spine Journal 2006;6:514-523.

32. Johnson WM, Rapoff AJ. Three degree of freedom biomechanical testing system. Proceedings of the American Society of Mechanical Engineers, 2001.

33. Jones GA, Kayanja M, Milks R, et al. Biomechanical characteristics of hybrid hook-screw constructs in short-segment thoracic fixation. Spine 2008;33:173-177.

34. Kanayama M, Cunningham BW, Weis JC, et al. The effects of rigid spinal instrumentation and solid bony fusion on spinal kinematics. A posterolateral spinal arthrodesis model. Spine 1998;23:767-773.

35. Kelly BP. A multiaxis programmable spine robot for the study of multibody spinal biomechanics using real-time hybrid force and displacement control strategies. $\mathrm{PhD}$ Dissertation. The University of Tennessee Health Science Center, 2005.

36. Kunz DN, McCabe RP, Zdeblick TA, et al. A multi-degree of freedom system for biomechaincal testing. Journal of Biomechanical Engineering 1994;116:371-373.

37. Lawrence JS. Disc degeneration. Its frequency and relationship to symptoms. Annals of the Rheumatic Diseases 1969;28:121-138.

38. Manchikanti L, Manchikanti K, Cash K, et al. Age-related prevelance of facet-joint involvement in chronic neck and low back pain. Pain Physician 2008;11:67-75.

39. Martin MD, Boxell CM, Malone DG. Pathophysiology of lumbar disc degeneration: a review of the literature. Neurosurgical Focus 2002;13:1-6.

40. Mathew P, Blackman M, Redla S, et al. Bilateral pedicle fractures following anterior dislocation of the polyethylene inlay of a ProDisc artificial disc replacement. Spine 2005;30:E311-E314.

41. Ogston NG, King GJ, Gertzbein SD, et al. Centrode Pattersn in the lumbar spine: baseline studies in normal subjects. Spine 1986;11:591-595. 
42. Panjabi MM. Biomechanical evaluation of spinal fixation devices: I. A conceptual framework. Spine 1988;13:1129-1134.

43. Panjabi MM. The stabilizing system of the spine. Part II: Neutral Zone and Instability Hypothesis. Journal of Spinal Disorders 1992;5:390-396.

44. Panjabi MM, Oxland TR, Yamamoto I, et al. Mechanical behavior of the human lumbar and lumbosacral spine as shown by three-dimensional load-displacement curves. Journal of Bone and Joint Surgery - American Volume 1994;76:413-424.

45. Panjabi MM, Lydon C, Vasavada A, et al. On the understanding of clinical instability. Spine 1994;19:2642-2650.

46. Panjabi MM. Hybrid multidirectional test method to evaluate spinal adjacent-level effects. Clinical Biomechanics 2007;22:257-265.

47. Panjabi MM, Henderson G, Abjornson C, et al. Multidirectional testing one and two-level Prodisc-L versus simulated fusions. Spine 2007;32:1311-1319.

48. Patwardhan AG, Havey RM, Meade KP, et al. A follower load increases the loadcarrying capacity of the lumbar spine in compression. Spine 1999;24:1003-1009.

49. Pearcy MJ, Tibrewal S. Lumbar intervertebral disc and ligament deformation measured in-vivo. Clinical Orthopedics 1984;191:281-286.

50. Pearcy MJ, Bogduk N. Instantaneous axes of rotation of the lumbar intervertebral joints. Spine 1988;13:1033-1041.

51. Porterfield JA, DeRosa C. Mechanical Neck Pain: Perspectives in Functional Anatomy. 1st ed. Philadelphia: W.B. Saunders Co., 1995.

52. Roberts S. Disc Morphology in health and disease. Biochemical Society Transactions 2002;30:864-869.

53. Sakamaki T, Katoh S, Sairyo K. Normal and spondylolytic pediatric spine movement with reference to instantaneous axis of rotation. Spine 2002;27:141-145.

54. Schneider G, Pearcy MJ, Bogduk N. Abnormal motion in spondylolytic spondylolisthesis. Spine 2005;30:1159-1164.

55. Schultz AB, Wareick DN, Berkson $\mathrm{MH}$, et al. Mechanical properties of human lumbar spine motion segments - responses in flexion, extension, lateral bending and torsion. Journal of Biomechanical Engineering 1979;101:46-52.

56. Seligman JV, Gertzbein SD, Tile M, et al. Computer analysis of spinal segment motion in degenerative disc disease with and without axial loading (Volvo award in basic science). Spine 1984;9:566-573. 
57. Sobajima S, Kim JS, Gilbertson LG, et al. Gene therapy for degenerative disc disease. Gene Therapy 2004;11:390-401.

58. Spenciner D, Greene D, Paiva J, et al. The multidirectional bending properties of the human lumar intervertebral disc. The Spine Journal 2006;6:248-257.

59. Tzermiadianos MN, Mekhail A, Voronov LI, et al. Enhancing the stability of anterior lumbar interbody fusion: a biomechanical comparison of anterior plate versus posterior transpedicular instrumentation. Spine 2008;33:E38-E43.

60. White AA, Panjabi MM. Clinical Biomechanics of the Spine. 2nd ed. Philadelphia: J.B. Lippincott Co., 1990.

61. Wilke HJ, Claes L, Schmitt H, et al. A universal spine tester for in-vitro experiments with muscle force simulator. European Spine Journal 1994;3:91-97.

62. Wilke HJ, Wenger K, Claes L. Testing criteria for spinal implants: recommendations for the standardization of in vitro stability testing of spinal implants. European Spine Journal 1998;7:148-154.

63. Wilke HJ, Rohlmann A, Neller S, et al. Is it possible to simulate physiological loading conditions by applying pure moments? A comparison of in vivo and in vitro load components in an internal fixator. Spine 2001;26:636-642.

64. Winter DA. Biomechanics of Human Movement. New York: John Wiley \& Sons, 1979.

65. Yamamoto I, Panjabi MM, Crisco T., et al. Three-dimensional movements of the whole lumbar spine and lumbosacral joint. Spine 1989;14:1256-1260.

66. Yoshioka T, Tsuji H, Hirano N, et al. Motion characteristics of the normal lumbar spine in young adults: instantaneous axis of rotation and vertebral center motion analyses. Journal of Spinal Disorders 1990;3:103-113.

67. Zigler J MD, Delamarter R MD, Spivak JM MD, et al. Results of the prospective, randomized, multicenter food and drug administration investigational device exemption study of the prodisc-1 total disc replacement versus circumferential fusion for the treatment of 1-level degenerative disc disease. Spine 2007;32:11551162 .

68. Zufelt N. A kinematics-based testing protocol to study the mechanics of the human lumbar spine. Master's Thesis. The University of Tennessee Health Science Center, 2008. 
Kyle David Fraysur was born on March 10, 1984 in Columbus, Mississippi. He spent his formative years in Jackson, Mississippi, where he attended Forest Hill High School. It was at Forest Hill that he met Elizabeth, the woman that would become his future wife. Upon graduation, Kyle moved to Starkville, Mississippi to attend Mississippi State University, pursuing a degree in Biological Engineering. After graduating with a Bachelor's Degree in 2006, he decided to take a year off to work while Elizabeth completed her undergraduate education. On May 19, 2007, they were married.

In July, Kyle and Elizabeth moved to Memphis, Tennessee to allow him to pursue a graduate degree in Biomedical Engineering. He joined the Joint-Implant Biomechanics Laboratory under the tutelage of Dr. Denis DiAngelo. While in the lab, he completed multiple projects in spinal biomechanics, had the opportunity to meet and collaborate with people from industry and clinicians, and made some good friends. In September of 2009, Elizabeth gave birth to a baby boy named Greyson David. In September of 2009, Kyle accepted a position as an Engineer at Cagenix, Inc., a dental products company in Memphis. 